\title{
Efficient Modelling of Wind Turbine Foundations
}

\author{
Lars Andersen and Johan Clausen \\ Aalborg University, Department of Civil Engineering
}

Denmark

\section{Introduction}

Recently, wind turbines have increased significantly in size, and optimization has led to very slender and flexible structures. Hence, the Eigenfrequencies of the structure are close to the excitation frequencies related to environmental loads from wind and waves. To obtain a reliable estimate of the fatigue life of a wind turbine, the dynamic response of the structure must be analysed. For this purpose, aeroelastic codes have been developed. Existing codes, e.g. FLEX by Øye (1996), HAWC by Larsen \& Hansen (2004) and FAST by Jonkman \& Buhl (2005), have about 30 degrees of freedom for the structure including tower, nacelle, hub and rotor; but they do not account for dynamic soil-structure interaction. Thus, the forces on the structure may be over or underestimated, and the natural frequencies may be determined inaccurately.
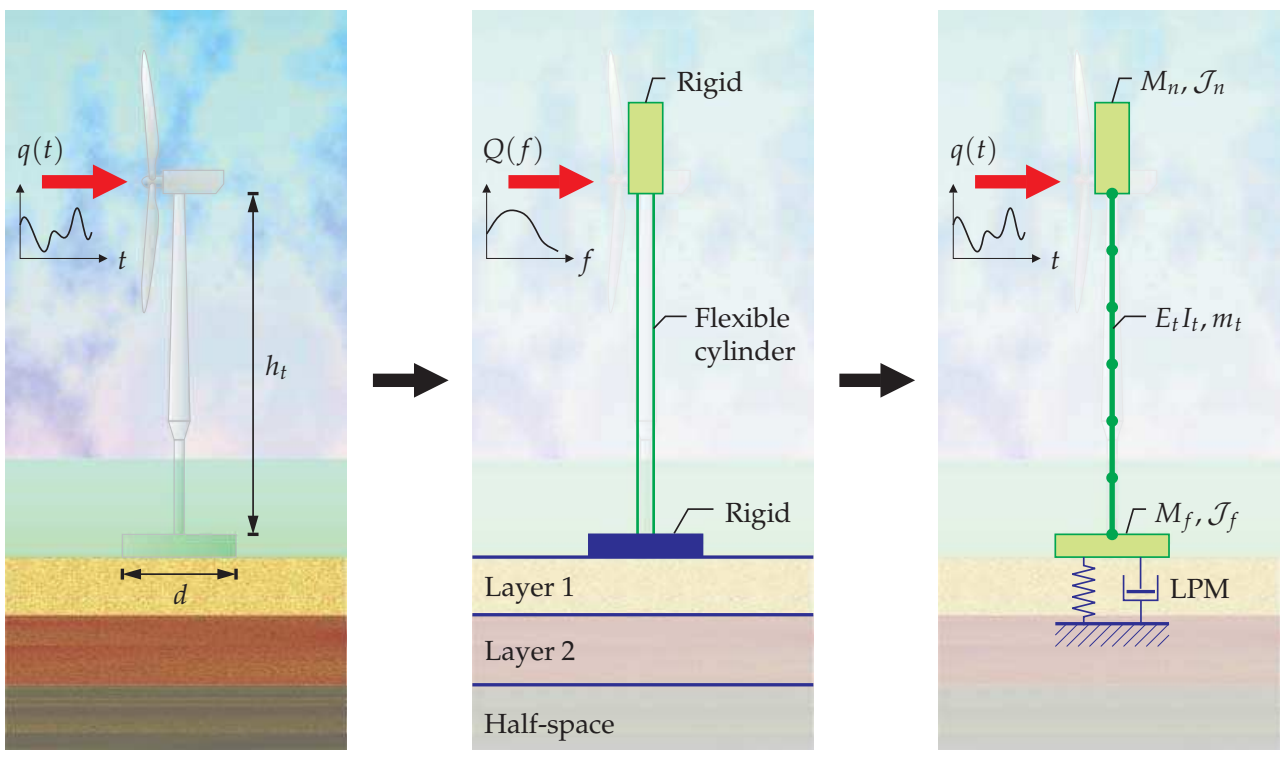

Fig. 1. From prototype to computational model: Wind turbine on a footing over a soil stratum (left); rigorous model of the layered half-space (centre); lumped-parameter model of the soil and foundation coupled with finite-element model of the structure (right). 
Andersen \& Clausen (2008) concluded that soil stratification has a significant impact on the dynamic stiffness, or impedance, of surface footings-even at the very low frequencies relevant to the first few modes of vibration of a wind turbine. Liingaard et al. (2007) employed a coupled finite-element/boundary-element model for the analysis of a flexible bucket foundation, finding a similar variation of the dynamic stiffness in the frequency range relevant for wind turbines. This illustrated the necessity of implementing a model of the turbine foundation into the aeroelasic codes that are utilized for design and analysis of the structure. However, since computation speed is of paramount importance, the model of the foundation should only add few degrees of freedom to the model of the structure. As proposed by Andersen (2010) and illustrated in Fig. 1, this may be achieved by fitting a lumped-parameter model (LPM) to the results of a rigorous analysis, following the concepts outline by Wolf (1994).

This chapter outlines the methodology for calibration and implementation of an LPM of a wind turbine foundation. Firstly, the formulation of rigorous computational models of foundations is discussed with emphasis on rigid footings, i.e. monolithic gravity-based foundations. A brief introduction to other types of foundations is given with focus on their dynamic stiffness properties. Secondly, Sections 2 and 3 provide an in-depth description of an efficient method for the evaluation of the dynamics stiffness of surface footings of arbitrary shapes. Thirdly, in Section 4 the concept of consistent lumped-parameter models is presented and the formulation of a fitting algorithm is discussed. Finally, Section 5 includes a number of example results that illustrate the performance of lumped-parameter models.

\subsection{Types of foundations and their properties}

The gravity footing is the only logical choice of foundation for land-based wind turbines on residual soils, whereas a direct anchoring may be applied on intact rock. However, for offshore wind turbines a greater variety of possibilities exist. As illustrated in Fig. 2, when the turbines are taken to greater water depths, the gravity footing may be replaced by a monopile, a bucket foundation or a jacket structure. Another alternative is the tripod which, like the jacket structure, can be placed on piles, gravity footings or spud cans (suction anchors). The latter case was studied by Senders (2005). In any case, the choice of foundation type is site dependent and strongly influenced by the soil properties and the environmental conditions, i.e. wind, waves, current and ice. Especially, current may involve sediment transport and scour on sandy and silty seabeds, which may lead to the necessity of scour protection around foundations with a large diameter or width.

Regarding the design of a wind turbine foundation, three limit states must be analysed in accordance with most codes of practice, e.g. the Eurocodes. For offshore foundations, design is usually based on the design guidelines provided by the API (2000) or DNV (2001). Firstly, the strength and stability of the foundation and subsoil must be high enough to support the structure in the ultimate limit state (ULS). Secondly, the stiffness of the foundation should ensure that the displacements of the structure are below a threshold value in the serviceability limit state (SLS). Finally, the wind turbine must be analysed regarding failure in the fatigue limit state (FLS), and this turns out to be critical for large modern offshore wind turbines.

The ULS is typically design giving for the foundations of smaller, land-based wind turbines. In the SLS and FLS the turbine may be regarded as fully fixed at the base, leading to a great simplification of the dynamic system to be analysed. However, as the size of the turbine increases, soil-structure interaction becomes stronger and due to the high flexibility of the structure, the first Eigenfrequencies are typically below $0.3 \mathrm{~Hz}$. 


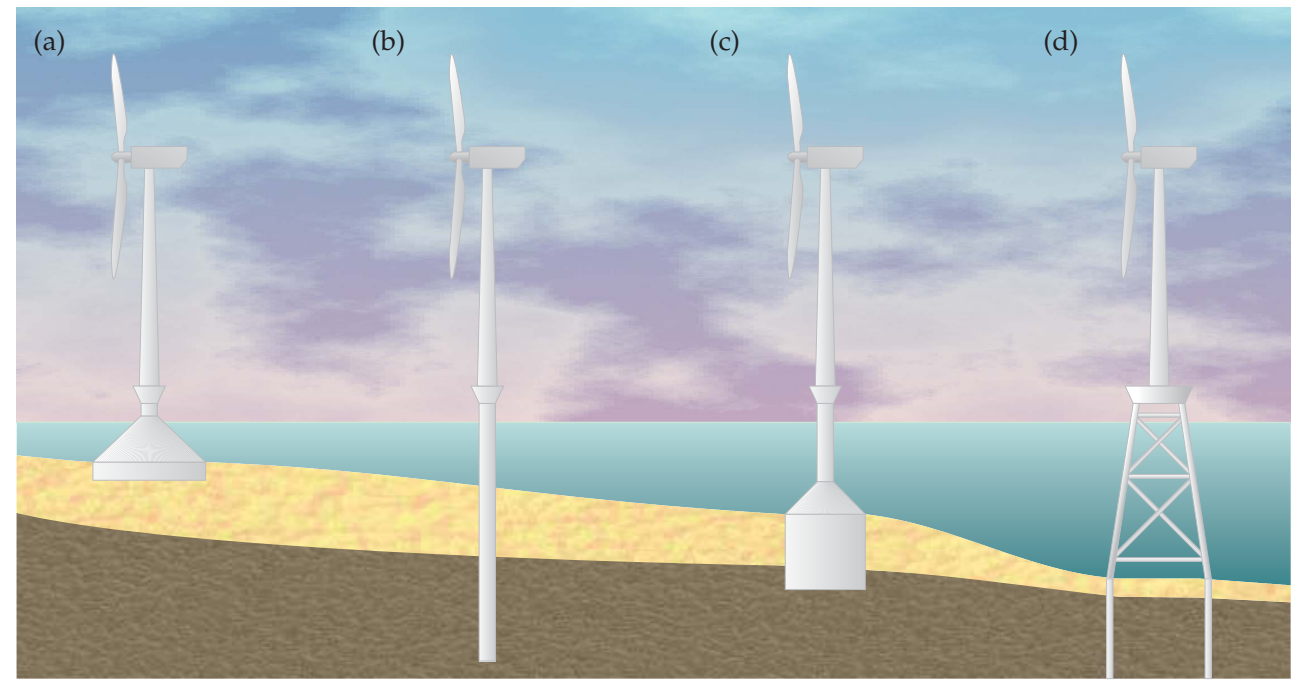

Fig. 2. Different types of wind turbine foundations used offshore a various water depths:

(a) gravity foundation; (b) monopile foundation; (c) monopod bucket foundation and

(d) jacket foundaiton.

An improper design may cause resonance due to the excitation from wind and waves, leading to immature failure in the FLS. An accurate prediction of the fatigue life span of a wind turbine requires a precise estimate of the Eigenfrequencies. This in turn necessitates an adequate model for the dynamic stiffness of the foundation and subsoil. The formulation of such models is the focus of such models. The reader is referred to standard text books on geotechnical engineering for further reading about static behaviour of foundations.

\subsection{Computational models of foundations for wind turbines}

Several methods can be used to evaluate the dynamic stiffness of footings resting on the surface of the ground or embedded within the soil. Examples include analytic, semi-analytic or semi-empirical methods as proposed by Luco \& Westmann (1971), Luco (1976), Krenk \& Schmidt (1981), Wong \& Luco (1985), Mita \& Luco (1989), Wolf (1994) and Vrettos (1999) as well as Andersen \& Clausen (2008). Especially, torsional motion of footings was studied by Novak \& Sachs (1973) and Veletsos \& Damodaran Nair (1974) as well as Avilés \& Pérez-Rocha (1996). Rocking and horizontal sliding motion of footings was analysed by Veletsos \& Wei (1971) and Ahmad \& Rupani (1999) as well as Bu \& Lin (1999). Alternatively, numerical analysis may be conducted using the finite-element method and the boundary-element method. See, for example, the work by Emperador \& Domínguez (1989) and Liingaard et al. (2007).

For monopiles, analyses are usually performed by means of the Winkler approach in which the pile is continuously supported by springs. The nonlinear soil stiffness in the axial direction along the shaft is described by $t-z$ curves, whereas the horizontal soil resistance along the shaft is provided by $p-y$ curves. Here, $t$ and $p$ is the resulting force per unit length in the vertical and horizontal directions, respectively, whereas $z$ and $y$ are the corresponding displacements. For a pile loaded vertically in compression, a similar model can be formulated for the tip 
resistance. More information about these methods can be found in the design guidelines by API (2000) and DNV (2001).

Following this approach, El Naggar \& Novak (1994a;b) formulated a model for vertical dynamic loading of pile foundations. Further studies regarding the axial response were conducted by Asgarian et al. (2008), who studied pile-soil interaction for an offshore jacket, and Manna \& Baidya (2010), who compared computational and experimental results. In a similar manner, El Naggar \& Novak $(1995 ; 1996)$ studied monopiles subject to horizontal dynamic excitation. More work along this line is attributed to El Naggar \& Bentley (2000), who formulated $p-y$ curves for dynamic pile-soil interaction, and Kong et al. (2006), who presented a simplified method including the effect of separation between the pile and the soil. A further development of Winkler models for nonlinear dynamic soil behaviour was conducted by Allotey \& El Naggar (2008). Alternatively, the performance of mononpiles under cyclic lateral loading was studied by Achmus et al. (2009) using a finite-element model. Gerolymos \& Gazetas (2006a;b;c) developed a Winkler model for static and dynamic analysis of caisson foundations fully embedded in linear or nonlinear soil. Further research regarding the formulation of simple models for dynamic response of bucket foundations was carried out by Varun et al. (2009). The concept of the monopod bucket foundation has been described by Houlsby et al. $(2005 ; 2006)$ as well as Ibsen (2008). Dynamic analysis of such foundations were performed by Liingaard et al. $(2007 ; 2005)$ and Liingaard $(2006)$ as well as Andersen et al. (2009). The latter work will be further described by the end of this chapter.

\section{Semi-analytic model of a layered ground}

This section provides a thorough explanation of a semi-analytical model that may be applied to evaluate the response of a layered, or stratified, ground. The derivation follows the original work by Andersen \& Clausen (2008). The fundamental assumption is that the ground may be analysed as a horizontally layered half-space with each soil layer consisting of a homogeneous linear viscoelastic material. In Section 3 the model of the ground will be used as a basis for the development of a numerical method providing the dynamic stiffness of a foundation over a stratum. Finally, in Section 5 this method will be applied to the analysis of gravity-based foundations for offshore wind turbines.

\subsection{Response of a layered half-space}

The surface displacement in time domain and in Cartesian space is denoted $u_{i}^{10}\left(x_{1}, x_{2}, t\right)=$ $u_{i}\left(x_{1}, x_{2}, 0, t\right)$. Likewise the surface traction, or the load on the free surface, will be denoted $p_{i}^{10}\left(x_{1}, x_{2}, t\right)=p_{i}\left(x_{1}, x_{2}, 0, t\right)$. An explanation of the double superscript 10 is given in the next subsection. Here it is just noted that superscript 10 refers to the top of the half-space.

Further, let $g_{i j}\left(x_{1}-y_{1}, x_{2}-y_{2}, t-\tau\right)$ be the Green's function relating the displacement at the observation point $\left(x_{1}, x_{2}, 0\right)$ to the traction applied at the source point $\left(y_{1}, y_{2}, 0\right)$. Both points are situated on the surface of a stratified half-space with horizontal interfaces. The total displacement at the point $\left(x_{1}, x_{2}, 0\right)$ on the surface of the half-space is then found as

$$
u_{i}^{10}\left(x_{1}, x_{2}, t\right)=\int_{-\infty}^{t} \int_{-\infty}^{\infty} \int_{-\infty}^{\infty} g_{i j}\left(x_{1}-y_{1}, x_{2}-y_{2}, t-\tau\right) p_{j}^{10}\left(y_{1}, y_{2}, \tau\right) \mathrm{d} y_{1} \mathrm{~d} y_{2} d \tau .
$$

The displacement at any point on the surface of the half-space and at any instant of time may be evaluated by means of Eq. (1). However, this requires the existence of the Green's function $g_{i j}\left(x_{1}-y_{1}, x_{2}-y_{2}, t-\tau\right)$, which may be interpreted as the dynamic flexibility. Unfortunately, 
a closed-form solution cannot be established for a layered half-space, and in practice the temporal-spatial solution expressed by Eq. (1) is inapplicable.

Assuming that the response of the stratum is linear, the analysis may be carried out in the frequency domain. The Fourier transformation of the surface displacements with respect to time is defined as

$$
U_{i}^{10}\left(x_{1}, x_{2}, \omega\right)=\int_{-\infty}^{\infty} u_{i}^{10}\left(x_{1}, x_{2}, t\right) \mathrm{e}^{-\mathrm{i} \omega t} \mathrm{~d} t
$$

with the inverse Fourier transformation given as

$$
u_{i}^{10}\left(x_{1}, x_{2}, t\right)=\frac{1}{2 \pi} \int_{-\infty}^{\infty} U_{i}^{10}\left(x_{1}, x_{2}, \omega\right) \mathrm{e}^{\mathrm{i} \omega t} \mathrm{~d} \omega .
$$

Likewise, a relationship can be established between the surface load $p_{i}^{10}\left(x_{1}, x_{2}, t\right)$ and its Fourier transform $P_{i}^{10}\left(x_{1}, x_{2}, \omega\right)$, and similar transformation rules apply to the Green's function, i.e. between $g_{i j}\left(x_{1}-y_{1}, x_{2}-y_{2}, t-\tau\right)$ and $G_{i j}\left(x_{1}-y_{1}, x_{2}-y_{2}, \omega\right)$. It then follows that

$$
U_{i}^{10}\left(x_{1}, x_{2}, \omega\right)=\int_{-\infty}^{\infty} \int_{-\infty}^{\infty} G_{i j}\left(x_{1}-y_{1}, x_{2}-y_{2}, \omega\right) P_{j}^{10}\left(y_{1}, y_{2}, \omega\right) \mathrm{d} y_{1} \mathrm{~d} y_{2},
$$

reducing the problem to a purely spatial convolution.

Further, assuming that all interfaces are horizontal, a transformation is carried out from the Cartesian space domain description into a horizontal wavenumber domain. This is done by a double Fourier transformation in the form

$$
\bar{U}_{i}^{10}\left(k_{1}, k_{2}, \omega\right)=\int_{-\infty}^{\infty} \int_{-\infty}^{\infty} U_{i}^{10}\left(x_{1}, x_{2}, \omega\right) \mathrm{e}^{-\mathrm{i}\left(k_{1} x_{1}+k_{2} x_{2}\right)} \mathrm{d} x_{1} \mathrm{~d} x_{2}
$$

where the double inverse Fourier transformation is defined by

$$
U_{i}^{10}\left(x_{1}, x_{2}, \omega\right)=\frac{1}{4 \pi^{2}} \int_{-\infty}^{\infty} \int_{-\infty}^{\infty} \bar{U}_{i}^{10}\left(k_{1}, k_{2}, \omega\right) \mathrm{e}^{\mathrm{i}\left(k_{1} x_{1}+k_{2} x_{2}\right)} \mathrm{d} k_{1} \mathrm{~d} k_{2} .
$$

By a similar transformation of the surface traction and the Green's function, Eq. (4) finally achieves the form

$$
\bar{U}_{i}^{10}\left(k_{1}, k_{2}, \omega\right)=\bar{G}_{i j}\left(k_{1}, k_{2}, \omega\right) \bar{P}_{j}^{10}\left(k_{1}, k_{2}, \omega\right) .
$$

This equation has the advantage when compared to the previous formulation in space and time domain, that no convolution has to be carried out. Thus, the displacement amplitudes in the frequency-wavenumber domain are related directly to the traction amplitudes for a given set of the circular freqeuncy $\omega$ and the horizontal wavenumbers $k_{1}$ and $k_{2}$ via the Green's function tensor $\bar{G}_{i j}\left(k_{1}, k_{2}, \omega\right)$. When the load in the time domain varies harmonically in the form $p_{i}^{10}\left(x_{1}, x_{2}, t\right)=P_{i}\left(x_{1}, x_{2}\right) \mathrm{e}^{\mathrm{i} \omega t}$, the solution simplifies, since no inverse Fourier transformation over the frequency is necessary. $\bar{G}_{i j}\left(k_{1}, k_{2}, \omega\right)$ must only be evaluated at a single frequency.

The main advantage of the description in the frequency-horizontal wavenumber domain is that a solution for the stratum may be found analytically. In the following subsections, the derivation of $\bar{G}_{i j}\left(k_{1}, k_{2}, \omega\right)$ is described. As mentioned above, the derivation is based on the assumption that the material within each individual layer is linear elastic, homogeneous and isotropic. Further, material dissipation is confined to hysteretic damping, which has been found to be a reasonably accurate model for materials such as soil, even if the model is invalid from a physical point of view. 


\subsection{Flexibility matrix for a single soil layer}

The stratum consists of $J$ horizontally bounded layers, each defined by the Young's modulus $E^{j}$, the Poisson ratio $v^{j}$, the mass density $\rho^{j}$ and the loss factor $\eta^{j}$. Further, the layers have the depths $h^{j}, j=1,2, \ldots, J$. Thus, the equations of motion for each layer may advantageously be established in a coordinate system with the local $x_{3}$-coordinate $x_{3}^{j}$ defined with the positive direction downwards so that $x_{3}^{j} \in\left[0, h^{j}\right]$, see Fig. 3 .

\subsubsection{Boundary conditions for displacements and stresses at an interface}

In the frequency domain, and in terms of the horizontal wavenumbers, the displacements at the top and at the bottom of the $j$ th layer are given, respectively, as

$$
\bar{U}_{i}^{j 0}\left(k_{1}, k_{2}, \omega\right)=\bar{U}_{i}\left(k_{1}, k_{2}, x_{3}^{j}=0, \omega\right), \quad \bar{U}_{i}^{j 1}\left(k_{1}, k_{2}, \omega\right)=\bar{U}_{i}\left(k_{1}, k_{2}, x_{3}^{j}=h^{j}, \omega\right) .
$$

The meaning of the double superscript 10 applied in the definition of the flexibility or Green's function in the previous section now becomes somewhat clearer. Thus $\bar{U}_{i}^{10}$ are the displacement components at the top of the uppermost layer which coincides with the surface of the half-space. The remaining layers are counted downwards with $j=J$ referring to the bottommost layer. If an underlying half-space is present, its material properties are identified by index $j=J+1$.

Similar to Eq. (8) for the displacements, the traction at the top and bottom of layer $j$ are

$$
\bar{P}_{i}^{j 0}\left(k_{1}, k_{2}, \omega\right)=\bar{P}_{i}\left(k_{1}, k_{2}, x_{3}^{j}=0, \omega\right), \quad \bar{P}_{i}^{j 1}\left(k_{1}, k_{2}, \omega\right)=\bar{P}_{i}\left(k_{1}, k_{2}, x_{3}^{j}=h^{j}, \omega\right) .
$$

The quantities defined in Eqs. (8) and (9) may advantageously be stored in vector form as

$$
\overline{\mathbf{S}}^{j 0}=\left[\begin{array}{l}
\overline{\mathbf{U}}^{j 0} \\
\overline{\mathbf{P}}^{j 0}
\end{array}\right], \quad \overline{\mathbf{S}}^{j 1}=\left[\begin{array}{l}
\overline{\mathbf{U}}^{j 1} \\
\overline{\mathbf{P}}^{j 1}
\end{array}\right],
$$

where $\overline{\mathbf{U}}^{j 0}=\overline{\mathbf{U}}^{j 0}\left(k_{1}, k_{2}, \omega\right)$ is the column vector with the components $\bar{U}_{i}^{j 0}, i=1,2,3$, etcetera.

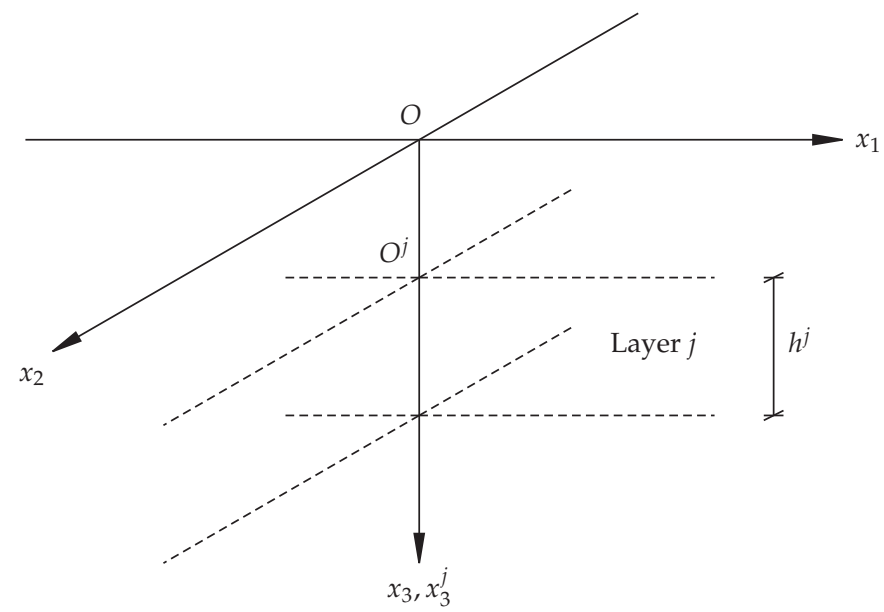

Fig. 3. Global and local coordinates for layer $j$ with the depth $h^{j}$. The $\left(x_{1}, x_{2}, x_{3}\right)$-coordinate system has the origin $O$, whereas the local $\left(x_{1}, x_{2}, x_{3}^{j}\right)$-coordinate system has the origin $O^{j}$. 


\subsubsection{Governing equations for wave propagation in a soil layer}

In the time domain, and in terms of Cartesian coordinates, the equations of motion for the layer are given in terms of the Cauchy equations, which in the absence of body forces read

$$
\frac{\partial}{\partial x_{k}} \sigma_{i k}^{j}\left(x_{1}, x_{2}, x_{3}^{j}, t\right)=\rho^{j} \frac{\partial^{2}}{\partial t^{2}} u_{i}^{j}\left(x_{1}, x_{2}, x_{3}^{j}, t\right),
$$

where $\sigma_{i k}^{j}\left(x_{1}, x_{2}, x_{3}^{j}, t\right)$ is the Cauchy stress tensor. On any part of the boundary, i.e. on the top and bottom of the layer, Dirichlet or Neumann conditions apply as defined by Eqs. (8) and (9), respectively. Initial conditions are of no interest in the present case, since the steady state solution is to be found.

Assuming hysteretic material dissipation defined by the loss factor $\eta^{j}$, the dynamic stiffness of the homogeneous and isotropic material may conveniently be described in terms of complex Lamé constants defined as

$$
\lambda^{j}=\frac{v^{j} E^{j}\left(1+\mathrm{i} \operatorname{sign}(\omega) \eta^{j}\right)}{\left(1+v^{j}\right)\left(1-2 v^{j}\right)}, \quad \mu^{j}=\frac{\left.E^{j}\left(1+\mathrm{i} \operatorname{sign}(\omega) \eta^{j}\right)\right)}{2\left(1+v^{j}\right)} .
$$

The sign function ensures that the material damping is positive in the entire frequency range $\omega \in[-\infty ; \infty]$ involved in the inverse Fourier transformation (3).

Subsequently, the stress amplitudes $\widehat{\sigma}_{i k}^{j}\left(x_{1}, x_{2}, x_{3}^{j}, \omega\right)$ may be expressed in terms of the dilation amplitudes $\widehat{\Delta}^{j}\left(x_{1}, x_{2}, x_{3}^{j}, \omega\right)$, and the infinitesimal strain tensor amplitudes $\widehat{\varepsilon}_{i k}^{j}\left(x_{1}, x_{2}, x_{3}^{j}, \omega\right)$,

$$
\widehat{\sigma}_{i k}^{j}\left(x_{1}, x_{2}, x_{3}^{j}, \omega\right)=\lambda^{j} \widehat{\Delta}^{j}\left(x_{1}, x_{2}, x_{3}^{j}, \omega\right) \delta_{i k}+2 \mu^{j} \widehat{\varepsilon}_{i k}^{j}\left(x_{1}, x_{2}, x_{3}^{j}, \omega\right),
$$

where $\delta_{i j}$ is the Kronecker delta; $\delta_{i j}=1$ for $i=j$ and $\delta_{i j}=0$ for $i \neq j$. Further, the following definitions apply:

$$
\begin{gathered}
\widehat{\Delta}^{j}\left(x_{1}, x_{2}, x_{3}^{j}, \omega\right)=\frac{\partial}{\partial x_{k}} U_{k}^{j}\left(x_{1}, x_{2}, x_{3}^{j}, \omega\right), \\
\widehat{\varepsilon}_{i k}^{j}\left(x_{1}, x_{2}, x_{3}^{j}, \omega\right)=\frac{1}{2}\left(\frac{\partial}{\partial x_{i}} U_{k}^{j}\left(x_{1}, x_{2}, x_{3}^{j}, \omega\right)+\frac{\partial}{\partial x_{k}} U_{i}^{j}\left(x_{1}, x_{2}, x_{3}^{j}, \omega\right)\right) .
\end{gathered}
$$

It is noted that $\partial / \partial x_{3}^{j}=\partial / \partial x_{3}$, since the local $x_{3}^{j}$-axes have the same positive direction as the global $x_{3}$-axis.

Inserting Eqs. (12) to (15) into the Fourier transformation of the Cauchy equation given by Eq. (11), the Navier equations in the frequency domain are achieved:

$$
\left(\lambda^{j}+\mu^{j}\right) \frac{\partial \widehat{\Delta}^{j}}{\partial x_{i}}+\mu^{j} \frac{\partial^{2} U_{i}^{j}}{\partial x_{k} \partial x_{k}}=-\omega^{2} \rho^{j} U_{i}^{j} .
$$

Applying the double Fourier transformation over the horizontal Cartesian coordinates as defined by Eq. (5), the Navier equations in the frequency-wavenumber domain become

$$
\begin{gathered}
\left(\lambda^{j}+\mu^{j}\right) \mathrm{i} k_{i} \bar{\Delta}^{j}+\mu^{j}\left(\frac{\mathrm{d}^{2}}{\mathrm{~d} x_{3}^{2}}-k_{1}^{2}-k_{2}^{2}\right) \bar{U}_{i}^{j}=-\omega^{2} \rho^{j} \bar{U}_{i}^{j}, \quad i=1,2, \\
\left(\lambda^{j}+\mu^{j}\right) \frac{\mathrm{d} \bar{\Delta}^{j}}{\mathrm{~d} x_{3}}+\mu^{j}\left(\frac{\mathrm{d}^{2}}{\mathrm{~d} x_{3}^{2}}-k_{1}^{2}-k_{2}^{2}\right) \bar{U}_{3}^{j}=-\omega^{2} \rho^{j} \bar{U}_{3}^{j},
\end{gathered}
$$


where $\bar{\Delta}^{j}=\bar{\Delta}^{j}\left(k_{1}, k_{2}, x_{3}^{j}, \omega\right)$ is the double Fourier transform of $\widehat{\Delta}^{j}\left(x_{1}, x_{2}, x_{3}^{j}, \omega\right)$ with respect to the horizontal Cartesian coordinates $x_{1}$ and $x_{2}$. Obviously,

$$
\bar{\Delta}^{j}\left(k_{1}, k_{2}, x_{3}^{j}, \omega\right)=\mathrm{i} k_{1} \bar{U}_{1}^{j}\left(k_{1}, k_{2}, x_{3}^{j}, \omega\right)+\mathrm{i} k_{2} \bar{U}_{2}^{j}\left(k_{1}, k_{2}, x_{3}^{j}, \omega\right)+\frac{\mathrm{d} \bar{U}_{3}^{j}\left(k_{1}, k_{2}, x_{3}^{j}, \omega\right)}{\mathrm{d} x_{3}} .
$$

Equations (17a) and (17b) are ordinary differential equations in $x_{3}$. When the boundary values at the top and the bottom of the layer expressed in Eqs. (8) and (9) are known, an analytical solution may be found as will be discussed below.

\subsubsection{The solution for compression waves in a soil layer}

The phase velocities of compression and shear waves, or P- and S-waves, are identified as

$$
c_{P}=\sqrt{\frac{\lambda^{j}+2 \mu^{j}}{\rho^{j}}}, \quad c_{S}=\sqrt{\frac{\mu^{j}}{\rho^{j}}},
$$

respectively. It is noted that the phase velocities are complex when material damping is present. Further, in the frequency domain, the P- and S-waves in layer $j$ are associated with the wavenumbers $k_{P}^{j}$ and $k_{S}^{j}$,

$$
\left\{k_{P}^{j}\right\}^{2}=\frac{\omega^{2}}{\left\{c_{P}^{j}\right\}^{2}}, \quad\left\{k_{S}^{j}\right\}^{2}=\frac{\omega^{2}}{\left\{c_{S}^{j}\right\}^{2}} .
$$

Introducing the parameters $\alpha_{P}^{j}$ and $\alpha_{S}^{j}$ as the larger of the roots to

$$
\left\{\alpha_{P}^{j}\right\}^{2}=k_{1}^{2}+k_{2}^{2}-\left\{k_{P}^{j}\right\}^{2}, \quad\left\{\alpha_{S}^{j}\right\}^{2}=k_{1}^{2}+k_{2}^{2}-\left\{k_{S}^{j}\right\}^{2},
$$

Eqs. (17a) and (17b) may conveniently be recast as

$$
\begin{gathered}
\left(\lambda^{j}+\mu^{j}\right) i k_{i} \bar{\Delta}^{j}+\mu^{j}\left(\frac{\mathrm{d}^{2} U_{i}^{j}}{\mathrm{~d} x_{3}^{2}}-\left\{\alpha_{S}^{j}\right\}^{2} U_{i}^{j}\right)=0, \quad i=1,2, \\
\left(\lambda^{j}+\mu^{j}\right) \frac{\mathrm{d} \bar{\Delta}^{j}}{\mathrm{~d} x_{3}}+\mu^{j}\left(\frac{\mathrm{d}^{2} U_{3}^{j}}{\mathrm{~d} x_{3}^{2}}-\left\{\alpha_{S}^{j}\right\}^{2} U_{3}^{j}\right)=0 .
\end{gathered}
$$

Equation (22a) is now multiplied with $\mathrm{i} k_{i}$ and Eq. (22b) is differentiated with respect to $x_{3}$. Adding the three resulting equations and making use of Eq. (18), an equation for the dilation is obtained in the form

$$
\begin{gathered}
\left(\lambda^{j}+\mu^{j}\right)\left(\frac{\mathrm{d}^{2}}{\mathrm{~d} x_{3}^{2}}-k_{1}^{2}-k_{2}^{2}\right) \bar{\Delta}^{j}+\mu^{j}\left(\frac{\mathrm{d}^{2}}{\mathrm{~d} x_{3}^{2}}-\left\{\alpha_{S}^{j}\right\}^{2}\right) \bar{\Delta}^{j}=0 \quad \Rightarrow \\
\left(\lambda^{j}+2 \mu^{j}\right)\left(\frac{\mathrm{d}^{2}}{\mathrm{~d} x_{3}^{2}}-k_{1}^{2}-k_{2}^{2}\right) \bar{\Delta}^{j}+\mu^{j}\left(k_{1}^{2}+k_{2}^{2}-\left\{\alpha_{S}^{j}\right\}^{2}\right) \bar{\Delta}^{j}=0 \quad \Rightarrow \\
\left(\lambda^{j}+2 \mu^{j}\right)\left(\frac{\mathrm{d}^{2}}{\mathrm{~d} x_{3}^{2}}-k_{1}^{2}-k_{2}^{2}\right) \bar{\Delta}^{j}+\mu^{j}\left\{k_{S}^{j}\right\}^{2} \bar{\Delta}^{j}=0 .
\end{gathered}
$$


The last derivation follows from Eq. (21). Further, Eqs. (19) and (20) involve that

$$
\mu^{j}\left\{k_{S}^{j}\right\}^{2}=\left(\lambda^{j}+2 \mu^{j}\right)\left\{k_{P}^{j}\right\}^{2} .
$$

Inserting this result into Eq. (23), and once again making use of Eq. (21), we finally arrive at the ordinary homogenous differential equation

$$
\frac{\mathrm{d}^{2} \bar{\Delta}^{j}}{\mathrm{~d} x_{3}^{2}}-\left\{\alpha_{P}^{j}\right\}^{2} \bar{\Delta}^{j}=0,
$$

which has the full solution

$$
\bar{\Delta}^{j}=a_{1}^{j} \mathrm{e}^{\alpha_{P}^{j} x_{3}^{j}}+a_{2}^{j} \mathrm{e}^{-\alpha_{P}^{j} x_{3}^{j}} .
$$

Here $a_{1}^{j}$ and $a_{2}^{j}$ are integration constants that follow from the boundary conditions. Physically, the two parts of the solution (26) describe the decay of P-waves travelling in the negative and positive $x_{3}$-direction, respectively, i.e. P-waves moving up and down in the layer.

\subsubsection{The solution for compression and shear waves in a soil layer}

Insertion of the solution (26) into Eqs. (22a) and (22b) leads to three equations for the displacement amplitudes:

$$
\begin{gathered}
\frac{\mathrm{d}^{2} \bar{U}_{i}^{j}}{\mathrm{~d} x_{3}^{2}}-\left\{\alpha_{S}^{j}\right\}^{2} \bar{U}_{i}^{j}=-\left(\frac{\lambda^{j}}{\mu^{j}}+1\right) \mathrm{i} k_{i}\left(a_{1}^{j} \mathrm{e}^{\alpha_{P}^{j} x_{3}^{j}}+a_{2}^{j} \mathrm{e}^{-\alpha_{P}^{j} x_{3}^{j}}\right), \quad i=1,2, \\
\frac{\mathrm{d}^{2} \bar{U}_{3}^{j}}{\mathrm{~d} x_{3}^{2}}-\left\{\alpha_{S}^{j}\right\}^{2} \bar{U}_{3}^{j}=-\left(\frac{\lambda^{j}}{\mu^{j}}+1\right) \alpha_{P}^{j}\left(a_{1}^{j} \mathrm{e}^{\alpha_{P}^{j} x_{3}^{j}}-a_{2}^{j} \mathrm{e}^{-\alpha_{P}^{j} x_{3}^{j}}\right) .
\end{gathered}
$$

Solutions to Eqs. (27a) and (27b) are found in the form

$$
\begin{aligned}
& \bar{U}_{1}^{j}=\bar{U}_{1, c}^{j}+\bar{U}_{1, p}^{j}=b_{1}^{j} \mathrm{e}^{\alpha_{S}^{j} x_{3}^{j}}+b_{2}^{j} \mathrm{e}^{-\alpha_{S}^{j} x_{3}^{j}}+b_{3}^{j} \mathrm{e}^{\alpha_{P}^{j} x_{3}^{j}}+b_{4}^{j} \mathrm{e}^{-\alpha_{P}^{j} x_{3}^{j},} \\
& \bar{U}_{2}^{j}=\bar{U}_{2, c}^{j}+\bar{U}_{2, p}^{j}=c_{1}^{j} \mathrm{e}^{\alpha_{S}^{j} x_{3}^{j}}+c_{2}^{j} \mathrm{e}^{-\alpha_{S}^{j} x_{3}^{j}}+c_{3}^{j} \mathrm{e}^{\alpha_{P}^{j} x_{3}^{j}}+c_{4}^{j} \mathrm{e}^{-\alpha_{P}^{j} x_{3}^{j},} \\
& \bar{U}_{3}^{j}=\bar{U}_{3, c}^{j}+\bar{U}_{3, p}^{j}=d_{1}^{j} \mathrm{e}^{\alpha_{S}^{j} x_{3}^{j}}+d_{2}^{j} \mathrm{e}^{-\alpha_{S}^{j} x_{3}^{j}}+d_{3}^{j} \mathrm{e}^{\alpha_{P}^{j} x_{3}^{j}}+d_{4}^{j} \mathrm{e}^{-\alpha_{P}^{j} x_{3}^{j}},
\end{aligned}
$$

where the subscripts $c$ and $p$ denote the complimentary and the particular solutions, respectively. These include S- and P-wave terms, respectively. Like $a_{1}^{j}$ and $a_{2}^{j}, c_{1}^{j}, c_{2}^{j}$, etc. are integration constants given by the boundary conditions at the top and the bottom of layer $j$. Apparently, the full solution has fourteen integration constants. However, a comparison of Eqs. (18) and (26) reveals that

$$
\bar{\Delta}^{j}\left(k_{1}, k_{2}, x_{3}^{j}, \omega\right)=\mathrm{i} k_{1} \bar{U}_{1}^{j}+\mathrm{i} k_{2} \bar{u}_{2}^{j}+\frac{\mathrm{d} \bar{u}_{3}^{j}}{\mathrm{~d} x_{3}}=a_{1}^{j} \mathrm{e}^{\alpha_{P}^{j} x_{3}^{j}}+a_{2}^{j} \mathrm{e}^{-\alpha_{P}^{j} x_{3}^{j}} .
$$

By insertion of the complementary solutions, i.e. the first two terms in Eqs. (28a) to (28c), into Eq. (29) it immediately follows that

$$
d_{1}^{j}=-\left(\frac{\mathrm{i} k_{1}}{\alpha_{S}^{j}} b_{1}^{j}+\frac{\mathrm{i} k_{2}}{\alpha_{S}^{j}} c_{1}^{j}\right), \quad d_{2}^{j}=\frac{\mathrm{i} k_{1}}{\alpha_{S}^{j}} b_{2}^{j}+\frac{\mathrm{i} k_{2}}{\alpha_{S}^{j}} c_{2}^{j} .
$$


functions of different powers are orthogonal. A further reduction of the number of integration constants is achieved by insertion of the particular solutions into the respective differential equations (27a) and (27b). Thus, after a few manipulations it may be shown that

$$
\begin{aligned}
& b_{3}^{j}=-\frac{\mathrm{i} k_{1}}{\left\{k_{P}^{j}\right\}^{2}} a_{1}^{j}, \quad c_{3}^{j}=-\frac{\mathrm{i} k_{2}}{\left\{k_{P}^{j}\right\}^{2}} a_{1}^{j}, \quad d_{3}^{j}=-\frac{\alpha_{P}^{j}}{\left\{k_{P}^{j}\right\}^{2}} a_{1}^{j}, \\
& b_{4}^{j}=-\frac{\mathrm{i} k_{1}}{\left\{k_{P}^{j}\right\}^{2}} a_{2}^{j}, \quad c_{4}^{j}=-\frac{\mathrm{i} k_{2}}{\left\{k_{P}^{j}\right\}^{2}} a_{2}^{j}, \quad d_{4}^{j}=+\frac{\alpha_{P}^{j}}{\left\{k_{P}^{j}\right\}^{2}} a_{2}^{j},
\end{aligned}
$$

where use has been made of the fact that

$$
\frac{\lambda^{j}+\mu^{j}}{\mu^{j}\left(\left\{\alpha_{S}^{j}\right\}^{2}-\left\{\alpha_{P}^{j}\right\}^{2}\right)}=\frac{\left\{c_{P}^{j}\right\}^{2}-\left\{c_{S}^{j}\right\}^{2}}{\left\{c_{S}^{j}\right\}^{2}\left(\left\{k_{P}^{j}\right\}^{2}-\left\{k_{S}^{j}\right\}^{2}\right)}=\frac{\left\{k_{S}^{j}\right\}^{2}-\left\{k_{P}^{j}\right\}^{2}}{\left\{k_{P}^{j}\right\}^{2}\left(\left\{k_{P}^{j}\right\}^{2}-\left\{k_{S}^{j}\right\}^{2}\right)}=-\frac{1}{\left\{k_{P}^{j}\right\}^{2}},
$$

which follows from the definitions given in Eqs. (19) to (21). Thus, eventually only six of the original fourteen integration constants are independent, namely $a_{1}^{j}, a_{2}^{j}, b_{1}^{j}, b_{2}^{j}, c_{1}^{j}$ and $c_{2}^{j}$. As already mentioned, the terms including $a_{1}^{j}$ and $a_{2}^{j}$ represent P-waves moving up and down in layer $j$. Inspection of Eqs. (28a) to (28c) reveals that the $b_{1}^{j}$ and $b_{2}^{j}$ terms represent $\mathrm{S}$-waves that are polarized in the $x_{1}$-direction and which are moving up and down in the layer, respectively. Similarly, the $c_{1}^{j}$ and $c_{2}^{j}$ terms describe the contributions from S-waves polarized in the $x_{2}$-direction and travelling up and down in the layer, respectively. It becomes evident that the previously defined quantities $\alpha_{P}^{j}$ and $\alpha_{S}^{j}$ may be interpreted as exponential decay coefficients of P- and S-waves, respectively. When $k_{1}$ and $k_{2}$ are both small, $\alpha_{P}^{j}$ and $\alpha_{S}^{j}$ turn into "wavenumbers", as they become imaginary, cf. Eq. (21).

Once the displacement field is known, the stress components on any plane orthogonal to the $x_{3}^{j}$-axis may be found from Eq. (13) by letting index $k=3$. The full solution for displacements, $\overline{\mathbf{U}}^{j}$, and traction, $\overline{\mathbf{P}}^{j}$, may then be written in matrix form as

$$
\overline{\mathbf{S}}^{j}=\left[\begin{array}{c}
\overline{\mathbf{U}}^{j} \\
\overline{\mathbf{P}}^{j}
\end{array}\right]=\mathbf{A}^{j} \mathbf{E}^{j} \mathbf{b}^{j}, \quad \mathbf{b}^{j}=\left[\begin{array}{llllll}
a_{1}^{j} & b_{1}^{j} & c_{1}^{j} & a_{2}^{j} & b_{2}^{j} & c_{2}^{j}
\end{array}\right]^{T},
$$

where $\mathbf{E}^{j}$ is a matrix of dimension $(6 \times 6)$. Only the diagonal terms

$$
E_{11}^{j}=\mathrm{e}^{\alpha_{P}^{j} x_{3}^{j}}, \quad E_{22}^{j}=E_{33}^{j}=\mathrm{e}^{\alpha_{S}^{j} x_{3}^{j}}, \quad E_{44}^{j}=\mathrm{e}^{-\alpha_{P}^{j} x_{3}^{j}}, \quad E_{55}^{j}=E_{66}^{j}=\mathrm{e}^{-\alpha_{S}^{j} x_{3}^{j}},
$$

are nonzero. $\mathbf{A}^{j}$ is a matrix of dimension $(6 \times 6)$, the components of which follow from Eqs. (28) to (31) and (13). The computation of matrix $\mathbf{A}^{j}$ is further discussed below. Finally, the displacements and the traction at the two boundaries of layer $j$ may be expressed as

$$
\begin{array}{ccrl}
\overline{\mathbf{S}}^{j 0} & =\mathbf{A}^{j 0} \mathbf{b}^{j}, & \mathbf{A}^{j 0}=\mathbf{A}^{j}, \\
\overline{\mathbf{S}}^{j 1}=\mathrm{e}^{\alpha_{p}^{j} h^{j}} \mathbf{A}^{j 1} \mathbf{b}^{j}, & \mathbf{A}^{j 1}=\mathbf{A}^{j 0} \mathbf{D}^{j} .
\end{array}
$$

Here $\mathbf{D}^{j}$ a $(6 \times 6)$ matrix with the nonzero components

$$
D_{11}^{j}=1, D_{22}^{j}=D_{33}^{j}=\mathrm{e}^{\left(\alpha_{S}^{j}-\alpha_{P}^{j}\right) h^{j}}, D_{44}^{j}=\mathrm{e}^{-2 \alpha_{P}^{j} h^{j}}, D_{55}^{j}=D_{66}^{j}=\mathrm{e}^{-\left(\alpha_{P}^{j}+\alpha_{S}^{j}\right) h^{j}},
$$


found by evaluation of the matrix $\mathrm{e}^{-\alpha_{P}^{j} x_{3}^{j}} \mathbf{E}^{j}$ at $x_{3}^{j}=h^{j}$. Equations (34a) and (34b) may be combined in order to eliminate vector $\mathbf{b}^{j}$ which contains unknown integration constants. This provides a transfer matrix for the layer as proposed by Thomson (1950) and Haskell (1953),

$$
\overline{\mathbf{S}}^{j 1}=\mathrm{e}^{\alpha_{P}^{j} h^{j}} \mathbf{A}^{j 1}\left[\mathbf{A}^{j 0}\right]^{-1} \overline{\mathbf{S}}^{j 0},
$$

forming a relationship between the displacements and the traction at the top and the bottom of a single layer.

The derivation of Eq. (36) has been based on the assumption that $\omega>0$. When a static load is applied, the circular frequency is $\omega=0$, whereby the wavenumbers of the P-and S-waves, i.e. $k_{P}^{j}$ and $k_{S}^{j}$ defined by Eq. (20), become zero and the integration constants $b_{3}^{j}$ etc. given in Eq. (31) are undefined. Hence, the solution given in the previous section does not apply in the static case. However, for any practical purposes a useful approximation can be established for the static case by employing a low value of $\omega$ in the evaluation of $\overline{\mathbf{S}}^{j 1}$.

\subsection{Assembly of multiple layers}

At an interface between two layers, the displacements should be continuous and there should be equilibrium of the traction. This may be expressed as $\overline{\mathbf{S}}^{j 0}=\overline{\mathbf{S}}^{j-1,1}, j=2,3, \ldots, J$, i.e. the quantities at the top of layer $j$ are equal to those at the bottom of layer $j-1$. Proceeding in this manner, Eq. (36) for the single layer may be rewritten for a system of $J$ layers,

$$
\overline{\mathbf{S}}^{J 1}=\mathrm{e}^{\Sigma \alpha} \mathbf{A}^{J 1}\left[\mathbf{A}^{10}\right]^{-1} \mathbf{A}^{J-1,1}\left[\mathbf{A}^{J-1,0}\right]^{-1} \cdots \mathbf{A}^{11}\left[\mathbf{A}^{10}\right]^{-1} \overline{\mathbf{S}}^{10}, \quad \Sigma \alpha=\sum_{j=1}^{J} \alpha_{P}^{j} h^{j} .
$$

Introducing the transfer matrix $\mathbf{T}$ defined as

$$
\mathbf{T}=\left[\begin{array}{ll}
\mathbf{T}_{11} & \mathbf{T}_{12} \\
\mathbf{T}_{21} & \mathbf{T}_{22}
\end{array}\right]=\mathbf{A}^{J 1}\left[\mathbf{A}^{J 0}\right]^{-1} \mathbf{A}^{J-1,1}\left[\mathbf{A}^{J-1,0}\right]^{-1} \cdots \mathbf{A}^{11}\left[\mathbf{A}^{10}\right]^{-1},
$$

Equation (37) may in turn be written as $\overline{\mathbf{S}}^{J 1}=\mathrm{e}^{\Sigma \alpha} \mathbf{T} \overline{\mathbf{S}}^{10}$, or

$$
\left[\begin{array}{l}
\overline{\mathbf{U}}^{J 1} \\
\overline{\mathbf{P}}^{J 1}
\end{array}\right]=\mathrm{e}^{\Sigma \alpha}\left[\begin{array}{ll}
\mathbf{T}_{11} & \mathbf{T}_{12} \\
\mathbf{T}_{21} & \mathbf{T}_{22}
\end{array}\right]\left[\begin{array}{l}
\overline{\mathbf{U}}^{10} \\
\overline{\mathbf{P}}^{10}
\end{array}\right], \quad \sum \alpha=\sum_{j=1}^{J} \alpha_{P}^{j} h^{j} .
$$

This establishes a relationship between the traction and the displacements at the free surface of the half-space and the equivalent quantities at the bottom of the stratum as originally proposed by Thomson (1950) and Haskell (1953).

\subsection{Flexibility of a homogeneous or stratified ground}

A stratified ground consisting of multiple soil layers may overlay bedrock. On the surface of the bedrock, the displacements are identically equal to zero and thus, by insertion into Eq. (39),

$$
\left[\begin{array}{c}
\overline{\mathbf{U}}^{J 1} \\
\overline{\mathbf{P}}^{J 1}
\end{array}\right]=\left[\begin{array}{c}
\mathbf{0} \\
\overline{\mathbf{P}}^{I 1}
\end{array}\right]=\mathrm{e}^{\Sigma \alpha}\left[\begin{array}{ll}
\mathbf{T}_{11} & \mathbf{T}_{12} \\
\mathbf{T}_{21} & \mathbf{T}_{22}
\end{array}\right]\left[\begin{array}{l}
\overline{\mathbf{U}}^{10} \\
\overline{\mathbf{P}}^{10}
\end{array}\right] \text {. }
$$

The first three rows of this matrix equation provide the identity

$$
\overline{\mathbf{U}}^{10}=\overline{\mathrm{G}}_{\mathrm{rf}} \overline{\mathbf{P}}^{10}, \quad \overline{\mathrm{G}}_{\mathrm{rf}}=-\mathbf{T}_{11}^{-1} \mathbf{T}_{12} .
$$


$\overline{\mathrm{G}}_{\mathrm{rf}}=\overline{\mathrm{G}}_{\mathrm{rf}}\left(k_{1}, k_{2}, \omega\right)$ is the flexibility matrix for a stratum over a rigid bedrock. It is observed that the exponential function of the power $\Sigma \alpha$, defined in Eq. (39), vanishes in the formulation provided by Eq. (41). This is a great advantage from a computational point of view, since $\mathrm{e}^{\Sigma \alpha}$ becomes very large for strata of great depths, which may lead to problems on a computer-even when double precision complex variables are employed.

Alternatively to a rigid bedrock, a half-space may be present underneath the stratum consisting of $J$ layers. In this context, the material properties etc. of the half-space will be assigned the superscript $J+1$. The main difference between a semi-infinite half-space and a layer of finite depth is that only an upper boundary is present, i.e. the boundary situated at $x_{3}^{J+1}=0$. Since the material is assumed to be homogeneous, no reflection of waves will take place inside the half-space. Further assuming that no sources are present in the interior of the half-space, only outgoing, i.e. downwards propagating, waves can be present. Dividing the matrices $\mathbf{A}^{j}$ and $\mathbf{E}^{j}$ for a layer of finite depth, cf. Eq. (32), into four quadrants, and the column vector $\mathbf{b}^{j}$ into two sub-vectors,

$$
\mathbf{A}^{j}=\left[\begin{array}{ll}
\mathbf{A}_{11}^{j} & \mathbf{A}_{12}^{j} \\
\mathbf{A}_{21}^{j} & \mathbf{A}_{22}^{j}
\end{array}\right], \quad \mathbf{E}^{j}=\left[\begin{array}{ll}
\mathbf{E}_{11}^{j} & \mathbf{E}_{12}^{j} \\
\mathbf{E}_{21}^{j} & \mathbf{E}_{22}^{j}
\end{array}\right], \quad \mathbf{b}^{j}=\left[\begin{array}{c}
\mathbf{b}_{1}^{j} \\
\mathbf{b}_{2}^{j}
\end{array}\right],
$$

it is evident that only half of the solution applies to the half-space, i.e.

$$
\overline{\mathbf{S}}^{J+1}=\left[\begin{array}{l}
\overline{\mathbf{U}}^{J+1} \\
\overline{\mathbf{P}}^{J+1}
\end{array}\right]=\left[\begin{array}{l}
\mathbf{A}_{22}^{J+1} \\
\mathbf{A}_{22}^{++1}
\end{array}\right] \mathbf{E}_{22}^{J+1} \mathbf{b}_{2}^{J+1}, \quad \mathbf{b}_{2}^{J+1}=\left[\begin{array}{lll}
a_{2}^{J+1} & b_{2}^{J+1} & c_{2}^{J+1}
\end{array}\right]^{T} .
$$

The terms including the integration constants $a_{1}^{J+1}, b_{1}^{J+1}$ and $c_{1}^{J+1}$ are physically invalid as they correspond to waves incoming from $x_{3}^{J+1}=\infty$, i.e. from infinite depth.

From Eq. (43), the traction on the interface between the bottommost layer and the half-space may be expressed in terms of the corresponding displacements by solution of

$$
\overline{\mathbf{U}}^{J+1}=\mathbf{A}_{12}^{J+1}\left[\mathbf{A}_{22}^{J+1}\right]^{-1} \overline{\mathbf{P}}^{J+1} .
$$

The matrix $\mathbf{E}_{22}^{J+1}$ reduces to the identity matrix of order 3 , since all the exponential terms are equal to 1 for $x_{3}^{J+1}=0$.

Firstly, if no layers are present in the model of the stratum, $J=0$ and it immediately follows from Eq. (44) that Eq. (7), written in matrix form, becomes

$$
\overline{\mathbf{U}}^{10}=\overline{\mathbf{G}}_{\mathbf{h h}} \overline{\mathbf{P}}^{10}, \quad \overline{\mathbf{G}}_{\mathbf{h h}}=\mathbf{A}_{12}^{10}\left[\mathbf{A}_{22}^{10}\right]^{-1},
$$

where it is noted that the flexibility matrix for the homogeneous half-space $\overline{\mathrm{G}}_{\mathrm{hh}}=$ $\overline{\mathbf{G}}_{\mathbf{h h}}\left(k_{1}, k_{2}, \omega\right)$ is given in the horizontal wavenumber-frequency domain.

Secondly, when $J$ layers overlay a homogeneous half-space, continuity of the displacements, equilibrium of the traction and application of Eq. (44) provide

$$
\overline{\mathbf{U}}^{J 1}=\overline{\mathbf{U}}^{J+1,0}=\mathbf{A}_{12}^{J+1}\left[\mathbf{A}_{22}^{J+1}\right]^{-1} \overline{\mathbf{P}}^{J+1,0}=\mathbf{A}_{12}^{J+1}\left[\mathbf{A}_{22}^{J+1}\right]^{-1} \overline{\mathbf{P}}^{J 1} .
$$

Insertion of this result into Eq. (39) leads to the following system of equations:

$$
\left[\begin{array}{l}
\overline{\mathbf{U}}^{J 1} \\
\overline{\mathbf{P}}^{J 1}
\end{array}\right]=\left[\begin{array}{rr}
\mathbf{A}_{12}^{J+1}\left[\mathbf{A}_{22}^{J+1}\right]^{-1} \overline{\mathbf{P}}^{J 1} \\
\overline{\mathbf{P}}^{J 1}
\end{array}\right]=\mathrm{e}^{\Sigma \alpha}\left[\begin{array}{ll}
\mathbf{T}_{11} & \mathbf{T}_{12} \\
\mathbf{T}_{21} & \mathbf{T}_{22}
\end{array}\right]\left[\begin{array}{l}
\overline{\mathbf{U}}^{10} \\
\overline{\mathbf{P}}^{10}
\end{array}\right] .
$$


From the bottommost three rows of the matrix equation, an expression of $\overline{\mathbf{P}}^{J 1}$ is obtained which may be inserted into the first three equations. This leads to the solution

$$
\overline{\mathbf{U}}^{10}=\overline{\mathbf{G}}_{\mathbf{l h}} \overline{\mathbf{P}}^{10}
$$

where the flexibility matrix for the layered half-space $\overline{\mathbf{G}}_{\mathbf{l h}}=\overline{\mathbf{G}}_{\mathbf{l h}}\left(k_{1}, k_{2}, \omega\right)$ is given by

$$
\overline{\mathbf{G}}_{\mathbf{l h}}=\left(\mathbf{A}_{12}^{J+1}\left[\mathbf{A}_{22}^{J+1}\right]^{-1} \mathbf{T}_{21}-\mathbf{T}_{11}\right)^{-1}\left(\mathbf{T}_{12}-\mathbf{A}_{12}^{J+1}\left[\mathbf{A}_{22}^{J+1}\right]^{-1} \mathbf{T}_{22}\right) .
$$

Again the exponential function disappears. In the following, no distinction is made between $\overline{\mathrm{G}}_{\mathrm{rf}}, \overline{\mathrm{G}}_{\mathrm{hh}}$ and $\overline{\mathrm{G}}_{\mathrm{lh}}$. The common notation $\overline{\mathrm{G}}$ will be employed, independent of the type of subsoil model.

\subsection{Optimising the numerical evaluation of the Green's function}

In order to obtain a solution in Cartesian space, a double inverse Fourier transformation over the horizontal wavenumbers is necessary as outlined by Eq. (6). A direct approach involves the evaluation of $\overline{\mathbf{G}}$ for numerous combinations of $k_{1}$ and $k_{2}$, leading to long computation times. However, as described in this subsection, a considerable reduction of the computation time can be achieved.

\subsubsection{Computation of the matrices $\mathrm{A}^{j 0}$ and $\mathrm{A}^{j 1}$}

The computation of the transfer matrix $\mathbf{T}$ involves inversion of the matrices $\mathbf{A}^{j 0}, j=1,2, \ldots, J$. Further, the flexibility matrix $\overline{\mathbf{G}}\left(k_{1}, k_{2}, \omega\right)$ has to be evaluated for all combinations $\left(k_{1}, k_{2}\right)$ before the transformation given by Eq. (6) may be applied. However, as pointed out by Sheng et al. (1999), the evaluation of $\mathbf{A}^{j}$, and therefore also the Green's function matrix $\overline{\mathbf{G}}$, is particularly simple along the line defined by $k_{1}=0$. To take advantage of this, a coordinate transformation is introduced in the form

$$
\left[\begin{array}{l}
k_{1} \\
k_{2} \\
x_{3}
\end{array}\right]=\mathbf{R}(\varphi)\left[\begin{array}{l}
\gamma \\
\alpha \\
x_{3}
\end{array}\right], \quad \mathbf{R}(\varphi)=\left[\begin{array}{ccc}
\sin \varphi & \cos \varphi & 0 \\
-\cos \varphi & \sin \varphi & 0 \\
0 & 0 & 1
\end{array}\right] .
$$

This corresponds to a rotation of $\left(k_{1}, k_{2}, x_{3}\right)$-basis by the angle $\varphi-\pi / 2$ around the $x_{3}$-axis as illustrated in Fig. 4. It follows from Eq. (50) that $R_{i j}(\varphi)=R_{j i}(\pi-\varphi)$, which in matrix-vector notation corresponds to $\{\mathbf{R}(\varphi)\}^{T}=\mathbf{R}(\pi-\varphi)$.

For any combination of $k_{1}$ and $k_{2}$, the angle $\varphi$ is now defined so that $\gamma=0$. The relationship between the coordinates in the two systems of reference is then given by

$$
k_{1}=\alpha \cos \varphi, \quad k_{2}=\alpha \sin \varphi, \quad \alpha=\sqrt{k_{1}^{2}+k_{2}^{2}}, \quad \tan \varphi=\frac{k_{2}}{k_{1}}, \quad \gamma=0 .
$$

The computational advantage of this particular orientation of the $\left(\gamma, \alpha, x_{3}\right)$-coordinate system is twofold. Firstly, the flexibility matrix may be evaluated along a line rather than over an area, and for any other combination of the wavenumbers, the Green's function matrix can be computed as

$$
\overline{\mathbf{G}}\left(k_{1}, k_{2}, \omega\right)=\mathbf{R}(\varphi) \widehat{\overline{\mathbf{G}}}\{\mathbf{R}(\varphi)\}^{T} \quad \text { or } \quad \bar{G}_{i k}\left(k_{1}, k_{2}, \omega\right)=R_{i l}(\varphi) \widehat{\bar{G}}_{l m} R_{k m}(\varphi) .
$$




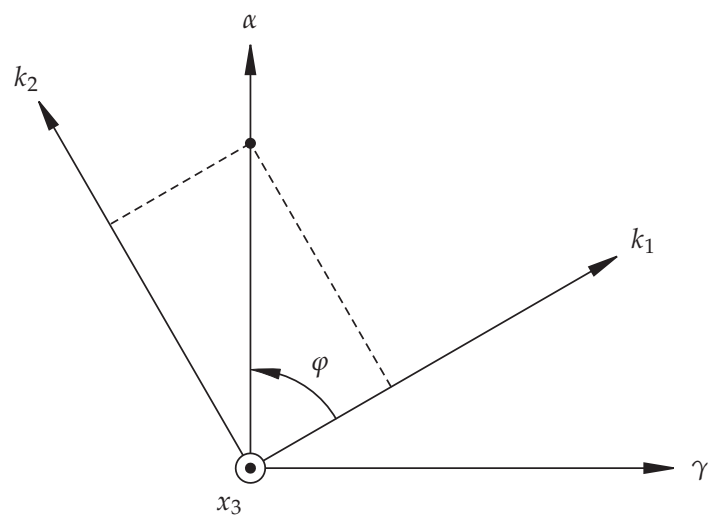

Fig. 4. Definition of the $\left(k_{1}, k_{2}, x_{3}\right)$ - and $\left(\gamma, \alpha, x_{3}\right)$-coordinate systems.

Here $\widehat{\overline{\mathbf{G}}}=\widehat{\overline{\mathbf{G}}}(\alpha, \omega)=\overline{\mathbf{G}}(0, \alpha, \omega)$. Secondly, the matrices $\mathbf{A}^{j 0}$ and $\mathbf{A}^{j 1}$-and therefore also $\mathbf{A}_{12}^{J+1}$ and $\mathbf{A}_{22}^{J+1}$ - simplify significantly when one of the wavenumbers is equal to zero. Thus, when $k_{1}=\gamma=0, k_{2}=\alpha$ and $\omega \neq 0$,

$$
\widehat{\mathbf{A}}^{j 0}=\mathbf{A}^{j 0}(0, \alpha, \omega)=\left[\begin{array}{cccccc}
0 & 1 & 0 & 0 & 1 & 0 \\
\widehat{A}_{21}^{j 0} & 0 & 1 & \widehat{A}_{21}^{j 0} & 0 & 1 \\
\widehat{A}_{31}^{j 0} & 0 & \widehat{A}_{33}^{j 0} & -\widehat{A}_{31}^{j 0} & 0 & -\widehat{A}_{33}^{j 0} \\
0 & \widehat{A}_{42}^{j 0} & 0 & 0 & -\widehat{A}_{42}^{j 0} & 0 \\
\widehat{A}_{51}^{j 0} & 0 & \widehat{A}_{53}^{j 0} & -\widehat{A}_{51}^{j 0} & 0 & -\widehat{A}_{53}^{j 0} \\
\widehat{A}_{61}^{j 0} & 0 & \widehat{A}_{63}^{j 0} & \widehat{A}_{61}^{j 0} & 0 & \widehat{A}_{63}^{j 0}
\end{array}\right],
$$

where

$$
\begin{gathered}
\widehat{A}_{21}^{j 0}=-\mathrm{i} \alpha /\left\{k_{P}^{j}\right\}^{2}, \quad \widehat{A}_{31}^{j 0}=-\alpha_{P}^{j} /\left\{k_{P}^{j}\right\}^{2}, \quad \widehat{A}_{33}^{j 0}=-\mathrm{i} \alpha / \alpha_{S}^{j}, \\
\widehat{A}_{42}^{j 0}=\alpha_{S}^{j} \mu^{j}, \quad \widehat{A}_{51}^{j 0}=-2 \mathrm{i} \mu^{j} \alpha_{P}^{j} \alpha /\left\{k_{P}^{j}\right\}^{2}, \quad \widehat{A}_{53}^{j 0}=\mu^{j}\left(\alpha^{2} / \alpha_{S}^{j}+\alpha_{S}^{j}\right), \\
\widehat{A}_{61}^{j 0}=-\mu^{j}\left(\left\{k_{S}^{j}\right\}^{2}+2\left\{\alpha_{S}^{j}\right\}^{2}\right) /\left\{k_{P}^{j}\right\}^{2}, \quad \widehat{A}_{63}^{j 0}=-2 \mathrm{i} \mu^{j} \alpha .
\end{gathered}
$$

At the bottom of the layer, the corresponding matrix is evaluated as $\widehat{\mathbf{A}}^{j 1}=\widehat{\mathbf{A}}^{j 0} \mathbf{D}^{j}$, where the components of the matrix $\mathbf{D}^{j}$ are given by Eq. (35). A result of the many zeros in $\widehat{\mathbf{A}}^{j 0}$ and $\widehat{\mathbf{A}}_{0}^{j 0}$ is that the matrices can be inverted analytically. This may reduce computation time significantly. The inversion of $\widehat{\mathbf{A}}^{j 0}$ and $\widehat{\mathbf{A}}_{0}^{j 0}$ is straightforward and will not be treated further.

Especially, for a homogeneous half-space, possibly underlying a stratum, the matrices $\widehat{\mathbf{A}}_{12}^{J+1,0}$ and $\widehat{\mathbf{A}}_{22}^{J+1,0}$ are readily obtained from the leftmost three columns of $\widehat{\mathbf{A}}^{j 0}$, whereas $\widehat{\mathbf{A}}^{j 1}$ is obtained as

$$
\widehat{\mathbf{A}}^{j 1}=\widehat{\mathbf{A}}^{j 0} \mathbf{D}^{j}
$$

in accordance with Eq. (34b). Note that $\mathbf{D}^{j}$ is symmetric in the $\left(k_{1}, k_{2}\right)$-plane and that therefore $\widehat{\mathbf{D}}^{j}=\mathbf{D}^{j}$. This property follows from the definition of the exponential decay coefficients $\alpha_{P}^{j}$ and $\alpha_{S}^{j}$ given in Eq. (21), or the definition of $\alpha$ given by Eq. (51), along with the definition of $\mathbf{D}^{j}$, cf. Eq. (35). In other words it may be stated that $\alpha_{P}^{j}$ and $\alpha_{S}^{j}$ are invariant to rotation around 
the $x_{3}$-axis. As was the case with the matrices for a stratum, the inversion of the matrix $\widehat{\mathbf{A}}_{22}^{J+1,0}$ can be expressed analytically. This mathematical exercise is left to the reader.

\subsubsection{Interpolation of the one-dimensional wavenumber spectrum}

As mentioned above, a direct evaluation of $\bar{G}$ involves a computation over the entire $\left(k_{1}, k_{2}\right)$-space. Making use of the coordinate transformation, the problem is reduced by one dimension, since $\widehat{\bar{G}}$ needs only be evaluated along the $\alpha$-axis. The following procedure is suggested:

1. $\widehat{\overline{\mathrm{G}}}$ is computed for $\alpha=0, \Delta \alpha, 2 \Delta \alpha, \ldots, N \Delta \alpha$. Here $\Delta \alpha$ must be sufficiently small to ensure that local peaks in the Green's function are described. $N$ must be sufficiently large so that $\widehat{\overline{\mathrm{G}}}(\alpha, \omega) \approx 0$ for $\alpha>N \Delta \alpha$.

2. The values of $\widehat{\overline{\mathbf{G}}}(\alpha, \omega)$ for $\alpha=\sqrt{k_{1}^{2}+k_{2}^{2}}$ are computed by linear interpolation between the values obtained at the $N+1$ discrete points.

3. Before the double Fourier transformation given by Eq. (6) is carried out, the coordinate transformation is applied.

In order to provide a fast computation of the inverse Fourier transformation it may be advantageous to use $N=2^{n}$ wavenumbers in either direction so that that an inverse fast Fourier transformation (iFFT) procedure may be applied. The iFFT provides an efficient transformation of the entire discrete field $\bar{U}_{i}^{10}\left(k_{1}, k_{2}, \omega\right)$ into the entire discrete field $U_{i}^{10}\left(x_{1}, x_{2}, \omega\right)$. Given that the wavenumber step is $\Delta \alpha$, the area covered in Cartesian space becomes $2 \pi / \Delta \alpha \times 2 \pi / \Delta \alpha$. Since the number of points on the surface in either coordinate direction in the Cartesian space is identical to the number of points $N$ in the wavenumber domain, the spatial increment $\Delta x=2 \pi /(N \Delta \alpha)$.

In numerical methods based on a spatial discretization, e.g. the FEM, the BEM or finite differences, at least 5-10 points should be present per wavelength in order to provide an accurate solution. However, in the domain transformation method, the requirement is that the Fourier transformed field is described with satisfactory accuracy in the wavenumber domain. If the results in Cartesian coordinates are subsequently only evaluated at a few points per wavelength, this will only mean that the wave field does not become visible- the few responses that are computed will still be accurate. This is a great advantage when dealing with high frequencies. It has been found that $2048 \times 2048$ wavenumbers are required in order to give a sufficiently accurate description of the response Sheng et al. (1999). On the other hand, if the displacements are only to be computed over an area which is much smaller than the area spanned by the wavenumbers, say at a few points, it may be more efficient to use the discretized version of Eq. (6) directly.

\subsubsection{Evaluation of the response in cylindrical coordinates}

As discussed on p. 10, the matrices $\mathbf{A}^{j 0}$ and $\mathbf{A}^{j 1}$ define a relationship between the tractions and displacements at the top and bottom of a viscoelastic layer. The six columns/rows of these matrices correspond to a decomposition of the displacement field into P-waves and S-waves polarized in the $x_{1}$ - and $x_{2}$-directions, respectively, and moving up or down through the layer. Firstly, consider a vertical source or a horizontal source acting in the $\alpha$-direction, i.e. along the axis forming the angle $\varphi-\pi / 2$ with the $k_{1}$-axis around the $x_{3}$-axis, see Fig. 4 . This source produces P- and SV-waves, i.e. S-waves polarised in the vertical direction. Secondly, if a 
source is applied in the transverse direction (the $\gamma$-direction) only $\mathrm{SH}$-waves are generated, i.e. S-waves polarised in the horizontal direction. These propagate in a stratum independently of the two other wave types. Therefore, the Green's function $\widehat{\bar{G}}(\alpha, \omega)$ simplifies to the form

$$
\widehat{\overline{\mathbf{G}}}(\alpha, \omega)=\left[\begin{array}{ccc}
\hat{\bar{G}}_{11} & 0 & 0 \\
0 & \widehat{\bar{G}}_{22} & \widehat{\bar{G}}_{23} \\
0 & \widehat{\bar{G}}_{32} & \widehat{\bar{G}}_{33}
\end{array}\right]
$$

with the zeros indicating the missing interaction between SH-waves and P- and SV-waves. This is exactly the result provided by Eqs. (45) and (49) for a homogeneous and stratified half-space, respectively, after insertion of the matrices $\widehat{\mathbf{A}}^{j 0}, \widehat{\mathbf{A}}_{0}^{j 1}$, etc.. Further, due to reciprocity the matrix $\widehat{\bar{G}}(\alpha, \omega)$ is generally antisymmetric, i.e. $\widehat{\bar{G}}_{32}=-\widehat{\bar{G}}_{23}$, cf. Auersch (1988). As discussed above, $\overline{\mathbf{G}}=\mathbf{R} \widehat{\overline{\mathbf{G}}} \mathbf{R}^{T}$, where $\mathbf{R}=\mathbf{R}(\varphi)$ is the transformation matrix defined in Eq. (50). Hence, the displacement response in the horizontal wavenumber domain may be found as

$$
\bar{U}_{i}^{10}=R_{i j}(\varphi) \widehat{\bar{G}}_{j k}(\alpha, \omega) R_{l k}(\varphi) \bar{P}_{l}^{10},
$$

where $\bar{U}_{i}^{10}=\bar{U}_{i}^{10}\left(k_{1}, k_{2}, \omega\right)=\bar{U}_{i}^{10}(\alpha \cos \varphi, \alpha \sin \varphi, \omega)$ and a similar definition applies to $\bar{P}_{l}^{10}$. Similarly to the transformation of the horizontal wavenumbers from $\left(k_{1}, k_{2}\right)$ into $(\gamma, \alpha)$, the Cartesian coordinate system is rotated around the $x_{3}$-axis according to transformation

$$
\left[\begin{array}{l}
x_{1} \\
x_{2} \\
x_{3}
\end{array}\right]=\mathbf{R}\left[\begin{array}{c}
q \\
r \\
x_{3}
\end{array}\right], \quad \mathbf{R}=\mathbf{R}(\theta)=\left[\begin{array}{rrr}
\sin \theta & \cos \theta & 0 \\
-\cos \theta & \sin \theta & 0 \\
0 & 0 & 1
\end{array}\right] .
$$

The displacement amplitude vector in $\left(q, r, x_{3}\right)$-coordinates is denoted $\widehat{\mathbf{U}}\left(q, r, x_{3}\right)$ and has the components $\left(\widehat{U}_{q}, \widehat{U}_{r}, \widehat{U}_{3}\right)$. Likewise, the load amplitudes are represented by the vector $\widehat{\mathbf{P}}\left(q, r, x_{3}\right)$ with components $\left(\widehat{P}_{q}, \widehat{P}_{r}, \widehat{P}_{3}\right)$. According to Eq. (57) the corresponding amplitudes in the Cartesian $\left(x_{1}, x_{2}, x_{3}\right)$-coordinates are given as

$$
\mathbf{U}\left(x_{1}, x_{2}, x_{3}\right)=\mathbf{R}(\theta) \widehat{\mathbf{U}}\left(q, r, x_{3}\right), \quad \mathbf{P}\left(x_{1}, x_{2}, x_{3}\right)=\mathbf{R}(\theta) \widehat{\mathbf{P}}\left(q, r, x_{3}\right) .
$$

For a given observation point $\left(x_{1}, x_{2}, 0\right)$ on the surface of the half-space, the angle $\theta$ is now selected so that $q=0$, i.e. the point lies on the $r$-axis. Hence, the response to a load applied over an area of rotational symmetry around the $x_{3}$-axis may be evaluated in cylindrical coordinates,

$$
x_{1}=r \cos \theta, \quad x_{2}=r \sin \theta, \quad r=\sqrt{x_{1}^{2}+x_{2}^{2}}, \quad \tan \theta=\frac{x_{2}}{x_{1}} .
$$

Thus, at any given point $\widehat{U}_{r}\left(0, r, x_{3}\right)$ is the radial displacement amplitude whereas $\widehat{U}_{q}\left(0, r, x_{3}\right)$ is the amplitude of the displacement in the tangential direction.

The coordinate transformations (50) and (57) are defined by two angles. Thus, $\varphi$ defines the rotation of the wavenumber $\left(k_{1}, k_{2}\right)$ aligned with the Cartesian $\left(x_{1}, x_{2}\right)$-coordinates into the rotated wavenumbers $(\gamma, \alpha)$. Likewise, a transformation of the Cartesian coordinates $\left(x_{1}, x_{2}\right)$ into the rotated $(q, r)$-coordinate frame is provided by the angle $\theta$. However, in order to simplify the analysis in cylindrical coordinates, it is convenient to introduce the angle

$$
\vartheta=\pi / 2+\varphi-\theta
$$


defining the rotation of the wavenumbers $(\gamma, \alpha)$ relative to the spatial coordinates $(q, r)$. The transformation is illustrated in Fig. 5. Evidently $\mathbf{R}(\varphi)=\mathbf{R}(\theta) \mathbf{R}(\vartheta)$, and the wavenumbers $\left(k_{1}, k_{2}\right)$ in the original Cartesian frame of reference may be obtained from the rotated wavenumbers $(\gamma, \alpha)$ by either of the transformations

$$
\left[\begin{array}{l}
k_{1} \\
k_{2} \\
x_{3}
\end{array}\right]=\mathbf{R}(\varphi)\left[\begin{array}{c}
\gamma \\
\alpha \\
x_{3}
\end{array}\right]=\mathbf{R}(\theta) \mathbf{R}(\vartheta)\left[\begin{array}{l}
\gamma \\
\alpha \\
x_{3}
\end{array}\right], \quad \mathbf{R}(\vartheta)=\left[\begin{array}{ccc}
\sin \vartheta & \cos \vartheta & 0 \\
-\cos \vartheta & \sin \vartheta & 0 \\
0 & 0 & 1
\end{array}\right] .
$$

This identity is easily proved by combination of Eqs. (50), (57), (60) and (61).

Firstly, by application of the coordinate transformation (57) in Eq. (6), the response at the surface of the stratum may be evaluated by a double inverse Fourier transform in polar coordinates, here given in matrix form

$$
\widehat{\mathbf{U}}^{10}=\frac{1}{4 \pi^{2}} \int_{0}^{\infty} \int_{0}^{2 \pi} \mathbf{R}(\vartheta) \widehat{\overline{\mathbf{G}}}\{\mathbf{R}(\vartheta)\}^{T} \widehat{\overline{\mathbf{P}}}^{10} \mathrm{e}^{\mathrm{i} \alpha r \sin \vartheta} \mathrm{d} \vartheta \alpha \mathrm{d} \alpha,
$$

where $\alpha r \sin \vartheta=k_{1} x_{1}+k_{2} x_{2}$ is identified as the dot product of the two-dimensional vectors with lengths $\alpha$ and $r$, respectively, and $\pi / 2-\vartheta$ is the plane angle between these vectors as given by Eq. (60). In accordance with Eq. (58), the load amplitudes given in terms of $x_{3}$ and the horizontal wavenumbers $\left(k_{q}, k_{r}\right)$ are found from the corresponding load amplitudes in $\left(k_{1}, k_{2}, x_{3}\right)$-space by means of the transformation $\widehat{\mathbf{P}}\left(k_{q}, k_{r}, x_{3}\right)=\{\mathbf{R}(\theta)\}^{T} \mathbf{P}\left(k_{1}, k_{2}, x_{3}\right)$. Furthermore, transformation of the displacement amplitudes from $\left(q, r, x_{3}\right)$-coordinates into $\left(x_{1}, x_{2}, x_{3}\right)$-coordinates provides the double inverse Fourier transformation

$$
\mathbf{U}^{10}=\frac{1}{4 \pi^{2}} \mathbf{R}(\theta) \int_{0}^{\infty} \int_{0}^{2 \pi} \mathbf{R}(\vartheta) \widehat{\overline{\mathbf{G}}}\{\mathbf{R}(\vartheta)\}^{T}\{\mathbf{R}(\theta)\}^{T} \overline{\mathbf{P}}^{10} \mathrm{e}^{\mathrm{i} \alpha r \sin \vartheta} \mathrm{d} \vartheta \alpha \mathrm{d} \alpha .
$$

The component form of Eq. (63) reads

$$
U_{i}^{10}=\frac{R_{i k}(\theta)}{4 \pi^{2}} \int_{0}^{\infty} \int_{0}^{2 \pi} R_{k l}(\vartheta) \widehat{\bar{G}}_{l m}(0, \alpha, \omega) R_{n m}(\vartheta) R_{j n}(\theta) \bar{P}_{j}^{10} \mathrm{e}^{\mathrm{i} \alpha r \sin \vartheta} \mathrm{d} \vartheta \alpha \mathrm{d} \alpha .
$$

If summation is skipped over index $j$, this defines the displacement in direction $i$ at a point $\left(x_{1}, x_{2}, 0\right)$ on the surface of the stratified or homogeneous ground due to a load applied in

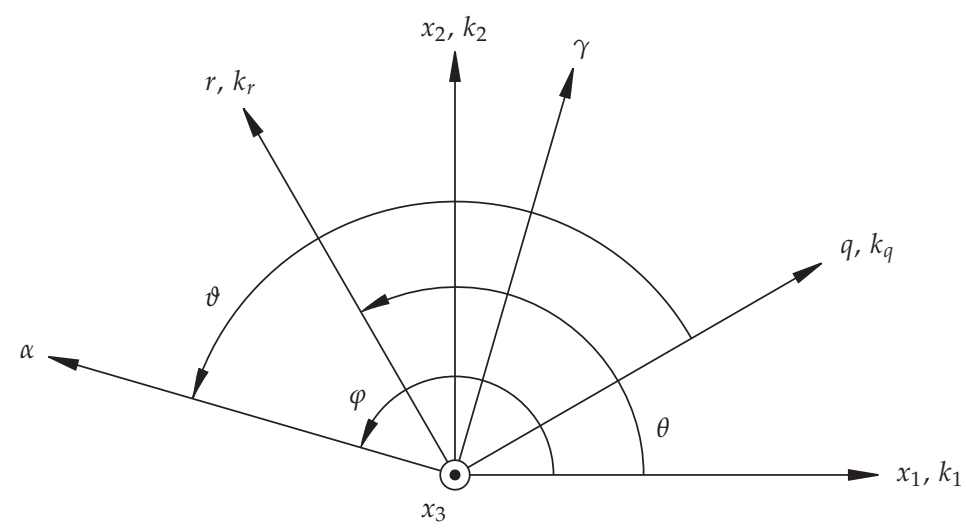

Fig. 5. Definition of the three angles $\varphi, \theta$ and $\vartheta$. 
direction $j$ over an area of rotational symmetry and centred around $(0,0,0)$. In the general case, $\overline{\mathbf{P}}^{10}$ depends on both the angle $\vartheta$ and the wavenumber $\alpha$. However, if the complex amplitudes of the load are independent of $\vartheta$, i.e. if the load is applied with rotational symmetry around the point $(0,0,0)$, the vector $\overline{\mathbf{P}}^{10}$ may be taken outside the integral over $\vartheta$ in Eq. (63), thus reducing computation time in numerical algorithms considerably:

$$
\begin{aligned}
& \mathbf{U}^{10}=\frac{1}{2 \pi} \mathbf{R}(\theta) \int_{0}^{\infty} \widetilde{\mathbf{G}}[\mathbf{R}(\theta)]^{T} \overline{\mathbf{P}}^{10} \alpha \mathrm{d} \alpha, \\
& \widetilde{\mathbf{G}}=\frac{1}{2 \pi} \int_{0}^{2 \pi} \mathbf{R}(\vartheta) \widehat{\overline{\mathbf{G}}}[\mathbf{R}(\vartheta)]^{T} \mathrm{e}^{\mathrm{i} \alpha r \sin \vartheta} \mathrm{d} \vartheta .
\end{aligned}
$$

Examples of the analytical evaluation of axisymmetric loads are given in Subsection 2.6.

Apparently Eq. (64) seems more complicated than the corresponding inverse Fourier transform in Cartesian coordinates given by Eq. (6). However, the integrals over each of the components with respect to $\vartheta$, i.e. the nine integrals involved in the computation of $\widetilde{G}_{k n}(\alpha, r, \omega)$ are identified as Hankel transforms which may be evaluated by means of Bessel functions:

$$
\begin{aligned}
& \frac{1}{2 \pi} \int_{0}^{2 \pi} \mathrm{e}^{\mathrm{i} \alpha r \sin \vartheta} \mathrm{d} \vartheta=\mathrm{J}_{0}(\alpha r), \quad \frac{1}{2 \pi} \int_{0}^{2 \pi} \sin ^{2} \vartheta \mathrm{e}^{\mathrm{i} \alpha r \sin \vartheta} \mathrm{d} \vartheta=\mathrm{J}_{0}(\alpha r)-\frac{1}{\alpha r} \mathrm{~J}_{1}(\alpha r), \\
& \frac{1}{2 \pi} \int_{0}^{2 \pi} \sin \vartheta \mathrm{e}^{\mathrm{i} \alpha r \sin \vartheta} \mathrm{d} \vartheta=\mathrm{i} \mathrm{J}_{1}(\alpha r), \quad \frac{1}{2 \pi} \int_{0}^{2 \pi} \cos ^{2} \vartheta \mathrm{e}^{\mathrm{i} \alpha r \sin \vartheta} \mathrm{d} \vartheta=\frac{1}{\alpha r} \mathrm{~J}_{1}(\alpha r) .
\end{aligned}
$$

Here, $\mathrm{J}_{n}(\alpha r)$ is the Bessel function of the first kind and order $n$. Series expansions of these functions were given by Abramowitz \& Stegun (1972), and routines for their evaluation are available in MATLAB and FORTRAN. Alternatively, the integrals may be given in terms of modified Bessel functions or Hankel functions. Note that the remaining kernels of the integrals in Eq. (65b) are odd functions of $\vartheta$ on the interval $[-\pi ; \pi]$. Therefore these integrals vanish.

Application of the Bessel functions in accordance with Eq. (66) and further taking into account that the Green's function tensor is skew symmetric with $\widehat{\bar{G}}_{12}=\widehat{\bar{G}}_{13}=\widehat{\bar{G}}_{21}=\widehat{\bar{G}}_{31}=0$, see Eq. (55), the components of the integral in Eq. (65b) become

$$
\begin{gathered}
\widetilde{G}_{11}(\alpha, r, \omega)=\left(\mathrm{J}_{0}(\alpha r)-\frac{1}{\alpha r} \mathrm{~J}_{1}(\alpha r)\right) \widehat{\bar{G}}_{11}+\frac{1}{\alpha r} \mathrm{~J}_{1}(\alpha r) \widehat{\bar{G}}_{22}, \\
\widetilde{G}_{22}(\alpha, r, \omega)=\frac{1}{\alpha r} \mathrm{~J}_{1}(\alpha r) \widehat{\bar{G}}_{11}+\left(\mathrm{J}_{0}(\alpha r)-\frac{1}{\alpha r} \mathrm{~J}_{1}(\alpha r)\right) \widehat{\bar{G}}_{22}, \\
\widetilde{G}_{12}(\alpha, r, \omega)=\widetilde{G}_{13}(\alpha, r, \omega)=\widetilde{G}_{21}(\alpha, r, \omega)=\widetilde{G}_{31}(\alpha, r, \omega)=0, \\
\widetilde{G}_{23}(\alpha, r, \omega)=-\widetilde{G}_{32}(\alpha, r, \omega)=\mathrm{iJ}_{1}(\alpha r) \widehat{\bar{G}}_{23}, \quad \widetilde{G}_{33}(\alpha, r, \omega)=\mathrm{J}_{0}(\alpha r) \widehat{\bar{G}}_{33} .
\end{gathered}
$$

Hence, the numerical integration involved in the double inverse Fourier transformation (65a) is reduced to a line integral with respect to $\alpha$. The relations listed in Eq. (67) were established by Auersch (1994). As $\alpha r \rightarrow 0$ the terms $\widetilde{G}_{11}(\alpha, r, \omega)$ and $\widetilde{G}_{22}(\alpha, r, \omega)$ approach the limit

$$
\lim _{\alpha r \rightarrow 0} \widetilde{G}_{11}(\alpha, r, \omega)=\lim _{\alpha r \rightarrow 0} \widetilde{G}_{22}(\alpha, r, \omega)=\frac{\widehat{\bar{G}}_{11}+\widehat{\bar{G}}_{22}}{2} .
$$




\subsection{Analytical evaluation of loads in the Fourier domain}

In order to establish the solution for the displacements in the wavenumber domain, the surface load must first be Fourier transformed over the horizontal Cartesian coordinates. This may be done numerically by application of, for example, an FFT algorithm. However, the computation speed may be improved if the Fourier transformations are carried out analytically. In this subsection, the load spectrum in wavenumber domain is derived for selected surface load distributions.

\subsubsection{A vertical point force on the ground surface}

The load on the surface of the half-space is applied as a vertical point force with the magnitude $P_{0}$ and acting at the origin of the frame of reference. With $\delta(x)$ denoting the Dirac delta function, the amplitude function may be expressed in Cartesian coordinates as

$$
P_{3}^{10}\left(x_{1}, x_{2}, \omega\right)=P_{0} \delta\left(x_{1}\right) \delta\left(x_{2}\right) .
$$

Double Fourier transformation with respect to the horizontal coordinates provides the load spectrum in wavenumber domain:

$$
\bar{P}_{3}^{10}\left(k_{1}, k_{2}, \omega\right)=\int_{-\infty}^{\infty} \int_{-\infty}^{\infty} P_{3}^{10}\left(x_{1}, x_{2}, \omega\right) \mathrm{e}^{-\mathrm{i}\left(k_{1} x_{1}+k_{2} x_{2}\right)} \mathrm{d} x_{1} \mathrm{~d} x_{2}=P_{0} .
$$

Thus, the load simply reduces to a constant in the wavenumber domain. While this load spectrum is very simple, it is not very useful seen in a perspective of numerical computation. A decrease in the kernel of the plane integral with respect to $k_{1}$ and $k_{2}$ is present due to the nature of the Green's function tensor. However, as illustrated in the following subsections, a stronger decay is achieved by distributing the load over a finite area, and a very strong and monotonous decay is observed for a traction applied on the entire ground surface but with diminishing contributions away from the centre point of the loaded area.

\subsubsection{A vertical circular surface load}

The vertical surface load is now applied over a circular area with radius $r_{0}$ and centred at the origin of a cylindrical frame of reference. The load is applied axisymmetrically and in phase with the amplitude function $P_{3}^{10}(r, \omega)$ given as

$$
P_{3}^{10}(r, \omega)=\left\{\begin{array}{cl}
P_{0} /\left(\pi r_{0}^{2}\right) & \text { for } r \leq r_{0} \\
0 & \text { else. }
\end{array}\right.
$$

Double Fourier transformation with respect to the polar coordinates $(r, \theta)$ yields

$$
\bar{P}_{3}^{10}(\alpha, \omega)=\int_{0}^{\infty} \int_{0}^{2 \pi} P_{3}^{10}(r, \omega) \mathrm{e}^{-\mathrm{i} \alpha r \sin \vartheta} \mathrm{d} \vartheta r \mathrm{~d} r=\frac{P_{0}}{\pi r_{0}^{2}} \int_{0}^{r_{0}} 2 \pi \mathrm{J}_{0}(\alpha r) r \mathrm{~d} r=\frac{2 P_{0}}{\alpha r_{0}} \mathrm{~J}_{1}\left(\alpha r_{0}\right) .
$$

Here, $\alpha$ is the radial wavenumber and $\vartheta=\pi / 2+\varphi-\theta$ is the angle between the wavenumber and the radius vectors in polar coordinates $(\alpha, \varphi)$ and $(r, \theta)$, respectively. As discussed above, $\alpha r \sin \vartheta$ is the scalar product between the vectors with lengths $\alpha$ and $r$, respectively.

Clearly, the load spectrum decays rapidly with $\alpha$ which is present both in the denominator of the fraction and in the argument of the Bessel function of the first kind and order 1 . The decay rate increases if the load is distributed over a large area in spatial domain, i.e. if $r_{0}$ is large. However, at $\alpha=0$, the spectrum has a strong singularity. 


\subsubsection{A vertical "bell-shaped" surface load}

Finally, applying a Gaussian distribution of $P_{3}^{10}(r, \omega)$ leads to a "bell-shaped" load on the surface of the half-space. In polar coordinates, a vertical load of this kind is expressed as

$$
P_{3}^{10}(r, \omega)=\frac{P_{0}}{4 \pi r_{0}^{2}} \mathrm{e}^{-\left(\frac{r}{2 r_{0}}\right)^{2}} .
$$

A small value of $r_{0}$ (the standard deviation) defines a nearly concentrated force. In the limit as $r_{0} \rightarrow 0$, the "bell-shaped" load approaches the delta spike discussed in the first example. Double Fourier transformation of $P_{3}^{10}(r, \omega)$ with respect to the polar coordinates $(r, \theta)$ yields

$$
\bar{P}_{3}^{10}(\alpha, \omega)=\int_{0}^{\infty} \int_{0}^{2 \pi} P_{3}^{10}(r, \omega) \mathrm{e}^{-\mathrm{i} \alpha r \sin \vartheta} \mathrm{d} \vartheta r \mathrm{~d} r=P_{0} \mathrm{e}^{-\alpha^{2} r_{0}^{2}},
$$

where the usual interpretation of $\alpha r \sin \vartheta$ as a scalar product between two vectors applies. The definition of the angle $\vartheta$ is given in Fig. 5 .

Hence, in the spatial domain, the "bell-shaped" load is subject to an exponential decay with increasing radius $r$ and decreasing standard deviation $r_{0}$. In the wavenumber domain, there is an exponential decay of $\bar{P}_{3}^{10}(\alpha, \omega)$ with respect to $\alpha$ as well as $r_{0}$ squared. This results in a load that is adequate for numerical evaluation of the inverse Fourier transform of the response in wavenumber domain. A further discussion can be found in the next section.

\section{Dynamic stiffness of rigid surface footings of arbitrary shape}

Independent of its shape, a rigid footing has three translational and three rotational degrees of freedom as shown in Fig. 6. In the frequency domain, these are related to the corresponding forces and moments via the impedance matrix $\mathbf{Z}(\omega)$,

$$
\begin{gathered}
\mathbf{Z}(\omega) \mathbf{V}(\omega)=\mathbf{F}(\omega), \\
\mathbf{V}(\omega)\left[\begin{array}{llllll}
V_{1} & V_{2} & V_{3} & \Theta_{1} & \Theta_{2} & \Theta_{3}
\end{array}\right]^{T}, \\
\mathbf{F}(\omega)\left[\begin{array}{llllll}
Q_{1} & Q_{2} & Q_{3} & M_{1} & M_{2} & M_{3}
\end{array}\right]^{T} .
\end{gathered}
$$

In the most general case, the impedance matrix $\mathbf{Z}(\omega)$ is full, i.e. all the rigid-body motions of the footing are interrelated. However, in the present case the footing rests on the surface of a horizontally layered stratum. Further, assuming that the stress resultants act at the

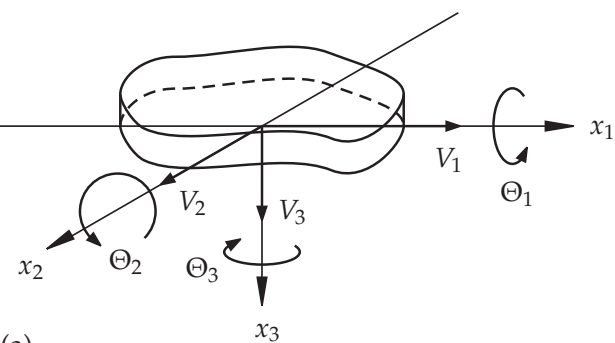

(a)

Fig. 6. Degrees of freedom for a rigid surface footing in the frequency domain:

(a) displacements and rotations, and (b) forces and moments. 
centre of the soil-foundation interface, the torsional and vertical displacements are completely decoupled from the remaining degrees of freedom. Thus, the impedance matrix simplifies to

$$
\mathbf{Z}(\omega)=\left[\begin{array}{llllll}
Z_{11} & Z_{12} & 0 & Z_{14} & Z_{15} & 0 \\
Z_{12} & Z_{22} & 0 & Z_{24} & Z_{25} & 0 \\
0 & 0 & Z_{33} & 0 & 0 & 0 \\
Z_{14} & Z_{24} & 0 & Z_{44} & Z_{45} & 0 \\
Z_{15} & Z_{55} & 0 & Z_{45} & Z_{55} & 0 \\
0 & 0 & 0 & 0 & 0 & Z_{66}
\end{array}\right] .
$$

A further simplification of $\mathbf{Z}(\omega)$ is obtained if the moment of inertia around a given horizontal axis is invariant to a rotation of the footing around the $z$-axis. This is the case for the gravitation foundations that are typically utilised for wind turbines, i.e. circular, square, hexagonal and octagonal footings. With reference to Fig. 7, the moments of inertia are $I_{x_{1}}=I_{x_{2}}=I_{\xi}=I_{\zeta}$, where $\zeta$ is an arbitrary horizontal axis. As a result of this, $Z_{11}=Z_{22}, Z_{44}=Z_{55}$ and $Z_{15}=$ $-Z_{24}$, and the coupling between sliding in the $x_{1}$-direction and rocking in the $x_{2}$-direction (and vice versa) vanishes, i.e.

(a)

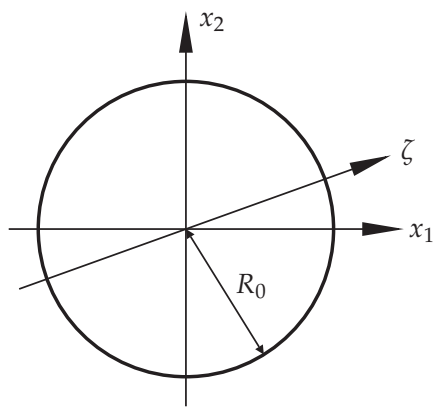

(c)

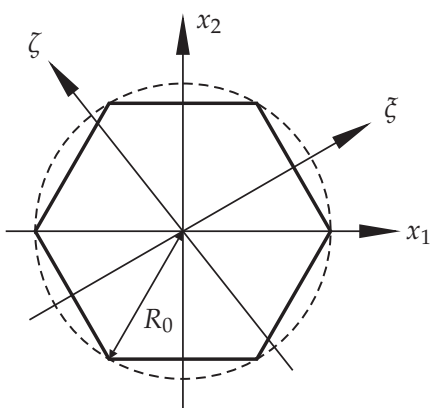

(b)

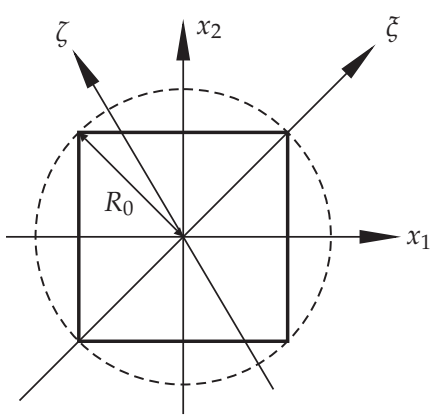

(d)

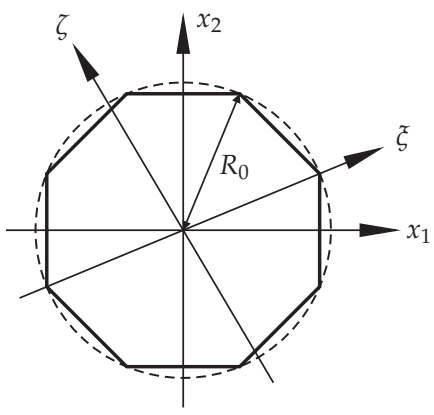

Fig. 7. Definition of axes for different geometries of a footing: (a) circular, (b) square, (c) hexagonal, and (d) octagonal footing. The horizontal plane is considered, and all the footings have the same characteristic length, $R_{0}$. 


$$
\mathbf{Z}(\omega)=\left[\begin{array}{clllll}
Z_{11} & 0 & 0 & 0 & -Z_{24} & 0 \\
0 & Z_{22} & 0 & Z_{24} & 0 & 0 \\
0 & 0 & Z_{33} & 0 & 0 & 0 \\
0 & Z_{24} & 0 & Z_{44} & 0 & 0 \\
-Z_{24} & 0 & 0 & 0 & Z_{55} & 0 \\
0 & 0 & 0 & 0 & 0 & Z_{66}
\end{array}\right]
$$

\subsection{Evaluation of the dynamic stiffness based on the flexibility of a layered ground}

In order to compute the nonzero components of the impedance matrix $\mathbf{Z}(\omega)$, the distribution of the contact stresses at the interface between the footing and the ground due to given rigid body displacements has to be determined. However, Eq. (63) provides the displacement field for a known stress distribution. Generally this implies that the problem takes the form of an integral equation. For the particular case of a circular footing on a homogeneous half-space, Krenk \& Schmidt (1981) derived a closed-form solution for the vertical impedance. Yong et al. (1997) proposed that the total contact stress be decomposed into a number of simple distributions obtained by a Fourier series with respect to the azimuthal angle and a polynomial in the radial direction, e.g.

$$
P_{r}^{10}(r, \vartheta, \omega)=\sum_{m=1}^{M} \sum_{n=1}^{N} a_{m n} r^{n} \cos (m \vartheta)
$$

for the component in the $r$-direction and a symmetric contact stress distribution. Similar expressions were given for the components in the $q$ - (or $\vartheta_{-}$) and $x_{3}$-direction and for the antisymmetric case. The response to each of the contact stress distributions can be computed, and the coefficients $a_{m n}$ are determined so that the prescribed rigid body displacements are obtained.

However, for arbitrary shapes of the footing it may be difficult to follow this idea. Hence, in this study a different approach is taken which has the following steps:

1. The displacement corresponding to each rigid body mode is prescribed at $N$ points distributed uniformly at the interface between the footing and the ground.

2. The Green's function matrix is evaluated in the wavenumber domain along the $\alpha$-axis, and Eq. (65b) is evaluated by application of Eq. (67).

3. The wavenumber spectrum for a simple distributed load with unit magnitude and rotational symmetry around a point on the ground surface is computed. As discussed in Example 2.6.3, a "bell-shaped" load based on a double Gaussian distribution has the advantage that the wavenumber spectrum is a monotonic decreasing function of $\alpha$.

4. The response at point $n$ to a load centred at point $m$ is calculated for all combinations of $n, m=1,2, \ldots, N$. This provides a flexibility matrix for the footing.

5. The unknown magnitudes of the loads applied around each of the points are computed. Integration over the contact area provides the impedance.

The discretization of the soil-foundation interface into $N$ points and the employment of the "bell-shaped" load distribution are visualized in Fig. 8 for a hexagonal footing.

In particular, if the surface traction vector in the wavenumber-frequency domain takes the form $\bar{P}_{i}^{10}\left(k_{1}, k_{2}, \omega\right)=\bar{D}\left(k_{1}, k_{2}\right) \widetilde{P}_{i}(\omega), i=1,2,3$, where $\bar{D}\left(k_{1}, k_{2}\right)$ is a stress distribution with unit magnitude and $\widetilde{P}_{i}(\omega)$ is an amplitude, Eq. (65a) may be computed as 


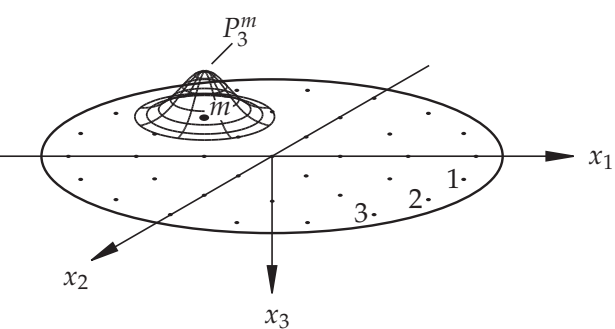

(a)

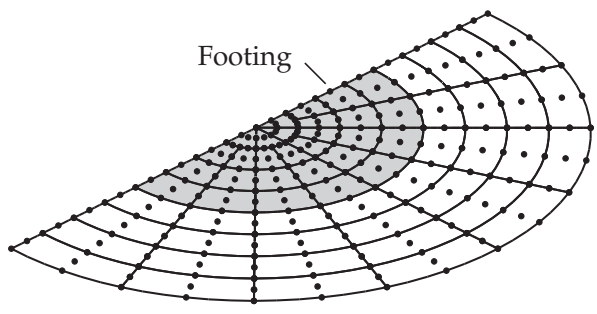

(b)

Fig. 8. Discretization of the soil-foundation interface for a hexagonal footing. The vertical component of the "bell-shaped" load at point $m$ is shown.

$$
\mathbf{U}^{10}=\mathbf{R}(\theta) \widehat{\mathbf{G}}[\mathbf{R}(\theta)]^{T} \widetilde{\mathbf{P}}, \quad \widehat{\mathbf{G}}=\widehat{\mathbf{G}}(r, \omega)=\frac{1}{2 \pi} \int_{0}^{\infty} \widetilde{\mathbf{G}} \widehat{\bar{D}} \alpha \mathrm{d} \alpha .
$$

Here it is noted that $\widehat{\bar{D}}=\widehat{\bar{D}}(\alpha)=\bar{D}(0, \alpha)$, since an axisymmetric distribution is assumed. The choice of contact stress distribution and various discretization aspects are discussed below. Alternatively, a boundary element model based on the Green's function for the layered half-space may be employed. However, this involves some additional work, since the Green's function for traction has to be evaluated.

\subsection{Discretization considerations}

In order to achieve an accurate and efficient computation of the impedance matrix for a footing with the present method, a number of issues need consideration:

1. Equation (76) has to be evaluated numerically. This requires a computation of $\widehat{\overline{\mathbf{G}}}(\alpha, \omega)$ for a number of discrete wavenumbers. All peaks in the wavenumber spectrum must be represented well, demanding a fine discretization in the low wavenumber range-in particular for a half-space with little material damping.

2. No significant contributions may exist from the products $\widehat{\bar{D}}(\alpha) \widehat{\bar{G}}_{i j}(\alpha, \omega), i, j=1,2,3$, for wavenumbers beyond the truncation point in the numerical evaluation of the integral in Eq. (76).

3. Enough points should be employed at the soil-structure interface in order to provide a good approximation of the contact stress distribution.

Concerning item 1 it is of paramount importance to determine the wavenumber below which the wavenumber spectrum may have narrow-banded peaks. Here use can be made of the fact that the longest wave present in a homogeneous half-space is the Rayleigh wave. An approximate upper limit for the Rayleigh wavenumber is provided by the inequality $\alpha_{R}=$ $\omega / c_{R}<1.2 \omega / c_{S}$ for $v \in[0 ; 0.5]$. For a stratum with $J$ layers overlaying a homogeneous half-space, the idea is now to determine the quantity

$$
\alpha_{1}=2 \omega / \min \left\{c_{S}^{1}, c_{S}^{2}, \ldots, c_{S}^{J+1}\right\} .
$$

where index $J+1$ refers to the underlying homogeneous half-space. In a stratum, waves with wavenumbers higher than $\alpha_{1}$ are generally subject to strong material dissipation since they arise from $\mathrm{P}$ - or $\mathrm{S}$-waves being reflected multiple times at the interfaces between layers. Only 
if the loss factor is $\eta^{j}=0$ for all layers, undamped Love waves may exist; but this situation is not likely to appear in real soils where typical values are $\eta^{j} \approx 0.01$ to 0.1 .

Concerning item 2 it has been found by numerical experiments that the integral of Eq. (76) may be truncated beyond the wavenumber $\alpha_{2}$ determined as

$$
\alpha_{2}=\max \left\{5 \alpha_{1}, 20 \alpha_{0}\right\}, \quad \alpha_{0}=2 \pi / R_{0} .
$$

Here $R_{0}$ is a characteristic length of the foundation, e.g. the diameter of a circular footing. For strata with $\eta^{j}>0.01$ for all layers it has been found that accurate results are typically obtained by Simpson integration with 2000 points in the wavenumber range $\alpha \in\left[0 ; \alpha_{1}\right]$ and 500 points in the range $\alpha \in\left[\alpha_{1} ; \alpha_{2}\right]$. As discussed above, the numerical evaluation of the integral in the range $\alpha \in\left[\alpha_{1} ; \alpha_{2}\right]$ is particularly efficient for the "bell-shaped" load distribution, since $\widehat{\bar{D}}(\alpha) \widehat{\bar{G}}_{i j}(\alpha, \omega), i, j=1,2,3$, are all monotone functions beyond $\alpha_{1}$.

Finally, concerning item $3, \mathbf{U}^{10}$ must be evaluated for all combinations of receiver and source points, which involves a high number of computations if $\widehat{\mathbf{G}}(r, \omega)$ is to be evaluated directly for each value of $r$. Instead, an alternative approach is suggested. Firstly, $\widehat{\mathbf{G}}(r, \omega)$ is determined at a number of points on the $r$-axis from $r=0$ to $r=2 R_{0}$. Subsequently $\mathbf{U}^{10}$ is found by Eq. (76) using linear interpolation of $\widehat{\mathbf{G}}(r, \omega)$. It has been found that 250 points in this discretization provides a fast solution of satisfactory accuracy.

\section{Consistent lumped-parameter models for wind-turbine foundations}

Dynamic soil-structure interaction of wind turbines may be analysed by the finite-element method (FEM), the boundary-element method (BEM) or the domain-transformation method (DTM) described in the previous sections. These methods are highly adaptable and may be applied to the analysis of wave-propagation problems involving stratified soil, embedded foundations and inclusions or inhomogeneities in the ground. However, this comes at the cost of great computation times, in particular in the case of time-domain analysis of transient structural response over large periods of time. Thus, rigorous numerical models based on the FEM, the BEM, or the DTM, are not useful for real-time simulations or parametric studies in situations where only the structural response is of interest.

Alternatively, soil-structure interaction may be analysed by experimental methods. However, the models and equipment required for such analyses are expensive and this approach is not useful in a predesign phase. Hence, the need arises for a computationally efficient model which accounts for the interaction of a wind turbine foundation with the surrounding/underlying soil. A fairly general solution is the so-called lumped-parameter model, the development of which has been reported by Wolf (1991a), Wolf \& Paronesso (1991), Wolf (1991b), Wolf \& Paronesso (1992), Wolf (1994), Wolf (1997), Wu \& Lee (2002), and Wu \& Lee (2004). The present section is, to a great extent, based on this work.

The basic concept of a lumped-parameter model is to represent the original problem by a simple mechanical system consisting of a few so-called discrete elements, i.e. springs, dashpots and point masses which are easily implemented in standard finite-element models or aero-elastic codes for wind turbines. This is illustrated in Fig. 1 for a surface footing on a layered ground. The computational model consists of two parts: a model of the structure (e.g. a finite-element model) and a lumped-parameter model (LPM) of the foundation and the subsoil. The formulation of the model has three steps:

1. A rigorous frequency-domain model is applied for the foundation (in this case a footing on a soil stratum) and the frequency response is evaluated at a number of discrete frequencies. 
2. A lumped-parameter model providing approximately the same frequency response is calibrated to the results of the rigorous model.

3. The structure itself (in this case the wind turbine) is represented by a finite-element model (or similar) and soil-structure interaction is accounted for by a coupling with the LPM of the foundation and subsoil.

Whereas the application of rigorous models like the BEM or DTM is often restricted to the analysis in the frequency domain - at least for any practical purposes-the LPM may be applied in the frequency domain as well as the time domain. This is ideal for problems involving linear response in the ground and nonlinear behaviour of a structure, which may typically be the situation for a wind turbine operating in the serviceability limit state (SLS).

It should be noted that the geometrical damping present in the original wave-propagation problem is represented as material damping in the discrete-element model. Thus, no distinction is made between material and geometrical dissipation in the final lumped-parameter model — they both contribute to the same parameters, i.e. damping coefficients.

Generally, if only few discrete elements are included in the lumped-parameter model, it can only reproduce a simple frequency response, i.e. a response with no resonance peaks. This is useful for rigid footings on homogeneous soil. However, inhomogeneous or flexible structures and stratified soil have a frequency response that can only be described by a lumped-parameter model with several discrete elements resulting in the presence of internal degrees of freedom. When the number of internal degrees of freedom is increased, so is the computation time. However, so is the quality of the fit to the original frequency response. This is the idea of the so-called consistent lumped-parameter model which is presented in this section.

\subsection{Approximation of soil-foundation interaction by a rational filter}

The relationship between a generalised force resultant, $f(t)$, acting at the foundation-soil interface and the corresponding generalised displacement component, $v(t)$, can be approximated by a differential equation in the form:

$$
\sum_{i=0}^{k} A_{i} \frac{\mathrm{d}^{i} v(t)}{\mathrm{d} t^{i}}=\sum_{j=0}^{l} B_{j} \frac{\mathrm{d}^{j} f(t)}{\mathrm{d} t^{j}} .
$$

Here, $A_{i}, i=1,2, \ldots, k$, and $B_{j}, j=1,2, \ldots, l$, are real coefficients found by curve fitting to the exact analytical solution or the results obtained by some numerical method or measurements. The rational approximation (79) suggests a model, in which higher-order temporal derivatives of both the forces and the displacements occur. This is undesired from a computational point of view. However, a much more elegant model only involving the zeroth, the first and the second temporal derivatives may be achieved by a rearrangement of the differential operators. This operation is simple to carry out in the frequency domain; hence, the first step in the formulation of a rational approximation is a Fourier transformation of Eq. (79), which provides:

$$
\begin{gathered}
\sum_{i=0}^{k} A_{i}(\mathrm{i} \omega)^{i} V(\omega)=\sum_{j=0}^{l} B_{j}(\mathrm{i} \omega)^{j} Q(\omega) \Rightarrow \\
Q(\omega)=\widehat{Z}(\mathrm{i} \omega) V(\omega), \quad \widehat{Z}(\mathrm{i} \omega)=\frac{\sum_{i=0}^{k} A_{i}(\mathrm{i} \omega)^{i}}{\sum_{j=0}^{l} B_{j}(\mathrm{i} \omega)^{j}},
\end{gathered}
$$


where $V(\omega)$ and $F(\omega)$ denote the complex amplitudes of the generalized displacements and forces, respectively. It is noted that in Eq. (80) it has been assumed that the reaction force $F(\omega)$ stems from the response to a single displacement degree of freedom. This is generally not the case. For example, as discussed in Section 3, there is a coupling between the rocking moment-rotation and the horizontal force-translation of a rigid footing. However, the model (80) is easily generalised to account for such behaviour by an extension in the form $F_{i}(\omega)=\widehat{Z}_{i j}(\mathrm{i} \omega) V_{j}(\omega)$, where summation is carried out over index $j$ equal to the degrees of freedom contributing to the response. Each of the complex stiffness terms, $\widehat{Z}_{i j}(\mathrm{i} \omega)$, is given by a polynomial fraction as illustrated by Eq. (80) for $\widehat{Z}(\mathrm{i} \omega)$. This forms the basis for the derivation of so-called consistent lumped-parameter models.

\subsection{Polynomial-fraction form of a rational filter}

In the frequency domain, the dynamic stiffness related to a degree of freedom, or to the interaction between two degrees of freedom, $i$ and $j$, is given by $\widetilde{Z}_{i j}\left(a_{0}\right)=Z_{i j}^{0} S_{i j}\left(a_{0}\right)$ (no sum on $i, j)$. Here, $Z_{i j}^{0}=Z_{i j}(0)$ denotes the static stiffness related to the interaction of the two degrees of freedom, and $a_{0}=\omega R_{0} / c_{0}$ is a dimensionless frequency with $R_{0}$ and $c_{0}$ denoting a characteristic length and wave velocity, respectively. For example, for a circular footing with the radius $R_{0}$ on an elastic half-space with the S-wave velocity $c_{S}, a_{0}=\omega R_{0} / c_{S}$ may be chosen. With the given normalisation of the frequency it is noted that $\widetilde{Z}_{i j}\left(a_{0}\right)=$ $Z_{i j}\left(c_{0} a_{0} / R_{0}\right)=Z_{i j}(\omega)$.

For simplicity, any indices indicating the degrees of freedom in question are omitted in the following subsections, e.g. $\widetilde{Z}\left(a_{0}\right) \sim \widetilde{Z}_{i j}\left(a_{0}\right)$. The frequency-dependent stiffness coefficient $S\left(a_{0}\right)$ for a given degree of freedom is then decomposed into a singular part, $S_{S}\left(a_{0}\right)$, and a regular part, $S_{r}\left(a_{0}\right)$, i.e.

$$
\widetilde{Z}\left(a_{0}\right)=Z^{0} S\left(a_{0}\right), \quad S\left(a_{0}\right)=S_{s}\left(a_{0}\right)+S_{r}\left(a_{0}\right),
$$

where $Z^{0}$ is the static stiffness, and the singular part has the form

$$
S_{s}\left(a_{0}\right)=k^{\infty}+\mathrm{i} a_{0} c^{\infty} .
$$

In this expression, $k^{\infty}$ and $c^{\infty}$ are two real-valued constants which are selected so that $Z^{0} S_{s}\left(a_{0}\right)$ provides the entire stiffness in the high-frequency limit $a_{0} \rightarrow \infty$. Typically, the stiffness term $Z^{0} k^{\infty}$ vanishes and the complex stiffness in the high-frequency range becomes a pure mechanical impedance, i.e. $S_{s}\left(a_{0}\right)=\mathrm{i} a_{0} c^{\infty}$. This is demonstrated in Section 5 for a two different types of wind turbine foundations interacting with soil.

The regular part $S_{r}\left(a_{0}\right)$ accounts for the remaining part of the stiffness. Generally, a closed-form solution for $S_{r}\left(a_{0}\right)$ is unavailable. Hence, the regular part of the complex stiffness is usually obtained by fitting of a rational filter to the results obtained with a numerical or semi-analytical model using, for example, the finite-element method (FEM), the boundary-element method (BEM) or the domain-transformation method (DTM). Examples are given in Section 5 for wind turbine foundations analysed by each of these methods.

Whether an analytical or a numerical solution is established, the output of a frequency-domain analysis is the complex dynamic stiffness $\widetilde{Z}\left(a_{0}\right)$. This is taken as the "target solution", and the regular part of the stiffness coefficient is found as $S_{r}\left(a_{0}\right)=\widetilde{Z}\left(a_{0}\right) / Z^{0}-S_{s}\left(a_{0}\right)$. A rational approximation, or filter, is now introduced in the form

$$
S_{r}\left(a_{0}\right) \approx \widehat{S}_{r}\left(\mathrm{i} a_{0}\right)=\frac{P\left(\mathrm{i} a_{0}\right)}{Q\left(\mathrm{i} a_{0}\right)}=\frac{p_{0}+p_{1}\left(\mathrm{i} a_{0}\right)+p_{2}\left(\mathrm{i} a_{0}\right)^{2}+\ldots+p_{N}\left(\mathrm{i} a_{0}\right)^{N}}{q_{0}+q_{1}\left(\mathrm{i} a_{0}\right)+q_{2}\left(\mathrm{i} a_{0}\right)^{2}+\ldots+q_{M}\left(\mathrm{i} a_{0}\right)^{M}} .
$$


The orders, $N$ and $M$, and the coefficients, $p_{n}(n=0,1, \ldots, N)$ and $q_{m}(m=0,1, \ldots, M)$, of the numerator and denominator polynomials $P\left(\mathrm{i} a_{0}\right)$ and $Q\left(\mathrm{i} a_{0}\right)$ are chosen according to the following criteria:

1. To obtain a unique definition of the filter, one of the coefficients in either $P\left(\mathrm{i} a_{0}\right)$ or $Q\left(\mathrm{i} a_{0}\right)$ has to be given a fixed value. For convenience, $q_{0}=1$ is chosen.

2. Since part of the static stiffness is already represented by $S_{S}(0)=k^{\infty}$, this part of the stiffness should not be provided by $S_{r}\left(a_{0}\right)$ as well. Therefore, $p_{0} / q_{0}=p_{0}=1-k^{\infty}$.

3. In the high-frequency limit, $S\left(a_{0}\right)=S_{S}\left(a_{0}\right)$. Thus, the regular part must satisfy the condition that $\widehat{S}_{r}\left(\mathrm{i} a_{0}\right) \rightarrow 0$ for $a_{0} \rightarrow \infty$. Hence, $N<M$, i.e. the numerator polynomial $P\left(\mathrm{i} a_{0}\right)$ is at least one order lower than the denominator polynomial, $Q\left(\mathrm{i} a_{0}\right)$.

Based on these criteria, Eq. (84) may advantageously be reformulated as

$$
S_{r}\left(a_{0}\right) \approx \widehat{S}_{r}\left(\mathrm{i} a_{0}\right)=\frac{P\left(\mathrm{i} a_{0}\right)}{Q\left(\mathrm{i} a_{0}\right)}=\frac{1-k^{\infty}+p_{1}\left(\mathrm{i} a_{0}\right)+p_{2}\left(\mathrm{i} a_{0}\right)^{2}+\ldots+p_{M-1}\left(\mathrm{i} a_{0}\right)^{M-1}}{1+q_{1}\left(\mathrm{i} a_{0}\right)+q_{2}\left(\mathrm{i} a_{0}\right)^{2}+\ldots+q_{M}\left(\mathrm{i} a_{0}\right)^{M}} .
$$

Evidently, the polynomial coefficients in Eq. (84) must provide a physically meaningful filter. By a comparison with Eqs. (79) and (80) it follows that $p_{n}(n=1,2, \ldots, M-1)$ and $q_{j}(m=$ $1,2, \ldots M)$ must all be real. Furthermore, no poles should appear along the positive real axis as this will lead to an unstable solution in the time domain. This issue is discussed below.

The total approximation of $S\left(a_{0}\right)$ is found by an addition of Eqs. (82) and (84) as stated in Eq. (81). The approximation of $S\left(a_{0}\right)$ has two important characteristics:

- It is exact in the static limit, since $S\left(a_{0}\right) \approx \widehat{S}\left(\mathrm{i} a_{0}\right)+S_{S}\left(a_{0}\right) \rightarrow 1$ for $a_{0} \rightarrow 0$.

- It is exact in the high-frequency limit. Here, $S\left(a_{0}\right) \rightarrow S_{s}\left(a_{0}\right)$ for $a_{0} \rightarrow \infty$, because $\widehat{S}_{r}\left(\mathrm{i} a_{0}\right) \rightarrow$ 0 for $a_{0} \rightarrow \infty$.

Hence, the approximation is double-asymptotic. For intermediate frequencies, the quality of the fit depends on the order of the rational filter and the nature of the physical problem. Thus, in some situations a low-order filter may provide a very good fit to the exact solution, whereas other problems may require a high-order filter to ensure an adequate match-even over a short range of frequencies. As discussed in the examples given below in Section 5, a filter order of $M=4$ will typically provide satisfactory results for a footing on a homogeneous half-space. However, for flexible, embedded foundations and layered soil, a higher order of the filter may be necessary-even in the low-frequency range relevant to dynamic response of wind turbines.

\subsection{Partial-fraction form of a rational filter}

Whereas the polynomial-fraction form is well-suited for curve fitting to measured or computed responses, it provides little insight into the physics of the problem. To a limited extent, such information is gained by a recasting of Eq. (84) into partial-fraction form,

$$
\widehat{S}_{r}\left(\mathrm{i} a_{0}\right)=\sum_{m=1}^{M} \frac{R_{m}}{\mathrm{i} a_{0}-s_{m}},
$$

where $s_{m}, m=1,2, \ldots, M$, are the poles of $\widehat{S}_{r}\left(\mathrm{i} a_{0}\right)$ (i.e. the roots of $Q\left(\mathrm{i} a_{0}\right)$ ), and $R_{j}$ are the corresponding residues. The conversion of the original polynomial-fraction form into the partial-fraction expansion form may be carried out in MATLAB with the built-in function residue. 
The poles $s_{m}$ are generally complex. However, as discussed above, the coefficients $q_{m}$ must be real in order to provide a rational approximation that is physically meaningful in the time domain. To ensure this, any complex poles, $s_{m}$, and the corresponding residues, $R_{m}$, must appear as conjugate pairs. When two such terms are added together, a second-order term with real coefficients appears. Thus, with $N$ conjugate pairs, Eq. (85) can be rewritten as

$$
\widehat{S}_{r}\left(\mathrm{i} a_{0}\right)=\sum_{n=1}^{N} \frac{\beta_{0 n}+\beta_{1 n} \mathrm{i} a_{0}}{\alpha_{0 n}+\alpha_{1 n} \mathrm{i} a_{0}+\left(\mathrm{i} a_{0}\right)^{2}}+\sum_{n=N+1}^{M-N} \frac{R_{n}}{\mathrm{i} a_{0}-s_{n}}, \quad 2 N \leq M .
$$

The coefficients $\alpha_{0 n}, \alpha_{1 n}, \beta_{0 n}$ and $\beta_{1 n}, n=N+1, N+2, \ldots, M-N$, are given by

$$
\alpha_{0 n}=\left\{s_{n}^{\Re}\right\}^{2}+\left\{s_{n}^{\Im}\right\}^{2}, \quad \alpha_{1 n}=-2 s_{n}^{\Re}, \quad \beta_{0 n}=-2\left(R_{n}^{\Re} s_{n}^{\Re}+R_{n}^{\Im} s_{n}^{\Im}\right), \quad \beta_{1 n}=2 R_{n}^{\Re},
$$

where $s_{n}^{\Re}=\Re\left(s_{n}\right)$ and $s_{n}^{\Im}=\Im\left(s_{n}\right)$ are the real and imaginary parts of the complex conjugate poles, respectively. Similarly, the real and imaginary parts of the complex conjugate residues are denoted by $R_{n}^{\Re}=\Re\left(R_{n}\right)$ and $R_{n}^{\Im}=\Im\left(R_{n}\right)$, respectively.

By adding the singular term in Eq. (82) to the expression in Eq. (85), the total approximation of the dynamic stiffness coefficient $S\left(a_{0}\right)$ can be written as

$$
\widehat{S}\left(\mathrm{i} a_{0}\right)=k^{\infty}+\mathrm{i} a_{0} c^{\infty}+\sum_{n=1}^{N} \frac{\beta_{0 n}+\beta_{1 n} \mathrm{i} a_{0}}{\alpha_{0 n}+\alpha_{1 n} \mathrm{i} a_{0}+\left(\mathrm{i} a_{0}\right)^{2}}+\sum_{n=N+1}^{M-N} \frac{R_{n}}{\mathrm{i} a_{0}-s_{n}} .
$$

The total approximation of the dynamic stiffness in Eq. (88) consists of three characteristic types of terms, namely a constant/linear term, $M-2 N$ first-order terms and $N$ second-order terms. These terms are given as:

$$
\begin{aligned}
\text { Constant/linear term: } & k^{\infty}+\mathrm{i} a_{0} c^{\infty} \\
\text { First-order term: } & \frac{R}{\mathrm{i} a_{0}-s} \\
\text { Second-order term: } & \frac{\beta_{1} \mathrm{i} a_{0}+\beta_{0}}{\alpha_{0}+\alpha_{1} \mathrm{i} a_{0}+\left(\mathrm{i} a_{0}\right)^{2}} .
\end{aligned}
$$

\subsection{Physical interpretation of a rational filter}

Now, each term in Eq. (89) may be identified as the frequency-response function for a simple mechanical system consisting of springs, dashpots and point masses. Physically, the summation of terms (88) may then be interpreted as a parallel coupling of $M-N+1$ of these so-called discrete-element models, and the resulting lumped-parameter model provides a frequency-response function similar to that of the original continuous system. In the subsections below, the calibration of the discrete-element models is discussed, and the physical interpretation of each kind of term in Eq. (89) is described in detail.

\subsubsection{Constant/linear term}

The constant/linear term given by Eq. (89a) consists of two known parameters, $k^{\infty}$ and $c^{\infty}$, that represent the singular part of the dynamic stiffness. The discrete-element model for the constant/linear term is shown in Fig. 9.

The equilibrium formulation of Node 0 (for harmonic loading) is as follows:

$$
\kappa U_{0}(\omega)+\mathrm{i} \omega \gamma \frac{R_{0}}{c_{0}} U_{0}(\omega)=P_{0}(\omega)
$$




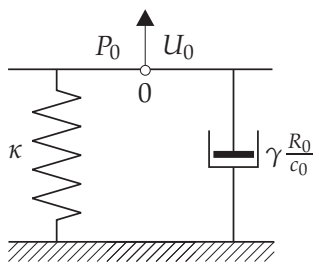

Fig. 9. The discrete-element model for the constant/linear term.

Recalling that the dimensionless frequency is introduced as $a_{0}=\omega R_{0} / c_{0}$, the equilibrium formulation in Eq. (90) results in a force-displacement relation given by

$$
P_{0}\left(a_{0}\right)=\left(\kappa+\mathrm{i} a_{0} \gamma\right) U_{0}\left(a_{0}\right) .
$$

By a comparison of Eqs. (89a) and (91) it becomes evident that the non-dimensional coefficients $\kappa$ and $\gamma$ are equal to $k^{\infty}$ and $c^{\infty}$, respectively.

\subsubsection{First-order terms with a single internal degree of freedom}

The first-order term given by Equation ( $89 \mathrm{~b}$ ) has two parameters, $R$ and $s$. The layout of the discrete-element model is shown in Fig. 10a. The model is constructed by a spring $(-\kappa)$ in parallel with another spring $(\kappa)$ and dashpot $\left(\gamma \frac{R_{0}}{c_{0}}\right)$ in series. The serial connection between the spring $(\kappa)$ and the dashpot $\left(\gamma \frac{R_{0}}{c_{0}}\right)$ results in an internal node (internal degree of freedom). The equilibrium formulations for Nodes 0 and 1 (for harmonic loading) are as follows:

$$
\begin{array}{ll}
\text { Node 0 : } & \kappa\left(U_{0}(\omega)-U_{1}(\omega)\right)-\kappa U_{0}(\omega)=P_{0}(\omega) \\
\text { Node 1: } & \kappa\left(U_{1}(\omega)-U_{0}(\omega)\right)+i \omega \gamma \frac{R_{0}}{c_{0}} U_{1}(\omega)=0 .
\end{array}
$$

After elimination of $U_{1}(\omega)$ in Eqs. (92a) and (92b), it becomes clear that the force-displacement relation of the first-order model is given as

$$
P_{0}\left(a_{0}\right)=\frac{-\frac{\kappa^{2}}{\gamma}}{\mathrm{i} a_{0}+\frac{\kappa}{\gamma}} U_{0}\left(a_{0}\right) .
$$

By comparing Eqs. (89b) and (93), $\kappa$ and $\gamma$ are identified as

$$
\kappa=\frac{R}{S}, \quad \gamma=-\frac{R}{S^{2}} .
$$

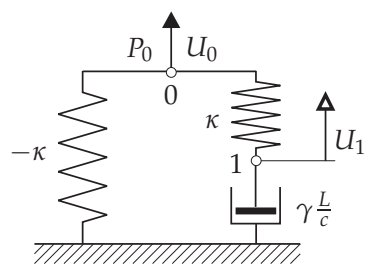

(a)

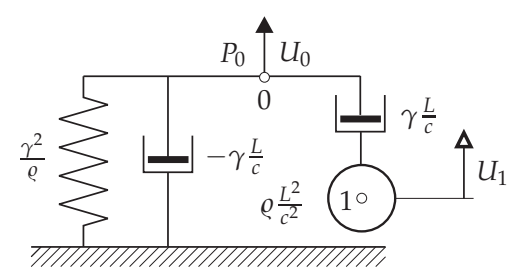

(b)

Fig. 10. The discrete-element model for the first-order term: (a) Spring-dashpot model; (b) monkey-tail model. 
It should be noted that the first-order term could also be represented by a so-called "monkey-tail" model, see Fig. 10b. This turns out to be advantageous in situations where $\kappa$ and $\gamma$ in Eq. (94) are negative, which may be the case when $R$ is positive ( $s$ is negative). To avoid negative coefficients of springs and dashpots, the monkey-tail model is applied, and the resulting coefficients are positive. By inspecting the equilibrium formulations for Nodes 0 and 1 , see Fig. 10b, the coefficients can be identified as

$$
\gamma=\frac{R}{s^{2}}, \quad \varrho=-\frac{R}{s^{3}} .
$$

Evidently, the internal degree of freedom in the monkey-tail model has no direct physical meaning in relation to the original problem providing the target solution. Thus, at low frequencies the point mass may undergo extreme displacements, and in the static case the displacement is infinite. This lack of direct relationship with the original problem is a general property of the discrete-element models. They merely provide a mechanical system that leads to a similar frequency response.

\subsubsection{Second-order terms with one or two internal degrees of fredom}

The second-order term given by Eq. (89c) has four parameters: $\alpha_{0}, \alpha_{1}, \beta_{0}$ and $\beta_{1}$. An example of a second-order discrete-element model is shown in Fig. 11a. This particular model has two internal nodes. The equilibrium formulations for Nodes 0,1 and 2 (for harmonic loading) are as follows:

$$
\begin{array}{ll}
\text { Node 0 : } & \kappa_{1}\left(U_{0}(\omega)-U_{1}(\omega)\right)-\kappa_{1} U_{0}(\omega)=P_{0}(\omega) \\
\text { Node 1: } & \kappa_{1}\left(U_{1}(\omega)-U_{0}(\omega)\right)+\mathrm{i} \omega \gamma_{1} \frac{R_{0}}{c_{0}}\left(U_{1}(\omega)-U_{2}(\omega)\right)=0 \\
\text { Node 2: } & \kappa_{2} U_{2}(\omega)+\mathrm{i} \omega \gamma_{2} \frac{R_{0}}{c_{0}} U_{2}(\omega)+\mathrm{i} \omega \gamma_{1} \frac{R_{0}}{c_{0}}\left(U_{2}(\omega)-U_{1}(\omega)\right)=0 .
\end{array}
$$

After some rearrangement and elimination of the internal degrees of freedom, the force-displacement relation of the second-order model is given by

$$
P_{0}\left(a_{0}\right)=\frac{-\kappa_{1}^{2} \frac{\gamma_{1}+\gamma_{2}}{\gamma_{1} \gamma_{2}} \mathrm{i} a_{0}-\frac{\kappa_{1}^{2} \kappa_{2}}{\gamma_{1} \gamma_{2}}}{\left(\mathrm{i} a_{0}\right)^{2}+\left(\kappa_{1} \frac{\gamma_{1}+\gamma_{2}}{\gamma_{1} \gamma_{2}}+\frac{\kappa_{2}}{\gamma_{2}}\right) \mathrm{i} a_{0}+\frac{\kappa_{1} \kappa_{2}}{\gamma_{1} \gamma_{2}}} U_{0}\left(a_{0}\right) .
$$

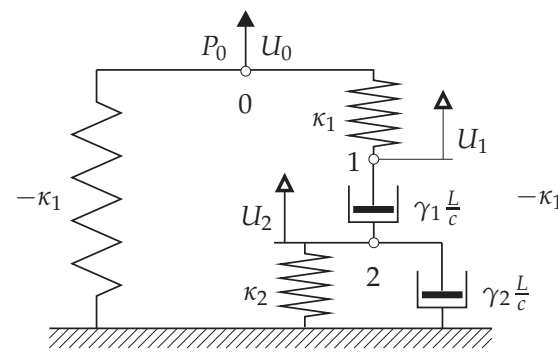

(a)

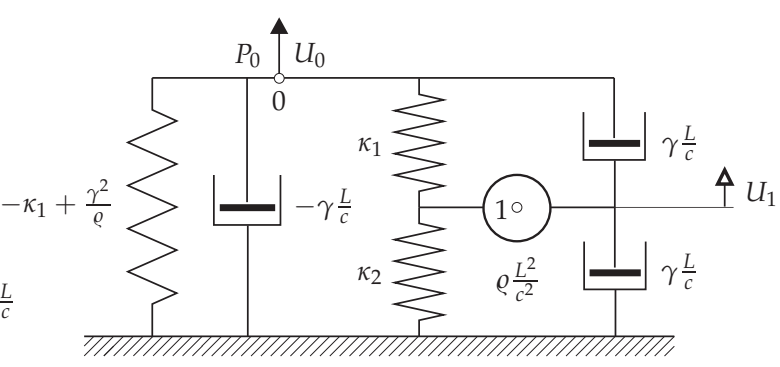

(b)

Fig. 11. The discrete-element model for the second-order term: (a) Spring-dashpot model with two internal degrees of freedom; (b) spring-dashpot-mass model with one internal degree of freedom. 
By a comparison of Eqs. (89c) and (97), the four coefficients in Eq. (97) are identified as

$$
\begin{gathered}
\kappa_{1}=-\frac{\beta_{0}}{\alpha_{0}}, \quad \gamma_{1}=-\frac{\alpha_{0} \beta_{1}-\alpha_{1} \beta_{0}}{\alpha_{0}^{2}}, \\
\kappa_{2}=\frac{\beta_{0}}{\alpha_{0}^{2}} \frac{\left(-\alpha_{0} \beta_{1}+\alpha_{1} \beta_{0}\right)^{2}}{\alpha_{0} \beta_{1}^{2}-\alpha_{1} \beta_{0} \beta_{1}+\beta_{0}^{2}}, \quad \gamma_{2}=\frac{\beta_{0}^{2}}{\alpha_{0}^{2}} \frac{-\alpha_{0} \beta_{1}+\alpha_{1} \beta_{0}}{\alpha_{0} \beta_{1}^{2}-\alpha_{1} \beta_{0} \beta_{1}+\beta_{0}^{2}} .
\end{gathered}
$$

Alternatively, introducing a second-order model with springs, dampers and a point mass, it is possible to construct a second-order model with only one internal degree of freedom. The model is sketched in Fig. 11b. The force-displacement relation of the alternative second-order model is given by

$$
P_{0}\left(a_{0}\right)=\frac{2\left(\frac{\kappa_{1} \gamma}{\varrho}+\frac{\gamma^{3}}{\varrho^{2}}\right) \mathrm{i} a_{0}-\frac{\kappa_{1}^{2}}{\varrho}+\frac{\left(\kappa_{1}+\kappa_{2}\right) \gamma^{2}}{\varrho^{2}}}{\left(\mathrm{i} a_{0}\right)^{2}+2 \frac{\gamma}{\varrho} \mathrm{i} a_{0}+\frac{\kappa_{1}+\kappa_{2}}{\varrho}} U_{0}\left(a_{0}\right) .
$$

By equating the coefficients in Eq. (99) to the terms of the second-order model in Eq. (89c), the four parameters $\kappa_{1}, \kappa_{2}, \gamma$ and $\varrho$ can be determined. In order to calculate $\varrho$, a quadratic equation has to be solved. The quadratic equation for $\varrho$ is

$$
a \varrho^{2}+b \varrho+c=0 \quad \text { where } \quad a=\alpha_{1}^{4}-4 \alpha_{0} \alpha_{1}^{2}, \quad b=-8 \alpha_{1} \beta_{1}+16 \beta_{0}, \quad c=16 \frac{\beta_{1}^{2}}{\alpha_{1}^{2}} .
$$

Equation (100) results in two solutions for $\varrho$. To ensure real values of $\varrho, b^{2}-4 a c \geq 0$ or $\alpha_{0} \beta_{1}^{2}-\alpha_{1} \beta_{0} \beta_{1}+\beta_{0}^{2} \geq 0$. When $\varrho$ has been determined, the three remaining coefficients can be calculated by

$$
\kappa_{1}=\frac{\varrho \alpha_{1}^{2}}{4}-\frac{\beta_{1}}{\alpha_{1}}, \quad \kappa_{2}=\varrho \alpha_{0}-\kappa_{1}, \quad \gamma=\frac{\varrho \alpha_{1}}{2} .
$$

\subsection{Fitting of a rational filter}

In order to get a stable solution in the time domain, the poles of $\widehat{S}_{r}\left(\mathrm{i} a_{0}\right)$ should all reside in the second and third quadrant of the complex plane, i.e. the real parts of the poles must all be negative. Due to the fact that computers only have a finite precision, this requirement may have to be adjusted to $s_{m}<-\varepsilon, m=1,2, \ldots, M$, where $\varepsilon$ is a small number, e.g. 0.01 .

The rational approximation may now be obtained by curve-fitting of the rational filter $\widehat{S}_{r}\left(\mathrm{i} a_{0}\right)$ to the regular part of the dynamic stiffness, $S_{r}\left(a_{0}\right)$, by a least-squares technique. In this process, it should be observed that:

1. The response should be accurately described by the lumped-parameter model in the frequency range that is important for the physical problem being investigated. For soil-structure interaction of wind turbines, this is typically the low-frequency range.

2. The "exact" values of $S_{r}\left(a_{0}\right)$ are only measured—or computed-over a finite range of frequencies, typically for $a_{0} \in\left[0 ; a_{0 \max }\right]$ with $a_{0 \max }=2 \sim 10$. Further, the values of $S_{r}\left(a_{0}\right)$ are typically only known at a number of discrete frequencies.

3. Outside the frequency range, in which $S_{r}\left(a_{0}\right)$ has been provided, the singular part of the dynamic stiffness, $S_{S}\left(a_{0}\right)$, should govern the response. Hence, no additional tips and dips should appear in the frequency response provided by the rational filter beyond the dimensionless frequency $a_{0 \max }$. 
Firstly, this implies that the order of the filter, $M$, should not be too high. Experience shows that orders about $M=2 \sim 8$ are adequate for most physical problems. Higher-order filters than this are not easily fitted, and lower-order filters provide a poor match to the "exact" results. Secondly, in order to ensure a good fit of $\widehat{S}_{r}\left(\mathrm{i} a_{0}\right)$ to $S_{r}\left(a_{0}\right)$ in the low-frequency range, it is recommended to employ a higher weight on the squared errors in the low-frequency range, e.g. for $a_{0}<0.2 \sim 2$, compared with the weights in the medium-to-high-frequency range. Obviously, the definition of low, medium and high frequencies is strongly dependent on the problem in question. For example, frequencies that are considered high for an offshore wind turbine, may be considered low for a diesel power generator.

For soil-structure interaction of foundations, Wolf (1994) suggested to employ a weight of $w\left(a_{0}\right)=10^{3} \sim 10^{5}$ at low frequencies and unit weight at higher frequencies. This should lead to a good approximation in most cases. However, numerical experiment indicates that the fitting goodness of the rational filter is highly sensitive to the choice of the weight function $w\left(a_{0}\right)$, and the guidelines provided by Wolf (1994) are not useful in all situations. Hence, as an alternative, the following fairly general weight function is proposed:

$$
w\left(a_{0}\right)=\frac{1}{\left(1+\left(\varsigma_{1} a_{0}\right)^{\zeta_{2}}\right)^{\zeta_{3}}} .
$$

The coefficients $\varsigma_{1}, \varsigma_{2}$ and $\varsigma_{3}$ are heuristic parameters. Experience shows that values of about $\varsigma_{1}=\varsigma_{2}=\varsigma_{3}=2$ provide an adequate solution for most foundations in the low-frequency range $a_{0} \in[0 ; 2]$. This recommendation is justified by the examples given in the next section. For analyses involving high-frequency excitation, lower values of $\varsigma_{1}, \varsigma_{2}$ and $\varsigma_{3}$ may have to be employed.

Hence, the optimisation problem defined in Table 1. However, the requirement of all poles lying in the second and third quadrant of the complex plane is not easily fulfilled when an optimisation is carried out by least-squares (or similar) curve fitting of $\widehat{S}_{r}\left(\mathrm{i} a_{0}\right)$ to $S_{r}\left(a_{0}\right)$ as suggested in Table 1. Specifically, the choice of the polynomial coefficients $q_{j}, j=1,2, \ldots, m$, as the optimisation variables is unsuitable, since the constraint that all poles of $\widehat{S}_{r}\left(\mathrm{i} a_{0}\right)$ must have negative real parts is not easily incorporated in the optimisation problem. Therefore, instead of the interpretation

$$
Q\left(\mathrm{i} a_{0}\right)=1+q_{1}\left(\mathrm{i} a_{0}\right)+q_{2}\left(\mathrm{i} a_{0}\right)^{2}+\ldots+q_{M}\left(\mathrm{i} a_{0}\right)^{M},
$$

an alternative approach is considered, in which the denominator is expressed as

$$
Q\left(\mathrm{i} a_{0}\right)=\left(\mathrm{i} a_{0}-s_{1}\right)\left(\mathrm{i} a_{0}-s_{2}\right) \cdots\left(\mathrm{i} a_{0}-s_{M}\right)=\prod_{m=1}^{M}\left(\mathrm{i} a_{0}-s_{m}\right) .
$$

In this representation, $s_{m}, m=1,2, \ldots, M$, are the roots of $Q\left(\mathrm{i} a_{0}\right)$. In particular, if there are $N$ complex conjugate pairs, the denominator polynomial may advantageously be expressed as

$$
Q\left(\mathrm{i} a_{0}\right)=\prod_{n=1}^{N}\left(\mathrm{i} a_{0}-s_{n}\right)\left(\mathrm{i} a_{0}-s_{n}^{*}\right) \cdot \prod_{n=N+1}^{M-N}\left(\mathrm{i} a_{0}-s_{n}\right) .
$$

where an asterisk $(*)$ denotes the complex conjugate. Thus, instead of the polynomial coefficients, the roots $s_{n}$ are identified as the optimisation variables. 
A rational filter for the regular part of the dynamic stiffness is defined in the form:

$$
S_{r}\left(a_{0}\right) \approx \widehat{S}_{r}\left(\mathrm{i} a_{0}\right)=\frac{P\left(\mathrm{i} a_{0}\right)}{Q\left(\mathrm{i} a_{0}\right)}=\frac{1-k^{\infty}+p_{1}\left(\mathrm{i} a_{0}\right)+p_{2}\left(\mathrm{i} a_{0}\right)^{2}+\ldots+p_{M-1}\left(\mathrm{i} a_{0}\right)^{M-1}}{1+q_{1}\left(\mathrm{i} a_{0}\right)+q_{2}\left(\mathrm{i} a_{0}\right)^{2}+\ldots+q_{M}\left(\mathrm{i} a_{0}\right)^{M}} .
$$

Find the optimal polynomial coefficients $p_{n}$ and $q_{m}$ which minimize the object function $F\left(p_{n}, q_{m}\right)$ in a weighted-least-squares sense subject to the constraints $G_{1}\left(p_{n}, q_{m}\right), G_{2}\left(p_{n}, q_{m}\right), \ldots, G_{M}\left(p_{n}, q_{m}\right)$.

Input:

$M: \quad$ order of the filter

$p_{n}^{0}, \quad n=1,2, \ldots, M-1$,

$q_{m}^{0}, \quad m=1,2, \ldots, M$,

$a_{0 j}, \quad j=1,2, \ldots, J$,

$S_{r}\left(a_{0 j}\right), \quad j=1,2, \ldots, J$,

$w\left(a_{0 j}\right), \quad j=1,2, \ldots, J$.

Variables:

$$
\begin{array}{ll}
p_{n}, & n=1,2, \ldots, M-1, \\
q_{m}, & m=1,2, \ldots, M .
\end{array}
$$

Object function:

$$
F\left(p_{n}, q_{m}\right)=\sum_{j=1}^{J} w\left(a_{0 j}\right)\left(\widehat{S}_{r}\left(\mathrm{i} a_{0 j}\right)-S_{r}\left(a_{0 j}\right)\right)^{2} .
$$

Constraints:

$$
\begin{gathered}
G_{1}\left(p_{n}, q_{m}\right)=\Re\left(s_{1}\right)<-\varepsilon, \\
G_{2}\left(p_{n}, q_{m}\right)=\Re\left(s_{2}\right)<-\varepsilon, \\
\vdots \\
G_{M}\left(p_{n}, q_{m}\right)=\Re\left(s_{M}\right)<-\varepsilon .
\end{gathered}
$$

Output:

$$
\begin{array}{ll}
p_{n}, & n=1,2, \ldots, M-1, \\
q_{m}, & m=1,2, \ldots, M .
\end{array}
$$

Here, $p_{n}^{0}$ and $q_{m}^{0}$ are the initial values of the polynomial coefficients $p_{n}$ and $q_{m}$, whereas $S_{r}\left(a_{0 j}\right)$ are the "exact" value of the dynamic stiffness evaluated at the $J$ discrete dimensionless frequencies $a_{0 j}$. These are either measured or calculated by rigourous numerical or analytical methods. Further, $\widehat{S}_{r}\left(\mathrm{i} a_{0 j}\right)$ are the values of the rational filter at the same discrete frequencies, and $w\left(a_{0}\right)$ is a weight function, e.g. as defined by Eq. (102) with $\varsigma_{1}=\varsigma_{2}=\varsigma_{3}=2$. Finally, $s_{m}$ are the poles of the rational filter $\widehat{S}_{r}\left(\mathrm{i} a_{0}\right)$, i.e. the roots of the denominator polynomial $Q\left(\mathrm{i} a_{0}\right)$, and $\varepsilon$ is a small number, e.g. $\varepsilon=0.01$.

Table 1. Fitting of rational filter by optimisation of polynomial coefficients.

Accordingly, in addition to the coefficients of the numerator polynomial $P\left(\mathrm{i} a_{0}\right)$, the variables in the optimisation problem are the real and imaginary parts $s_{n}^{\Re}=\Re\left(s_{n}\right)$ and $s_{n}^{\Im}=\Im\left(s_{n}\right)$ of the complex roots $s_{n}, n=1,2, \ldots, N$, and the real roots $s_{n}, n=N+1, N+2, \ldots, M-N$.

The great advantage of the representation (105) is that the constraints on the poles are defined directly on each individual variable, whereas the constraints in the formulation with $Q\left(\mathrm{i} a_{0}\right)$ defined by Eq. (103), the constraints are given on functionals of the variables. Hence, the solution is much more efficient and straightforward. However, Eq. (105) has two disadvantages when compared with Eq. (103):

- The number of complex conjugate pairs has to be estimated. However, experience shows that as many as possible of the roots should appear as complex conjugates-e.g. if $M$ is even, $N=M / 2$ should be utilized. This provides a good fit in most situations and may, at the same time, generate the lumped-parameter model with fewest possible internal degrees of freedom. 
A rational filter for the regular part of the dynamic stiffness is defined in the form:

$$
S_{r}\left(a_{0}\right) \approx \widehat{S}_{r}\left(\mathrm{i} a_{0}\right)=\frac{P\left(\mathrm{i} a_{0}\right)}{Q\left(\mathrm{i} a_{0}\right)}=\frac{1-k^{\infty}+p_{1}\left(\mathrm{i} a_{0}\right)+p_{2}\left(\mathrm{i} a_{0}\right)^{2}+\ldots+p_{M-1}\left(\mathrm{i} a_{0}\right)^{M-1}}{\prod_{m=1}^{N}\left(\mathrm{i} a_{0}-s_{m}\right)\left(\mathrm{i} a_{0}-s_{m}^{*}\right) \cdot \prod_{m=N+1}^{M-N}\left(\mathrm{i} a_{0}-s_{m}\right)} .
$$

Find the optimal polynomial coefficients $p_{n}$ and the poles $s_{m}$ which minimise the object function $F\left(p_{n}, s_{m}\right)$ subject to the constraints $G_{0}\left(p_{n}, s_{m}\right), G_{1}\left(p_{n}, s_{m}\right), \ldots, G_{N}\left(p_{n}, s_{m}\right)$.

Input: $\quad M:$ order of the filter

$N$ : number of complex conjugate pairs, $2 N \leq M$

$p_{n}^{0}, \quad n=1,2, \ldots, M-1$,

$s_{m}^{\Re 0}, \quad m=1,2, \ldots, N$,

$s_{m}^{\Im 0}, \quad m=1,2, \ldots, N$,

$s_{m}^{0}, \quad m=1,2, \ldots, M-N$,

$a_{0 j}, \quad j=1,2, \ldots, J$,

$S_{r}\left(a_{0 j}\right), \quad j=1,2, \ldots, J$,

$w\left(a_{0 j}\right), \quad j=1,2, \ldots, J$.

Variables:

$$
\begin{array}{lll}
p_{n,} & n=1,2, \ldots, M-1, & \\
s_{m,}^{\Re,} & m=1,2, \ldots, N, & s_{m}^{\Re}<-\varepsilon, \\
s_{m,}^{\Im} & m=1,2, \ldots, N, & s_{m}^{\Im}>+\varepsilon, \\
s_{m}, & m=N+1,2, \ldots, M-N, & s_{m}<-\varepsilon .
\end{array}
$$

Object function: $\quad F\left(p_{n}, s_{m}\right)=\sum_{j=1}^{J} w\left(a_{0 j}\right)\left(\widehat{S}_{r}\left(\mathrm{i} a_{0 j}\right)-S_{r}\left(a_{0 j}\right)\right)^{2}$.

Constraints: $\quad G_{0}\left(p_{n}, s_{m}\right)=1-\prod_{1}^{M}\left(-s_{m}\right)=0$,

$$
G_{k}\left(p_{n}, s_{m}\right)=\zeta s_{k}^{\Re}+s_{k}^{\Im}<0, \quad k=1,2, \ldots, N \text {. }
$$

Output:

$$
\begin{array}{ll}
p_{n,}, & n=1,2, \ldots, M-1, \\
s_{m,}^{\Re,} & m=1,2, \ldots, N, \\
s_{m}^{\Im} & m=1,2, \ldots, N, \\
s_{m}, & m=N+1,2, \ldots, M-N .
\end{array}
$$

Here, superscript 0 indicates initial values of the respective variables, and $\widehat{S}_{r}\left(\mathrm{i} a_{0 j}\right)$ are the values of the rational filter at the same discrete frequencies. Further, $\zeta \approx 10 \sim 100$ and $\varepsilon \approx 0.01$ are two real parameters. Note that the initial values of the poles must conform with the constraint $G_{0}\left(p_{n}, s_{m}\right)$. For additional information, see Table 1.

Table 2. Fitting of rational filter by optimisation of the poles.

- In the representation provided by Eq. (103), the correct asymptotic behaviour is automatically ensured in the limit $\mathrm{i} a_{0} \rightarrow 0$, i.e. the static case, since $q_{0}=1$. Unfortunately, in the representation given by Eq. (105) an additional equality constraint has to be implemented to ensure this behaviour. However, this condition is much easier implemented than the constraints which are necessary in the case of Eq. (103) in order to prevent the real parts of the roots from being positive.

Eventually, instead of the problem defined in Table 1, it may be more efficient to solve the optimisation problem given in Table 2. It is noted that additional constraints are suggested, which prevent the imaginary parts of the complex poles to become much (e.g. 10 times) bigger than the real parts. This is due to the following reason: If the real part of the complex pole $s_{m}$ vanishes, i.e. $s_{m}^{\Re}=0$, this results in a second order pole, $\left\{s_{m}^{\Im}\right\}^{2}$, which is real and positive. 
Evidently, this will lead to instability in the time domain. Since the computer precision is limited, a real part of a certain size compared to the imaginary part of the pole is necessary to ensure a stable solution.

Finally, as an alternative to the optimisation problems defined in Table 1 and Table 2, the function $S\left(\mathrm{i} a_{0}\right)$ may be expressed by Eq. (86), i.e. in partial-fraction form. In this case, the variables in the optimization problem are the poles and residues of $S\left(\mathrm{i} a_{0}\right)$. In the case of the second-order terms, these quantities are replaced with $\alpha_{0}, \alpha_{1}, \beta_{0}$ and $\beta_{1}$. At a first glance, this choice of optimisation variable seems more natural than $p_{n}$ and $s_{m}$, as suggested in Table 2. However, from a computational point of view, the mathematical operations involved in the polynomial-fraction form are more efficient than those of the polynomial-fraction form. Hence, the scheme provided in Table 2 is recommended.

\section{Time-domain analysis of soil-structure interaction}

In this section, two examples are given in which consistent lumped-parameter models are applied to the analysis of foundations and soil-structure interaction. The first example concerns a rigid hexagonal footing on a homogeneous or layered ground and was first presented by Andersen (2010). The frequency-domain solution obtained by the domain-transformation method presented in Sections 2-3 is fitted by LPMs of different orders. Subsequently, the response of the original model and the LPMs are compared in frequency and time domain.

In the second example, originally proposed by Andersen et al. (2009), LPMs are fitted to the frequency-domain results of a coupled boundary-element/finite-element model of a flexible embedded foundation. As part of the examples, the complex stiffness of the foundation in the high-frequency limit is discussed, i.e. the coefficients $k^{\infty}$ and $c^{\infty}$ in Eq. (82) are determined for each component of translation and rotation of the foundation. Whereas no coupling exists between horizontal sliding and rocking of surface footings in the high-frequency limit, a significant coupling is present in the case of embedded foundations-even at high frequencies.

\subsection{Example: A footing on a homogeneous or layered ground}

The foundation is modelled as a regular hexagonal rigid footing with the side length $r_{0}$, height $h_{0}$ and mass density $\rho_{0}$. This geometry is typical for offshore wind turbine foundations.

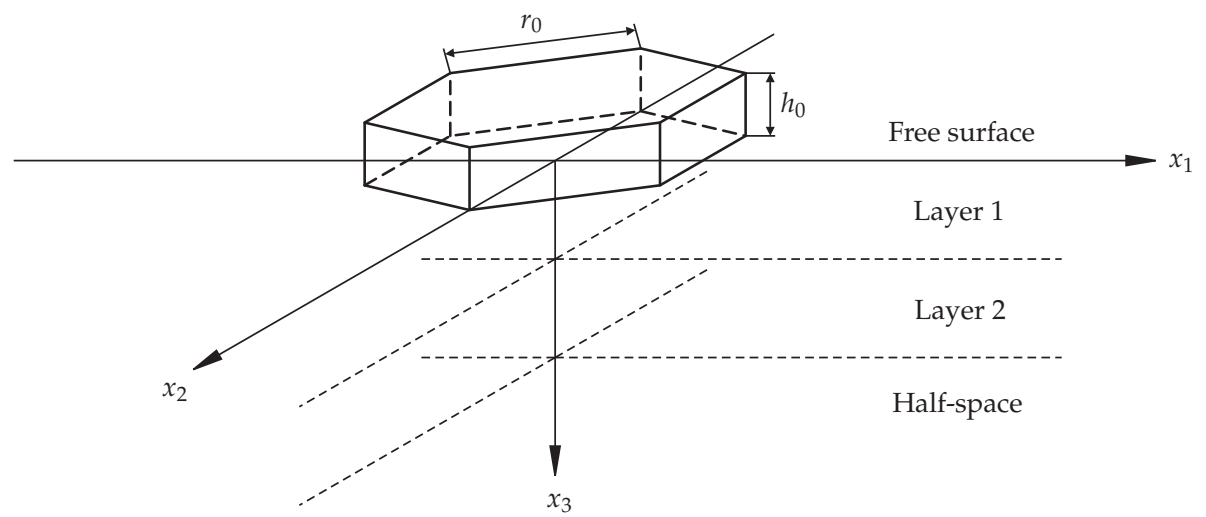

Fig. 12. Hexagonal footing on a stratum with three layers over a half-space. 
As illustrated in Fig. 12, the centre of the soil-foundation interface coincides with the origin of the Cartesian coordinate system. The mass of the foundation and the corresponding mass moments of inertia with respect to the three coordinate axes then become:

$$
M_{0}=\rho_{0} h_{0} A_{0}, \quad \mathcal{J}_{1}=\mathcal{J}_{2}=\rho_{0} h_{0} \mathcal{I}_{0}+\frac{1}{3} \rho_{0} h_{0}^{3} A_{0}, \quad \mathcal{J}_{3}=2 \rho_{0} h_{0} \mathcal{I}_{0}
$$

where $A_{0}$ is the area of the horizontal cross-section and $\mathcal{I}_{0}$ is the corresponding geometrical moment of inertia,

$$
A_{0}=\frac{3 \sqrt{3}}{2} r_{0}^{2}, \quad \mathcal{I}_{0}=\frac{5 \sqrt{3}}{16} r_{0}^{4} .
$$

It is noted that $\mathcal{I}_{0}$ is invariant to rotation of the foundation around the $x_{3}$-axis. This property also applies to circular or quadratic foundations as discussed in Section 3.

\subsubsection{A footing on a homogeneous ground}

Firstly, we consider a hexagonal footing on a homogeneous visco-elastic half-space. The footing has the side length $r_{0}=10 \mathrm{~m}$, the height $h_{0}=10 \mathrm{~m}$ and the mass density $\rho_{0}=2000 \mathrm{~kg} / \mathrm{m}^{3}$, and the mass and mass moments of inertia are computed by Eq. (106). The properties of the soil are $\rho^{1}=2000 \mathrm{~kg} / \mathrm{m}^{3}, E^{1}=10^{4} \mathrm{kPa}, v^{1}=0.25$ and $\eta^{1}=0.03$. However, in the static limit, i.e. for $\omega \rightarrow 0$, the hysteretic damping model leads to a complex impedance in the frequency domain. By contrast, the lumped-parameter model provides a real impedance, since it is based on viscous dashpots. This discrepancy leads to numerical difficulties in the fitting procedure and to overcome this, the hysteretic damping model for the soil is replaced by a linear viscous model at low frequencies, in this case below $1 \mathrm{~Hz}$.

In principle, the time-domain solution for the displacements and rotations of the rigid footing is found by inverse Fourier transformation, i.e.

$$
v_{i}(t)=\frac{1}{2 \pi} \int_{-\infty}^{\infty} V_{i}(\omega) \mathrm{e}^{\mathrm{i} \omega t} \mathrm{~d} \omega, \quad \theta_{i}(t)=\frac{1}{2 \pi} \int_{-\infty}^{\infty} \Theta_{i}(\omega) \mathrm{e}^{\mathrm{i} \omega t} \mathrm{~d} \omega, \quad i=1,2,3 .
$$

The displacements, rotations, forces and moments in the time domain are visualised in Fig. 13. In the numerical computations, the frequency response spectrum is discretized and accordingly, the time-domain solution is found by a Fourier series.

According to Eqs. (72) and (74), the vertical motion $V_{3}(\omega)$ as well as the torsional motion $\Theta_{3}(\omega)$ (see Fig. 6) are decoupled from the remaining degrees of freedom of the hexagonal footing. Thus, $V_{3}(\omega)$ and $\Theta_{3}(\omega)$ may be fitted by independent lumped-parameter models.

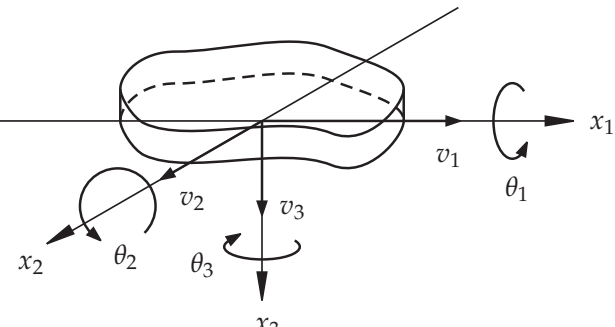

(a)

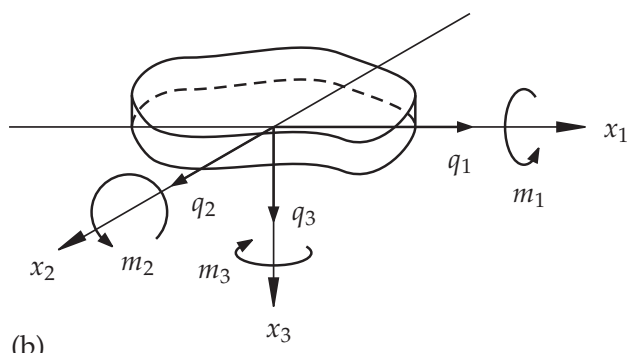

(b)

Fig. 13. Degrees of freedom for a rigid surface footing in the time domain: (a) displacements and rotations, and (b) forces and moments. 
In the following, the quality of lumped-parameter models based on rational filters of different orders are tested for vertical and torsional excitation.

For the footing on the homogeneous half-space, rational filters of the order 2-6 are tested. Firstly, the impedance components are determined in the frequency-domain by the method presented in Section 2. The lumped-parameter models are then fitted by application of the procedure described in Section 4 and summarised in Table 2. The two components of the normalised impedance, $S_{33}$ and $S_{66}$, are shown in Figs. 14 and 16 as functions of the physical frequency, $f$. It is noted that all the LPMs are based on second-order discrete-element models including a point mass, see Fig. 11b. Hence, the LPM for each individual component of the impedance matrix, $\mathbf{Z}(\omega)$, has 1, 2 or 3 internal degrees of freedom.

With reference to Fig. 14, a poor fit of the vertical impedance is obtained with $M=2$ regarding the absolute value of $S_{33}$ as well as the phase angle. A lumped-parameter model with $M=4$ provides a much better fit in the low-frequency range. However, a sixth-order lumped-parameter model is required to obtain an accurate solution in the medium-frequency range, i.e. for frequencies between approximately 1.5 and $4 \mathrm{~Hz}$. As expected, further analyses show that a slightly better match in the medium-frequency range is obtained with the weight-function coefficients $\varsigma_{1}=2$ and $\varsigma_{2}=\varsigma_{3}=1$. However, this comes at the cost of a poorer match in the low-frequency range. Finally, it has been found that no improvement is achieved if first-order terms, e.g. the "monkey tail" illustrated in Fig. 10b, are allowed in the rational-filter approximation.

Figure 16 shows the rational-filter approximations of $S_{66}$, i.e. the non-dimensional torsional impedance. Compared with the results for the vertical impedance, the overall quality of the fit is relatively poor. In particular the LPM with $M=2$ provides a phase angle which is negative in the low frequency range. Actually, this means that the geometrical damping provided by the second-order LPM becomes negative for low-frequency excitation. Furthermore, the stiffness is generally under-predicted and as a consequence of this an LPM with $M=2$ cannot be used for torsional vibrations of the surface footing.

A significant improvement is achieved with $M=4$, but even with $M=6$ some discrepancies are observed between the results provide by the LPM and the rigorous model. Unfortunately, additional studies indicate that an LPM with $M=8$ does not increase the accuracy beyond that of the sixth-order model.

Next, the dynamic soil-foundation interaction is studied in the time domain. In order to examine the transient response, a pulse load is applied in the form

$$
p(t)= \begin{cases}\sin \left(2 \pi f_{c} t\right) \sin \left(0.5 \pi f_{c} t\right) & \text { for } \quad 0<t<2 / f_{c} \\ 0 & \text { otherwise. }\end{cases}
$$

In this analysis, $f_{c}=2 \mathrm{~Hz}$ is utilised, and the responses obtained with the lumped-parameter models of different orders are computed by application of the Newmark $\beta$-scheme proposed by Newmark Newmark (1959). Figure 15 shows the results of the analysis with $q_{3}(t)=p(t)$, whereas the results for $m_{3}(t)=p(t)$ are given in Fig. 17 .

In the case of vertical excitation, Fig. 15 shows that even the LPM with $M=2$ provides an acceptable match to the "exact" results achieved by inverse Fourier transformation of the frequency-domain solution. In particular, the maximum response occurring during the excitation is well described. However, an improvement in the description of the damping is obtained with $M=4$. For torsional motion, the second-order LPM is invalid since it provides negative damping. Hence, the models with $M=4$ and $M=6$ are compared in Fig. 17. It is clearly demonstrated that the fourth-order LPM provides a poor representation of the 
torsional impedance, whereas an accurate prediction of the response is achieved with the sixth-order model.

Subsequently, lumped-parameter models are fitted for the horizontal sliding and rocking motion of the surface footing, i.e. $V_{2}(\omega)$ and $\Theta_{1}(\omega)$ (see Fig. 6). As indicated by Eqs. (72) and (74), these degrees of freedom are coupled via the impedance component $Z_{24}$. Hence, two analyses are carried out. Firstly, the quality of lumped-parameter models based on rational filters of different orders are tested for horizontal and moment excitation. Secondly, the significance of coupling is investigated by a comparison of models with and without the coupling terms.

Similarly to the case for vertical and torsional motion, rational filters of the order 2-6 are tested. The three components of the normalised impedance, $S_{22}, S_{24}=S_{42}$ and $S_{44}$, are shown in Figs. 18, 20 and 22 as functions of the physical frequency, $f$. Again, the lumped-parameter models are based on discrete-element model shown in Fig. 11b, which reduces the number of internal degrees of freedom to a minimum. Clearly, the lumped-parameter models with $M=2$ provide a poor fit for all the components $S_{22}, S_{24}$ and $S_{44}$. However, Figs. 18 and 22 show that an accurate solution is obtained for $S_{22}$ and $S_{44}$ when a 4 th model is applied, and the inclusion of an additional internal degree of freedom, i.e. raising the order from $M=4$ to $M=6$, does not increase the accuracy significantly. However, for $S_{24}$ an LPM with $M=6$ is much more accurate than an LPM with $M=4$ for frequencies $f>3 \mathrm{~Hz}$, see Fig. 20.

Subsequently, the transient response to the previously defined pulse load with centre frequency $f_{c}=2 \mathrm{~Hz}$ is studied. Figure 19 shows the results of the analysis with $q_{2}(t)=p(t)$, and the results for $m_{1}(t)=p(t)$ are given in Fig. 21. Further, the results from an alternative analysis with no coupling of sliding and rocking are presented in Fig. 23. In Fig. 19 it is observed that the LPM with $M=2$ provides a poor match to the results of the rigorous model. The maximum response occurring during the excitation is well described by the low-order LPM. However the damping is significantly underestimated by the LPM. Since the loss factor is small, this leads to the conclusion that the geometrical damping is not predicted with adequate accuracy. On the other hand, for $M=4$ a good approximation is obtained with regard to both the maximum response and the geometrical damping. As suggested by Fig. 18, almost no further improvement is gained with $M=6$. For the rocking produced by a moment applied to the rigid footing, the lumped-parameter model with $M=2$ is useless. Here, the geometrical damping is apparently negative. However, $M=4$ provides an accurate solution (see Fig. 21) and little improvement is achieved by raising the order to $M=6$ (this result is not included in the figure).

Alternatively, Fig. 23 shows the result of the time-domain solution for a lumped-parameter model in which the coupling between sliding and rocking is disregarded. This model is interesting because the two coupling components $S_{24}$ and $S_{42}$ must be described by separate lumped-parameter models. Thus, the model with $M=4$ in Fig. 23 has four less internal degrees of freedom than the corresponding model with $M=4$ in Fig. 21. However, the two results are almost identical, i.e. the coupling is not pronounced for the footing on the homogeneous half-space. Hence, the sliding-rocking coupling may be disregarded without significant loss of accuracy. Increasing the order of the LPMs for $S_{22}$ and $S_{44}$ from 4 to 8 results in a model with the same number of internal degrees of freedom as the fourth-order model with coupling; but as indicated by Fig. 23, this does not improve the overall accuracy. Finally, Fig. 20 suggests that the coupling is more pronounced when a load with, for example, $f_{c}=1.5$ or $3.5 \mathrm{~Hz}$ is applied. However, further analyses, whose results are not presented in this paper, indicate that this is not the case. 

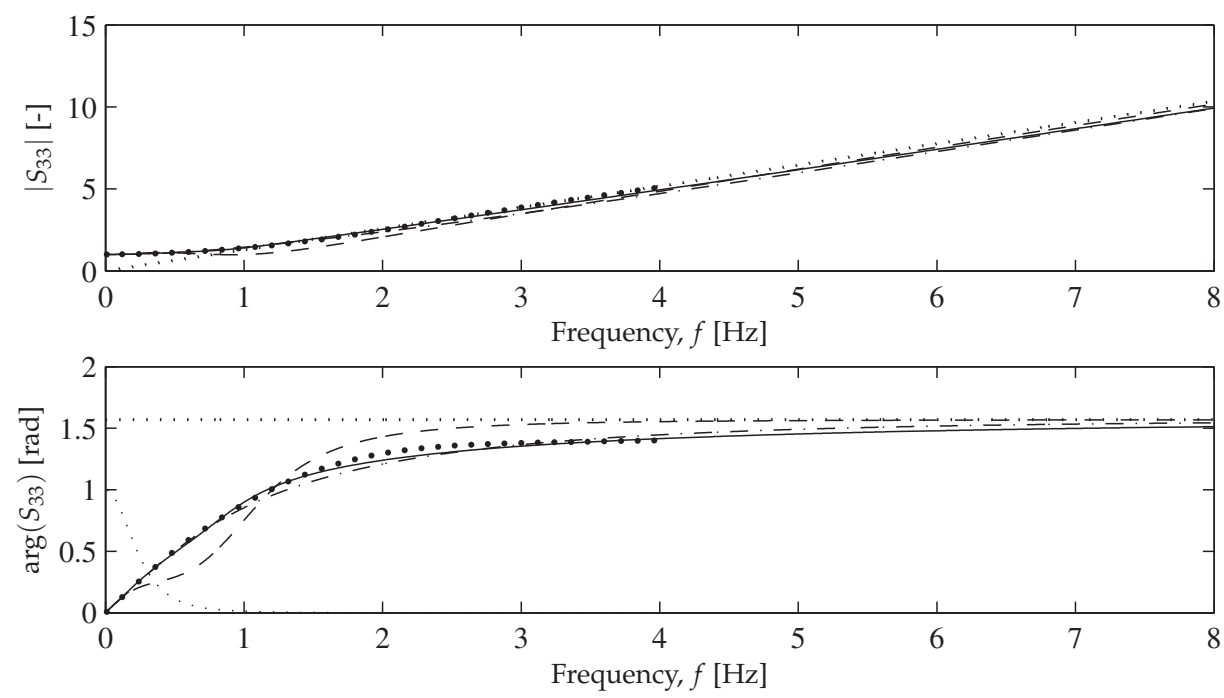

Fig. 14. Dynamic stiffness coefficient, $S_{33}$, obtained by the domain-transformation model (the large dots) and lumped-parameter models with $M=2(--), M=4(-\cdot-\cdot)$, and $M=6$ $(-)$. The thin dotted line (....$)$ ) indicates the weight function $w$ (not in radians), and the thick dotted line $(\cdots \cdots)$ indicates the high-frequency solution, i.e. the singular part of $S_{33}$.
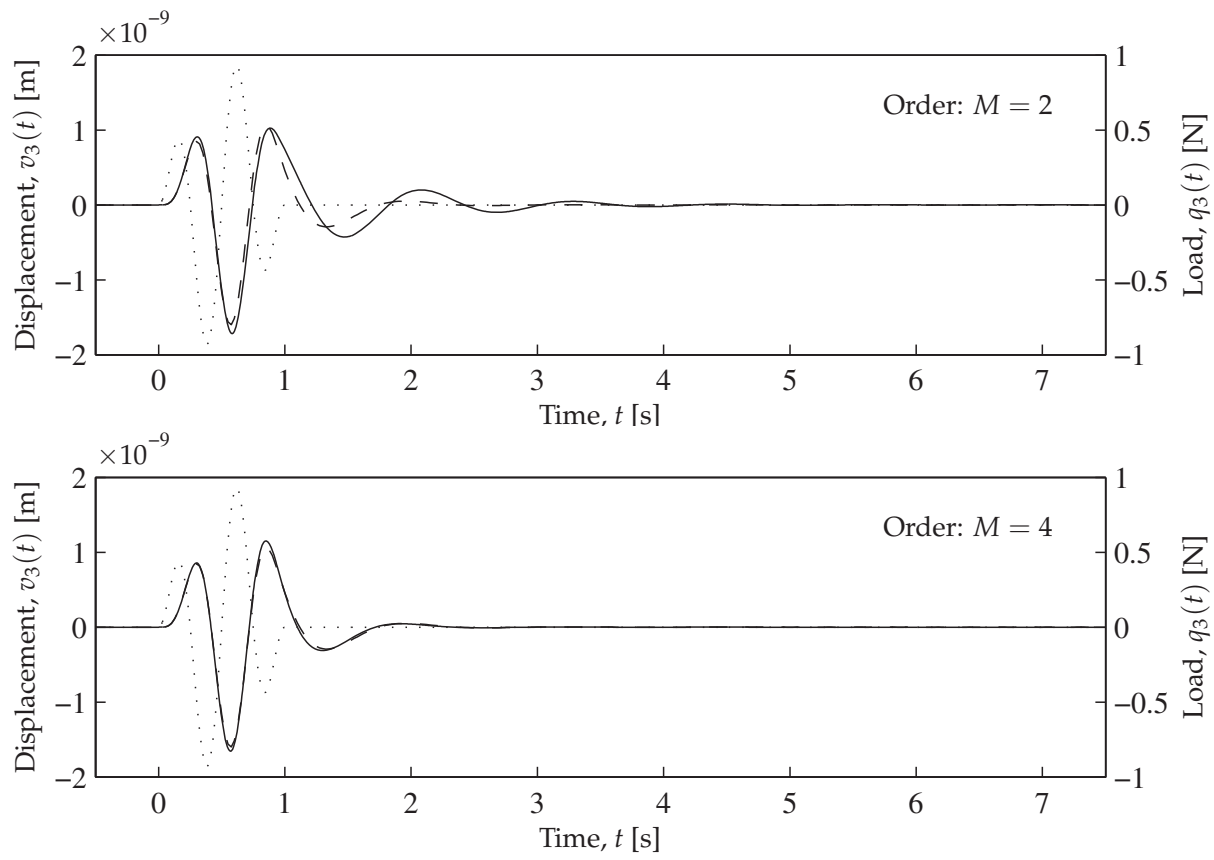

Fig. 15. Response $v_{3}(t)$ obtained by inverse Fourier transformation $(--)$ and lumped-parameter model $(-)$. The dots $(\cdots \cdots)$ indicate the load time history. 

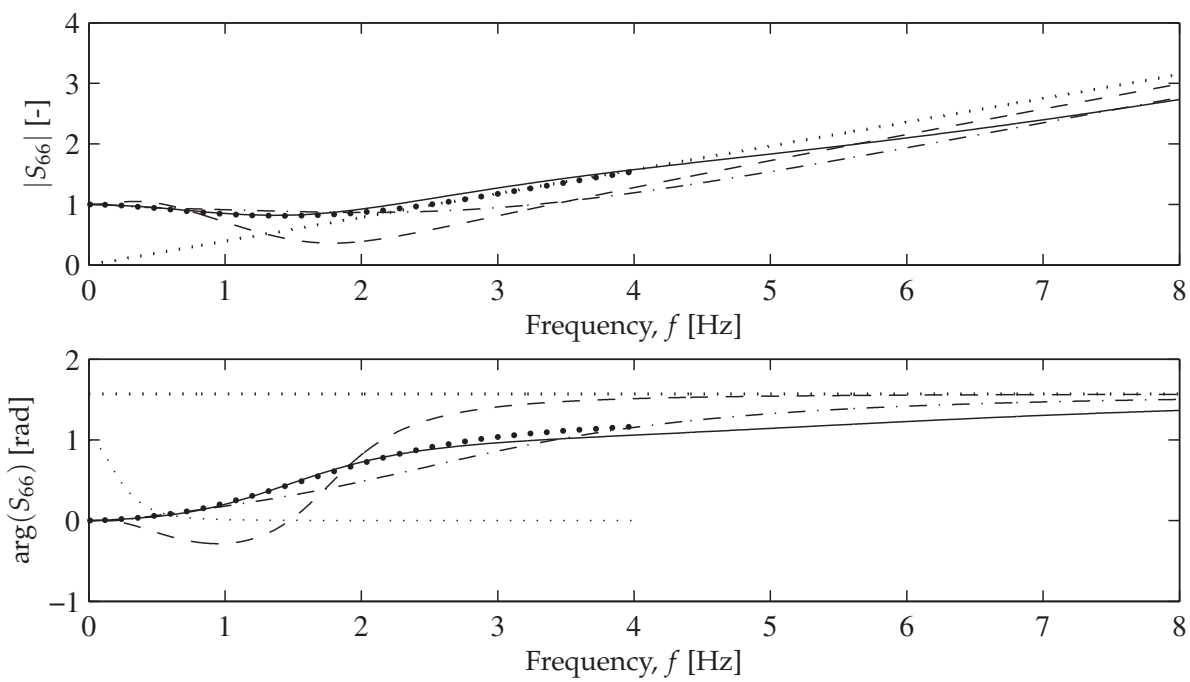

Fig. 16. Dynamic stiffness coefficient, $S_{66}$, obtained by the domain-transformation model (the large dots) and lumped-parameter models with $M=2(--), M=4(-\cdot-\cdot)$, and $M=6$ $(-)$. The thin dotted line ( ......) indicates the weight function $w$ (not in radians), and the thick dotted line $(\cdots \cdots)$ indicates the high-frequency solution, i.e. the singular part of $S_{66}$.
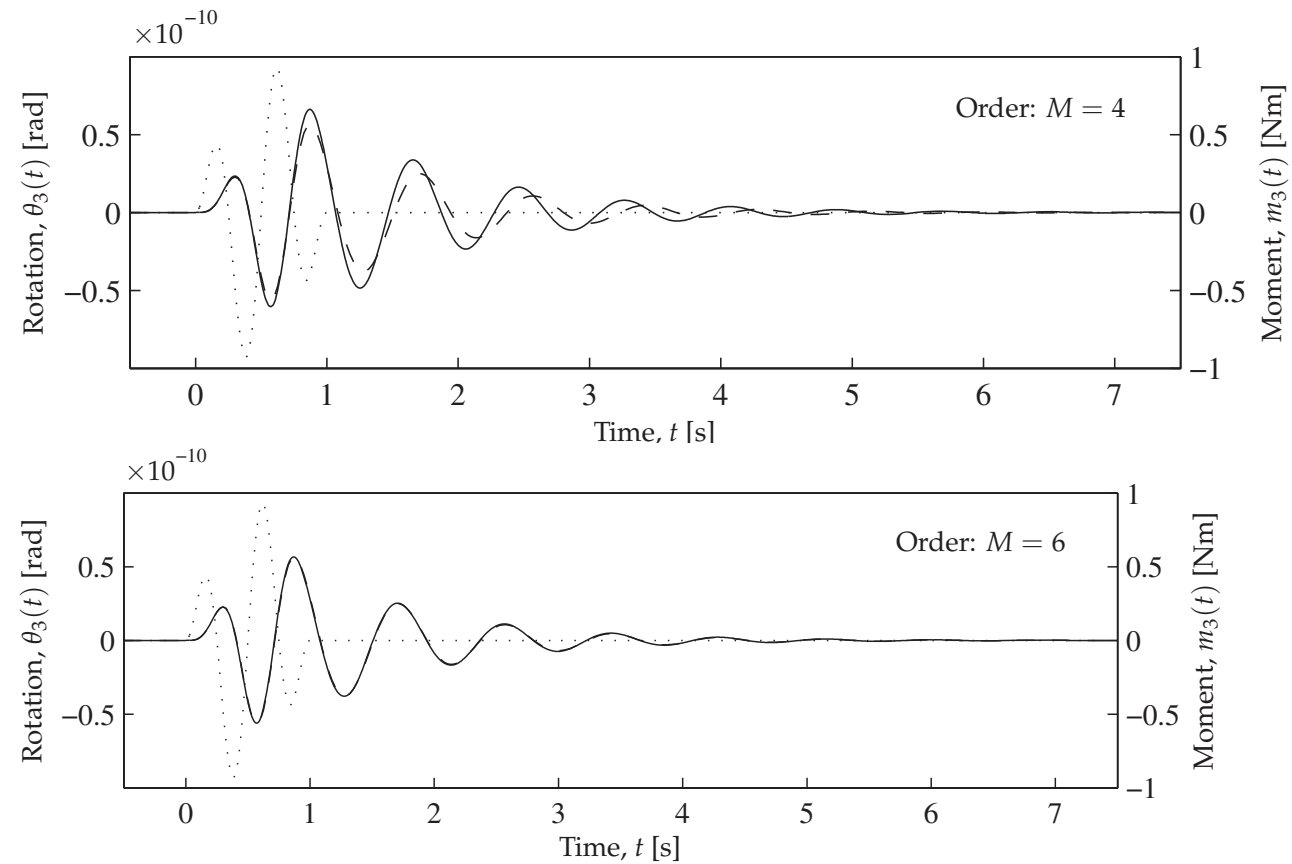

Fig. 17. Response $\theta_{3}(t)$ obtained by inverse Fourier transformation $(--)$ and lumped-parameter model ( - ). The dots $(\cdots \cdots)$ indicate the load time history. 

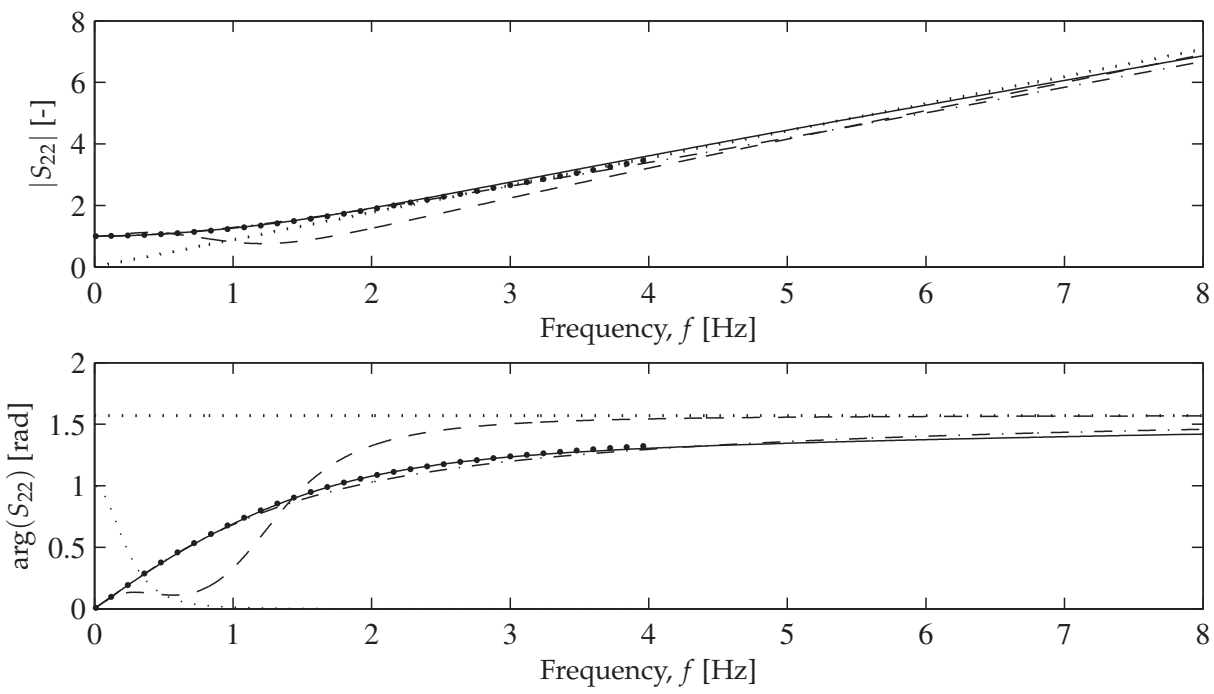

Fig. 18. Dynamic stiffness coefficient, $S_{22}$, obtained by the domain-transformation model (the large dots) and lumped-parameter models with $M=2(--), M=4(-\cdot-\cdot)$, and $M=6$ $(-)$. The thin dotted line (....$)$ ) indicates the weight function $w$ (not in radians), and the thick dotted line $(\cdots \cdots)$ indicates the high-frequency solution, i.e. the singular part of $S_{22}$.
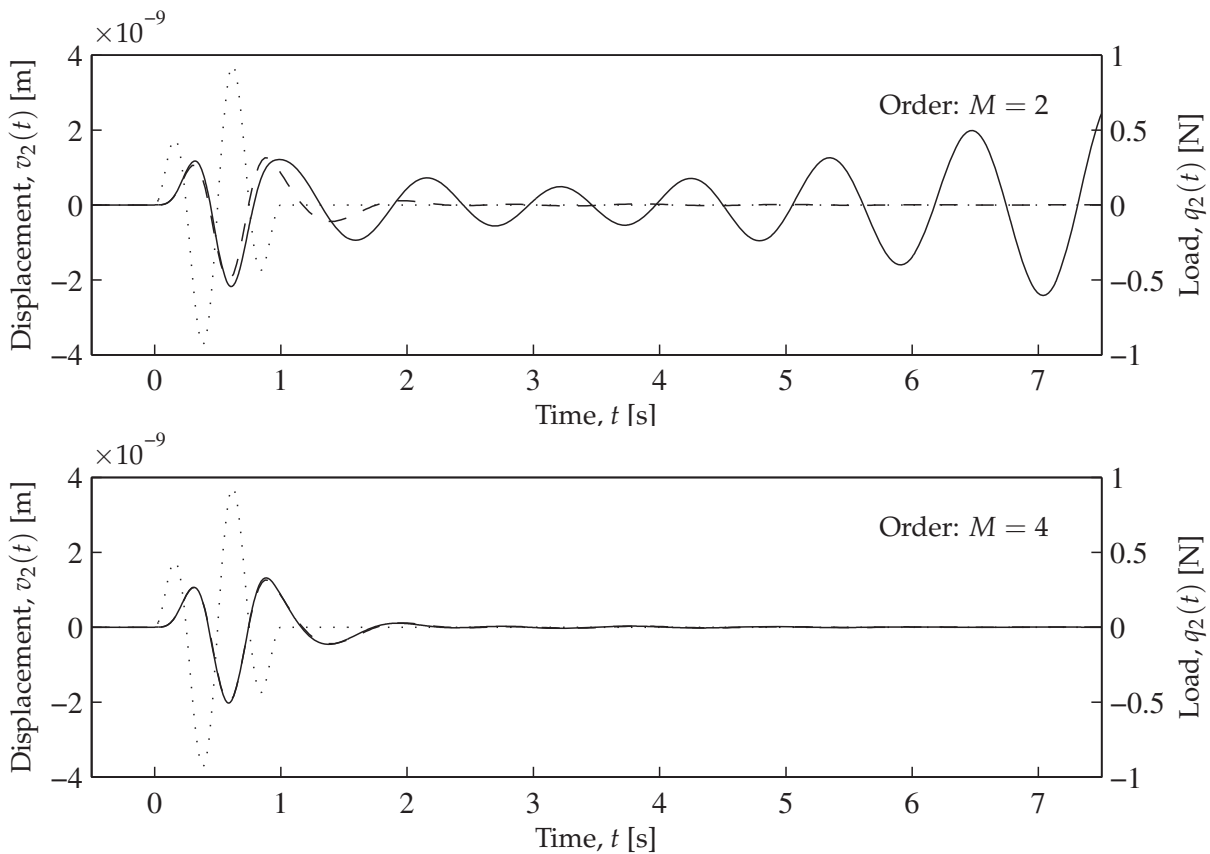

Fig. 19. Response $v_{2}(t)$ obtained by inverse Fourier transformation $(--)$ and lumped-parameter model $(-)$. The dots $(\cdots \cdots)$ indicate the load time history. 

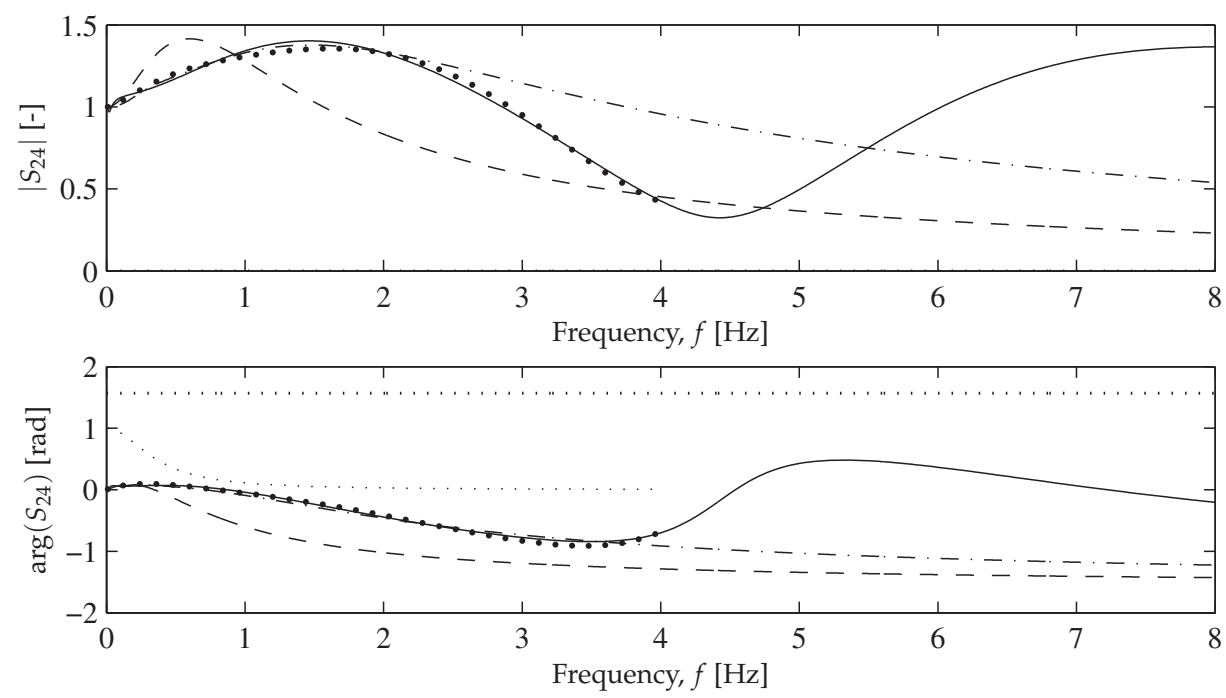

Fig. 20. Dynamic stiffness coefficient, $S_{24}$, obtained by the domain-transformation model (the large dots) and lumped-parameter models with $M=2(--), M=4(-\cdot-\cdot)$, and $M=6$ $(-)$. The thin dotted line (....$)$ ) indicates the weight function $w$ (not in radians), and the thick dotted line $(\cdots \cdots)$ indicates the high-frequency solution, i.e. the singular part of $S_{24}$.
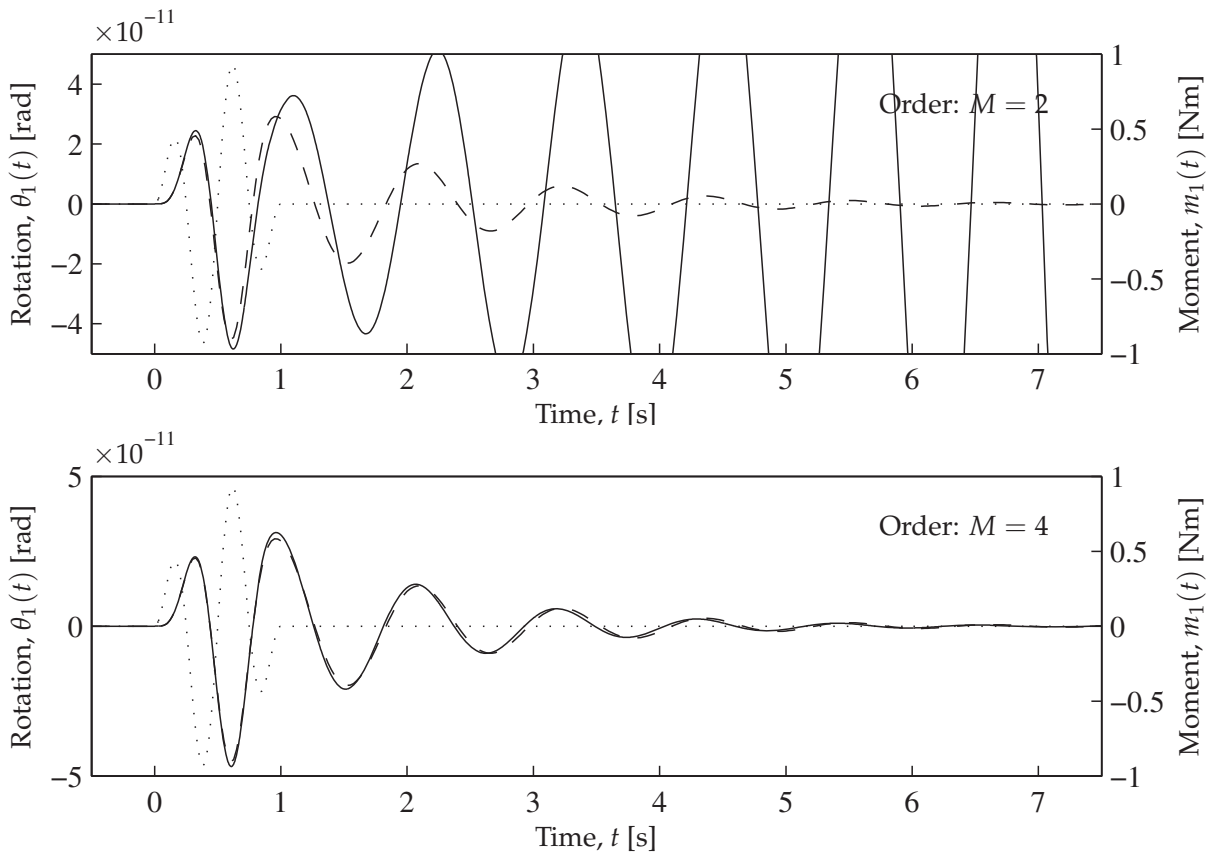

Fig. 21. Response $\theta_{1}(t)$ obtained by inverse Fourier transformation ( --$)$ and lumped-parameter model $(-)$. The dots $(\cdots \cdots)$ indicate the load time history. 

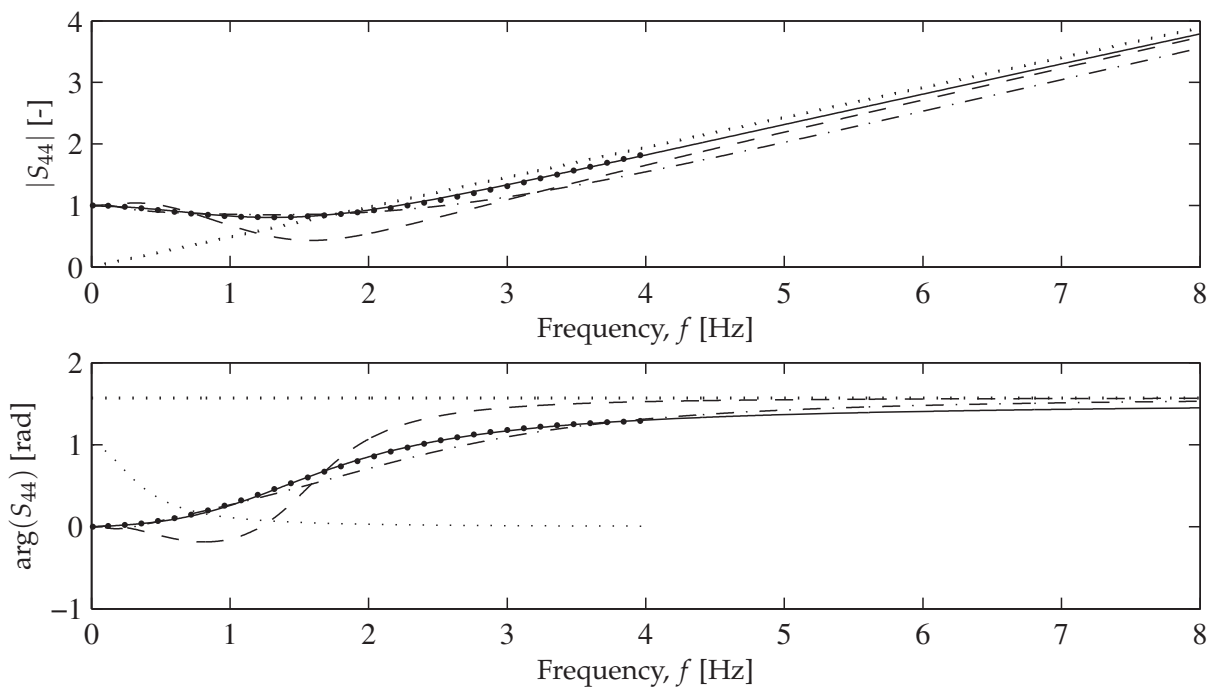

Fig. 22. Dynamic stiffness coefficient, $S_{44}$, obtained by the domain-transformation model (the large dots) and lumped-parameter models with $M=2(--), M=4(-\cdot-\cdot)$, and $M=6$ $(-)$. The thin dotted line (....$)$ ) indicates the weight function $w$ (not in radians), and the thick dotted line $(\cdots \cdots)$ indicates the high-frequency solution, i.e. the singular part of $S_{44}$.
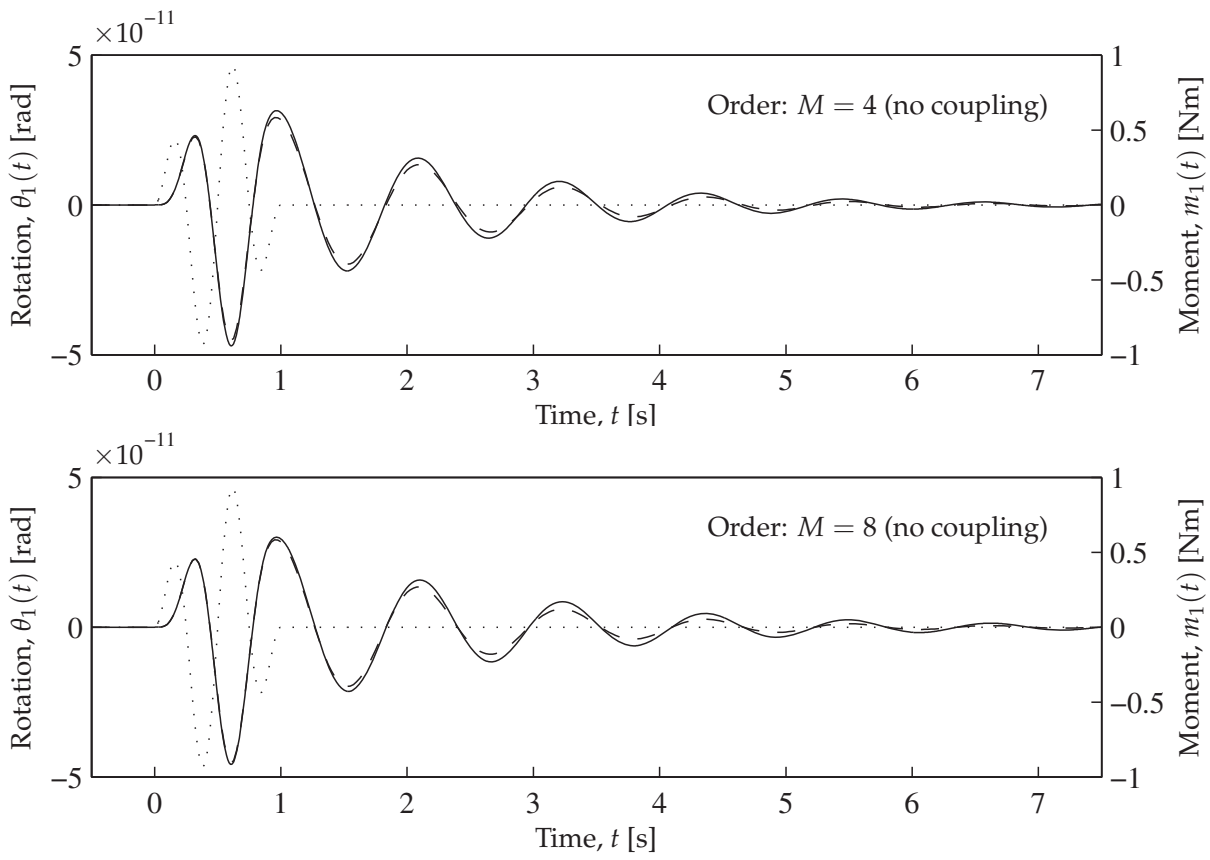

Fig. 23. Response $\theta_{1}(t)$ obtained by inverse Fourier transformation ( --$)$ and lumped-parameter model $(-)$. The dots $(\cdots \cdots)$ indicate the load time history. 
In conclusion, for the footing on the homogeneous soil it is found that an LPM with two internal degrees of freedom for the vertical and each sliding and rocking degree of freedom provides a model of great accuracy. This corresponds to fourth-order rational approximations for each of the response spectra obtained by the domain-transformation method. Little improvement is gained by including additional degrees of freedom. Furthermore, it is concluded that little accuracy is lost by neglecting the coupling between the sliding and rocking motion. However, a sixth-order model is necessary in order to get an accurate representation of the torsional impedance.

\subsubsection{Example: A footing on a layered half-space}

Next, a stratified ground is considered. The soil consists of two layers over homogeneous half-space. Material properties and layer depths are given in Table 3. This may correspond to sand over a layer of undrained clay resting on limestone or bedrock. The geometry and density of the footing are unchanged from the analysis of the homogeneous half-space.

The non-dimensional vertical and torsional impedance components, i.e. $S_{33}$ and $S_{66}$, are presented in Figs. 24 and 26 as functions of the physical frequency, $f$. In addition to the domain-transformation method results, the LPM approximations are shown for $M=2$, $M=6$ and $M=10$. Clearly, low-order lumped-parameter models are not able to describe the local tips and dips in the frequency response of a footing on a layered ground. However, the LPM with $M=10$ provides a good approximation of the vertical and torsional impedances for frequencies $f<2 \mathrm{~Hz}$. It is worthwhile to note that the lumped-parameter models of the footing on the layered ground are actually more accurate than the models of the footing on the homogeneous ground. This follows by comparison of Figs. 24 and 26 with Figs. 14 and 16. The time-domain solutions for an applied vertical force, $q_{3}(t)$, or torsional moment, $m_{3}(t)$, are plotted in Fig. 25 and Fig. 27, respectively. Evidently, the LPM with $M=6$ provides an almost exact match to the solution obtained by inverse Fourier transformation-in particular in the case of vertical motion. However, in the case of torsional motion (see Fig. 27), the model with $M=10$ is significantly better at describing the free vibration after the end of the excitation.

Next, the horizontal sliding and rocking are analysed. The non-dimensional impedance components $S_{22}, S_{24}=S_{42}$ and $S_{44}$ are shown in Figs. 28, 30 and 32 as functions of the frequency, $f$. Again, the LPM approximations with $M=2, M=6$ and $M=10$ are illustrated, and the low-order lumped-parameter models are found to be unable to describe the local variations in the frequency response. The LPM with $M=10$ provides an acceptable approximation of the sliding, the coupling and the rocking impedances for frequencies $f<$ $2 \mathrm{~Hz}$, but generally the match is not as good as in the case of vertical and torsional motion.

The transient response to a horizontal force, $q_{2}(t)$, or rocking moment, $m_{1}(t)$, are shown in Figs. 29 and 31. Again, the LPM with $M=6$ provides an almost exact match to the solution obtained by inverse Fourier transformation. However, the model with $M=10$ is significantly better at describing the free vibration after the end of the excitation. This is the case for the sliding, $v_{2}(t)$, as well as the rotation, $\theta_{1}(t)$.

\begin{tabular}{lccccc}
\hline Layer no. & $h(\mathrm{~m})$ & $E(\mathrm{MPa})$ & $v$ & $\rho\left(\mathrm{kg} / \mathrm{m}^{3}\right)$ & $\eta$ \\
\hline Layer 1 & 8 & 10 & 0.25 & 2000 & 0.03 \\
Layer 2 & 16 & 5 & 0.49 & 2200 & 0.02 \\
Half-space & $\infty$ & 100 & 0.25 & 2500 & 0.01 \\
\hline
\end{tabular}

Table 3. Material properties and layer depths for layered half-space. 

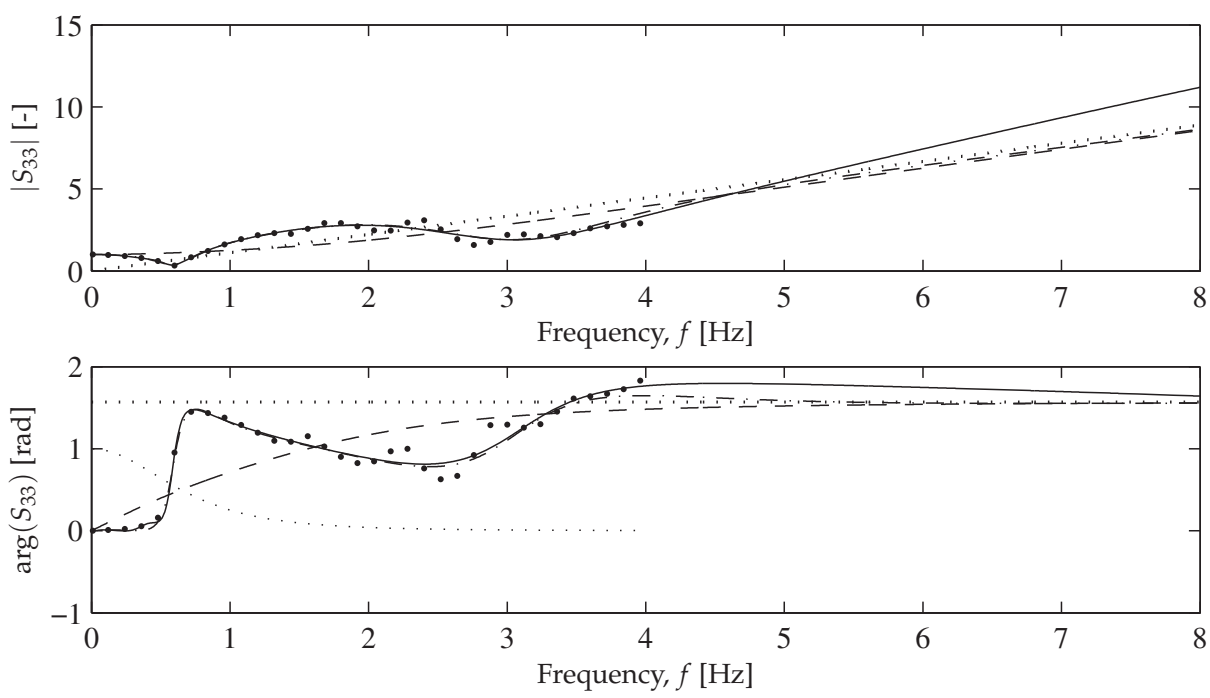

Fig. 24. Dynamic stiffness coefficient, $S_{33}$, obtained by the domain-transformation model (the large dots) and lumped-parameter models with $M=2(--), M=6(-\cdot-\cdot)$, and $M=10$ $(-)$. The thin dotted line $(\cdots . .$.$) indicates the weight function w$ (not in radians), and the thick dotted line $(\cdots \cdots)$ indicates the high-frequency solution, i.e. the singular part of $S_{33}$.
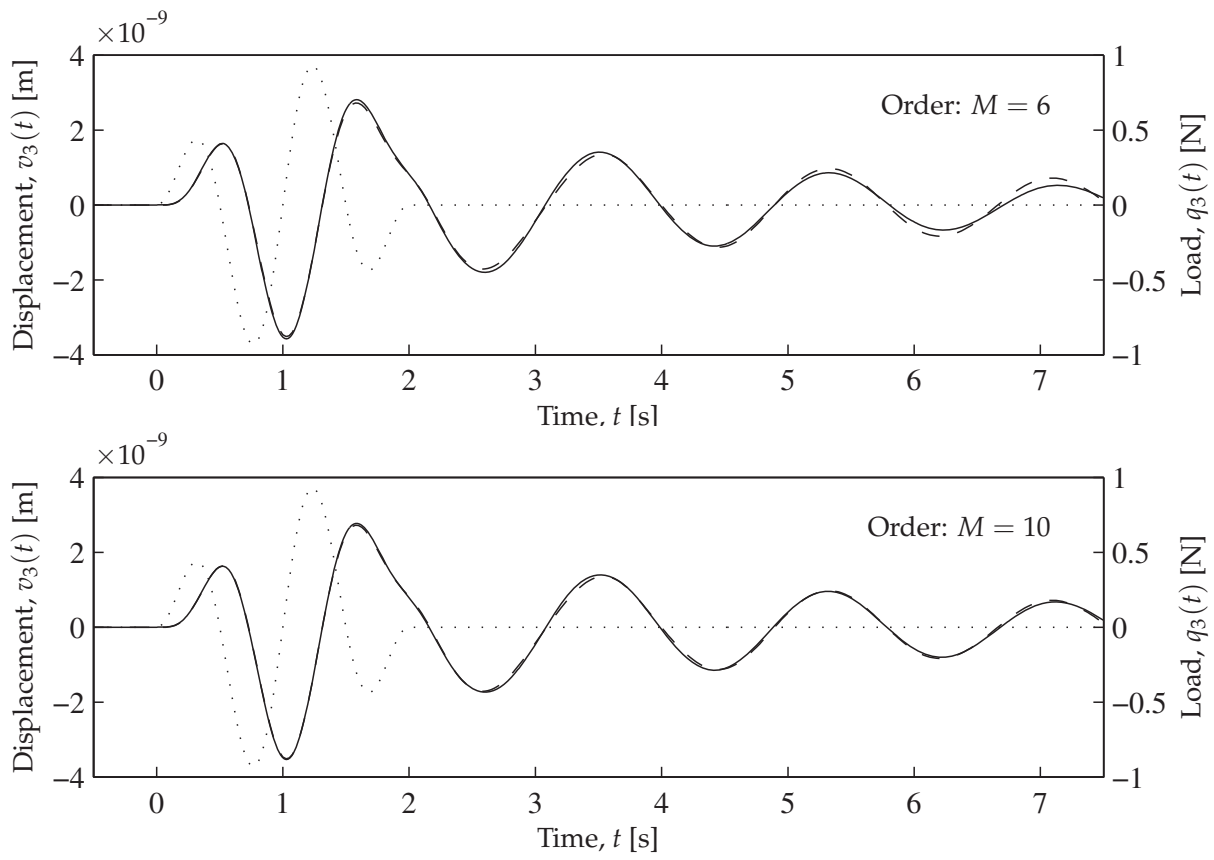

Fig. 25. Response $v_{3}(t)$ obtained by inverse Fourier transformation $(--)$ and lumped-parameter model $(-)$. The dots $(\cdots \cdots)$ indicate the load time history. 

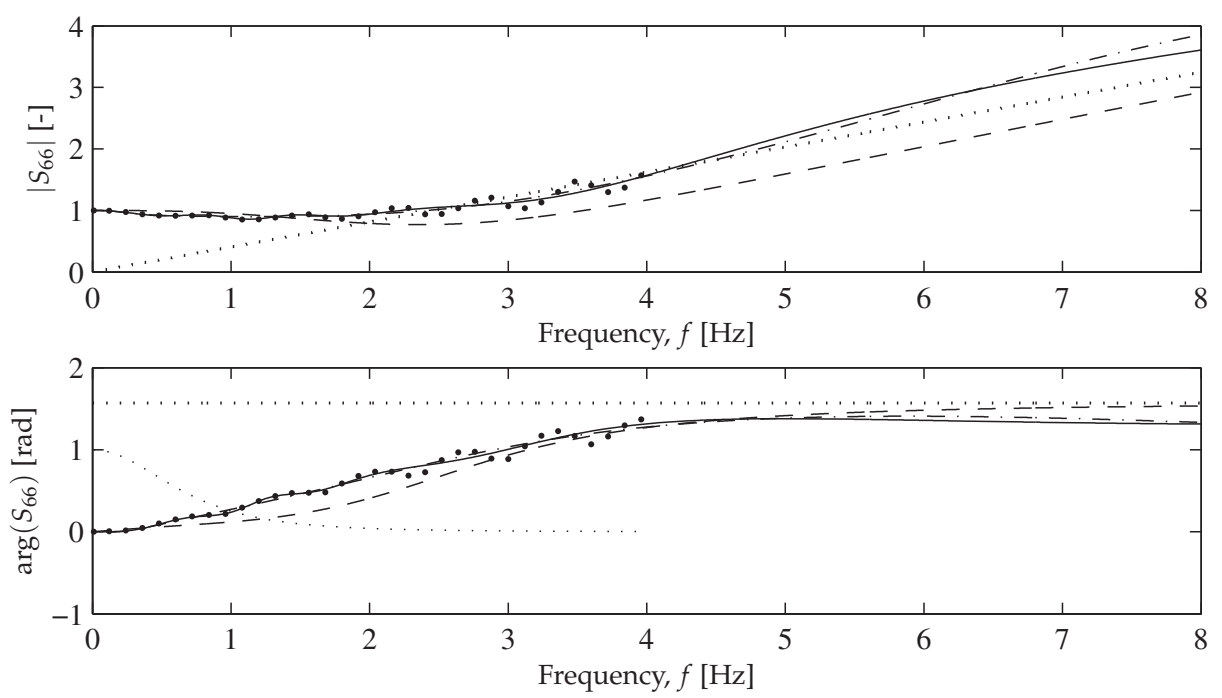

Fig. 26. Dynamic stiffness coefficient, $S_{66}$, obtained by the domain-transformation model (the large dots) and lumped-parameter models with $M=2(--), M=6(-\cdot-\cdot)$, and $M=10$ $(-)$. The thin dotted line $(\cdots . .$.$) indicates the weight function w$ (not in radians), and the thick dotted line $(\cdots \cdots)$ indicates the high-frequency solution, i.e. the singular part of $S_{66}$.
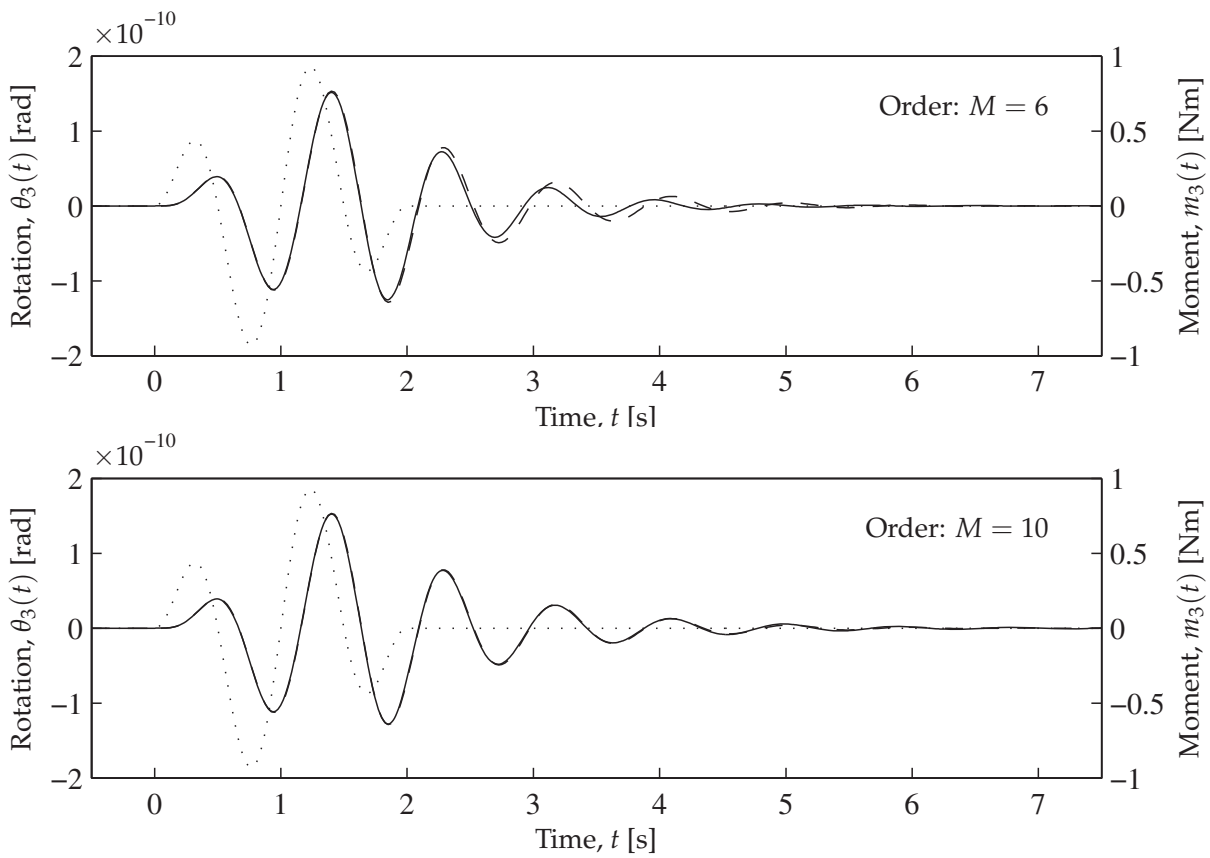

Fig. 27. Response $\theta_{3}(t)$ obtained by inverse Fourier transformation $(--)$ and lumped-parameter model ( - ). The dots $(\cdots \cdots)$ indicate the load time history. 

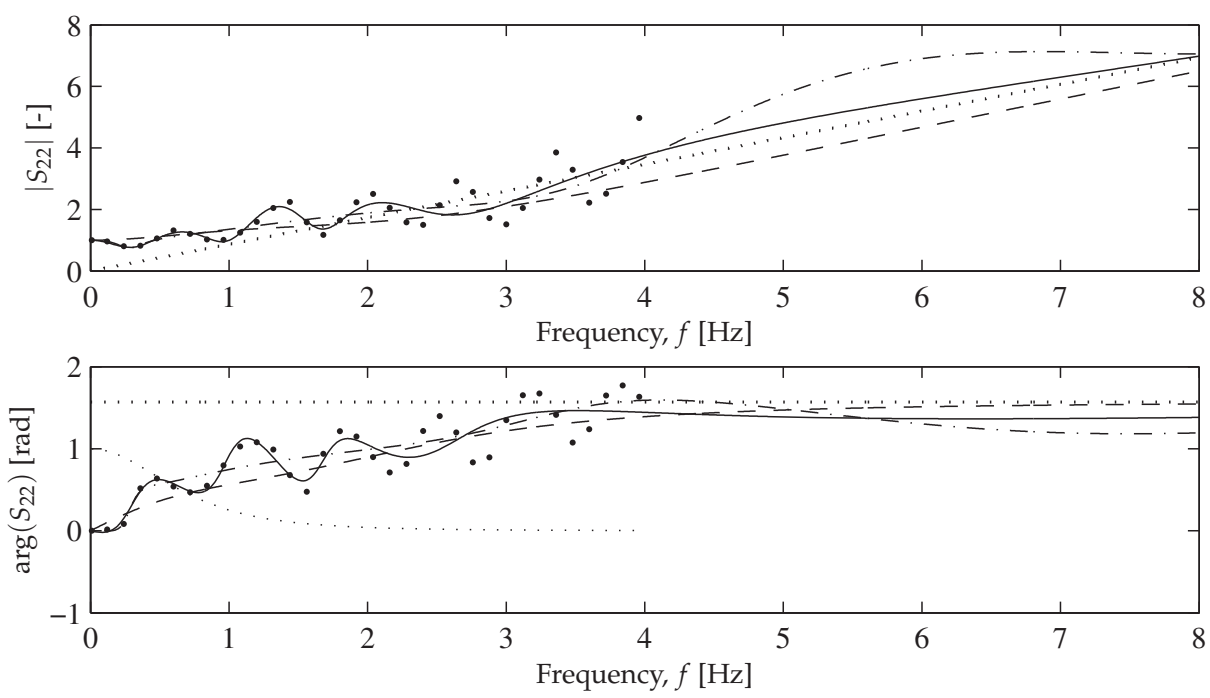

Fig. 28. Dynamic stiffness coefficient, $S_{22}$, obtained by the domain-transformation model (the large dots) and lumped-parameter models with $M=2(--), M=6(-\cdot-\cdot)$, and $M=10$ $(-)$. The thin dotted line $(\cdots . .$.$) indicates the weight function w$ (not in radians), and the thick dotted line $(\cdots \cdots)$ indicates the high-frequency solution, i.e. the singular part of $S_{22}$.
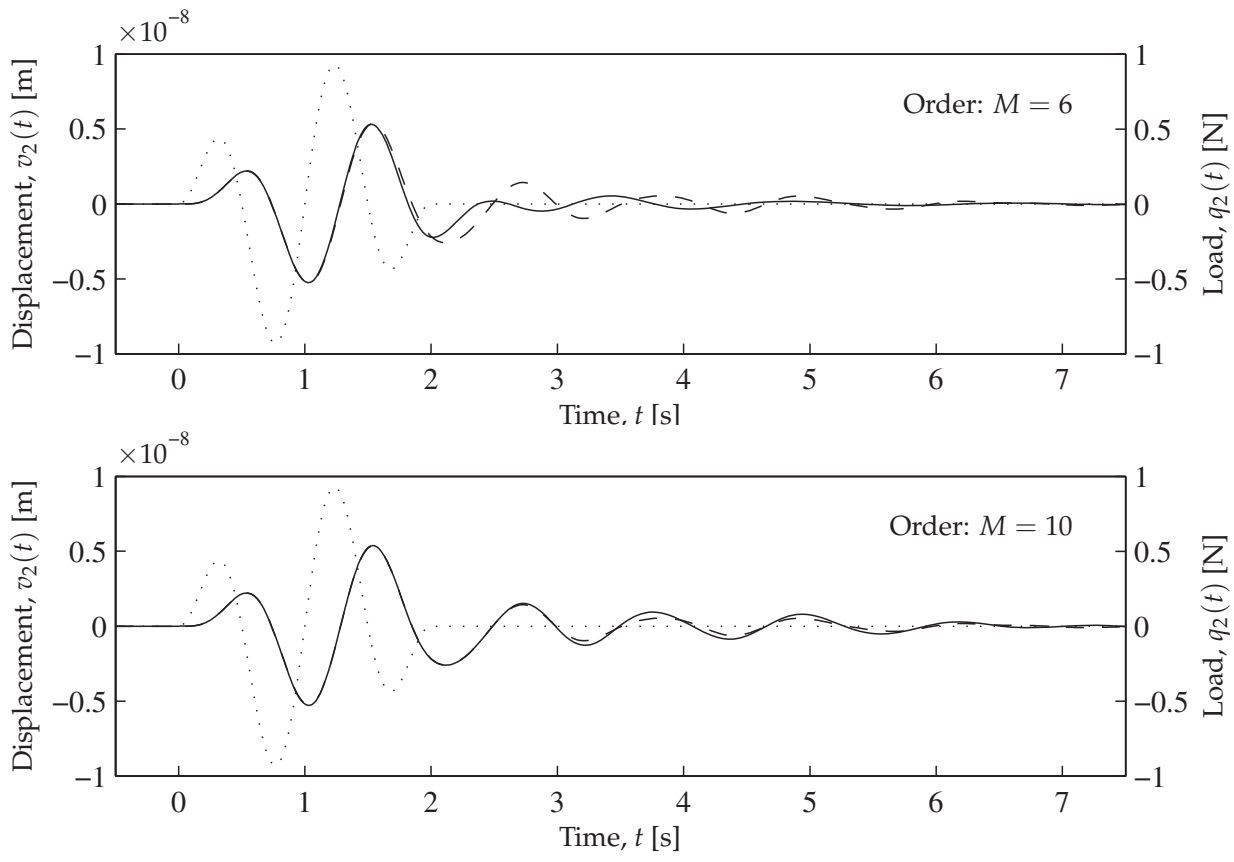

Fig. 29. Response $v_{2}(t)$ obtained by inverse Fourier transformation $(--)$ and lumped-parameter model $(-)$. The dots $(\cdots \cdots)$ indicate the load time history. 

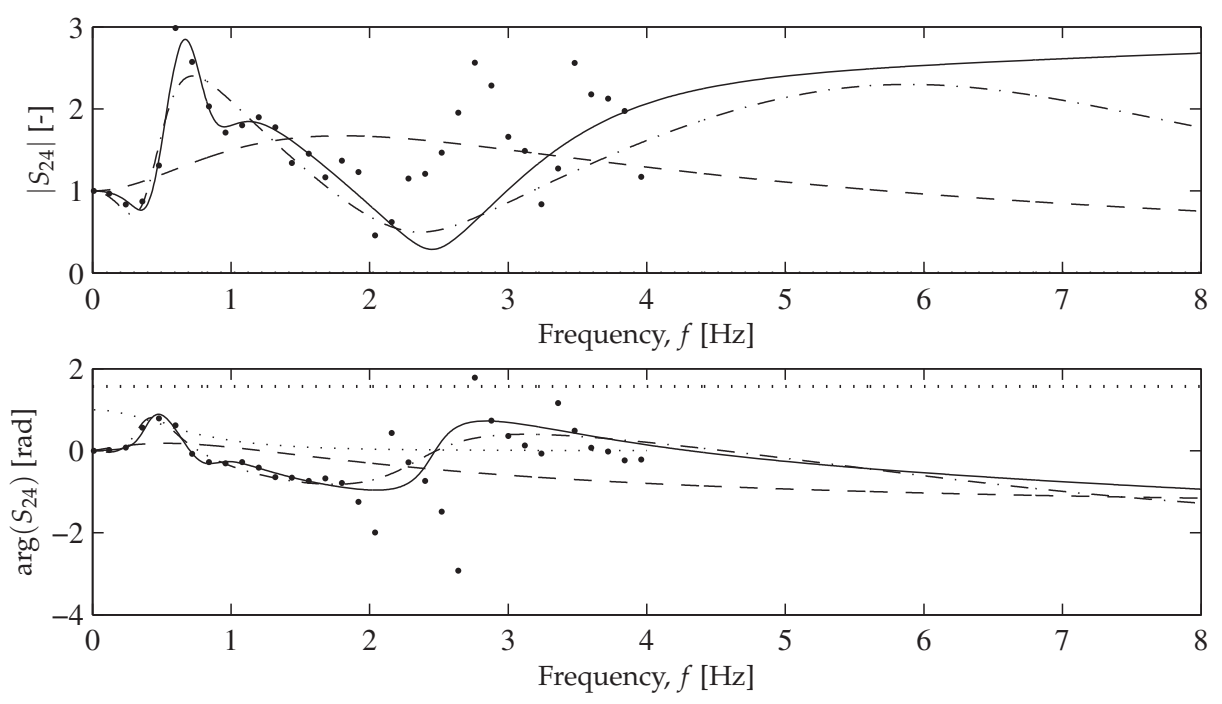

Fig. 30. Dynamic stiffness coefficient, $S_{24}$, obtained by the domain-transformation model (the large dots) and lumped-parameter models with $M=2(--), M=6(-\cdot-\cdot)$, and $M=10$ $(-)$. The thin dotted line $(\cdots \cdots)$ indicates the weight function $w$ (not in radians), and the thick dotted line $(\cdots \cdots)$ indicates the high-frequency solution, i.e. the singular part of $S_{24}$.
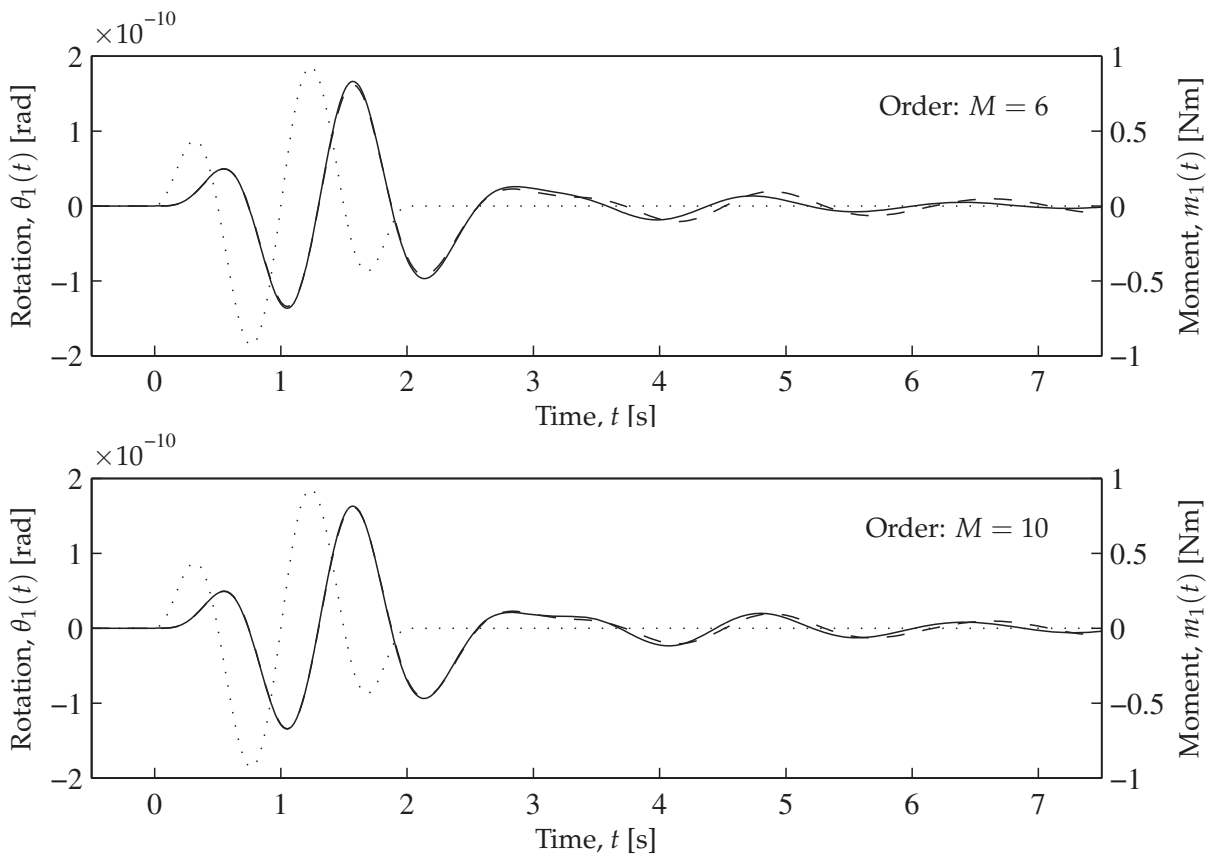

Fig. 31. Response $\theta_{1}(t)$ obtained by inverse Fourier transformation $(--)$ and lumped-parameter model ( - ). The dots $(\cdots \cdots)$ indicate the load time history. 

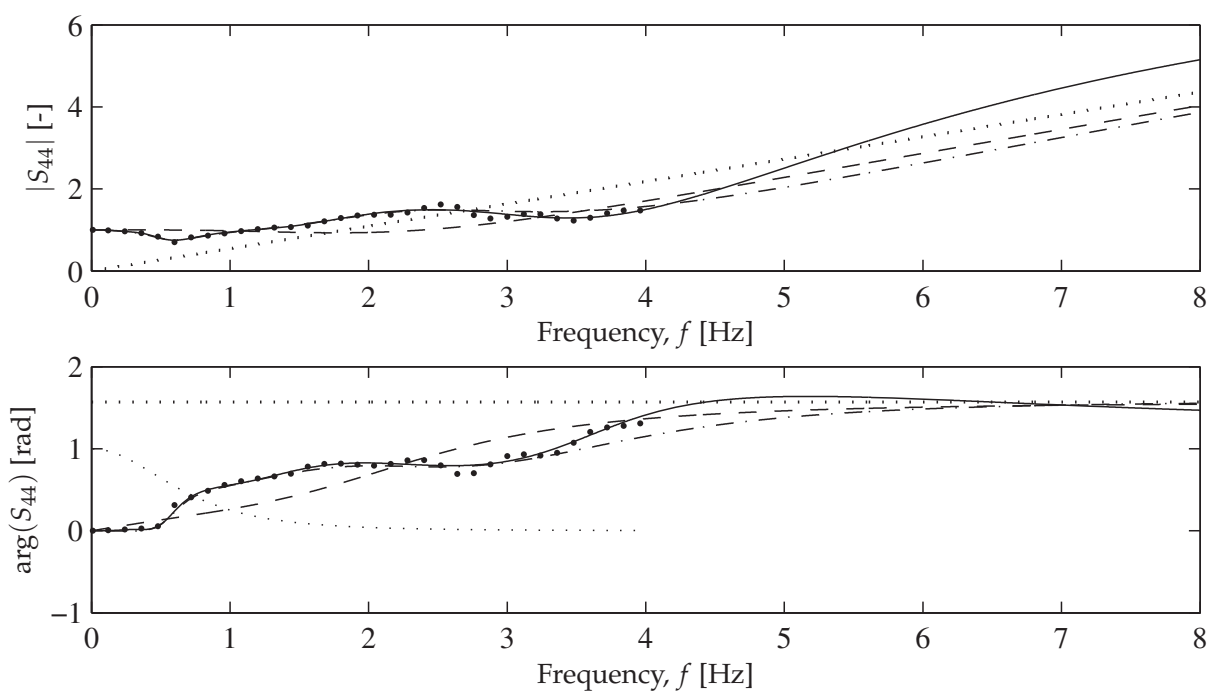

Fig. 32. Dynamic stiffness coefficient, $S_{44}$, obtained by the domain-transformation model (the large dots) and lumped-parameter models with $M=2(--), M=6(-\cdot-\cdot)$, and $M=10$ ( - ). The thin dotted line (.....$)$ indicates the weight function $w$ (not in radians), and the thick dotted line $(\cdots \cdots)$ indicates the high-frequency solution, i.e. the singular part of $S_{44}$.
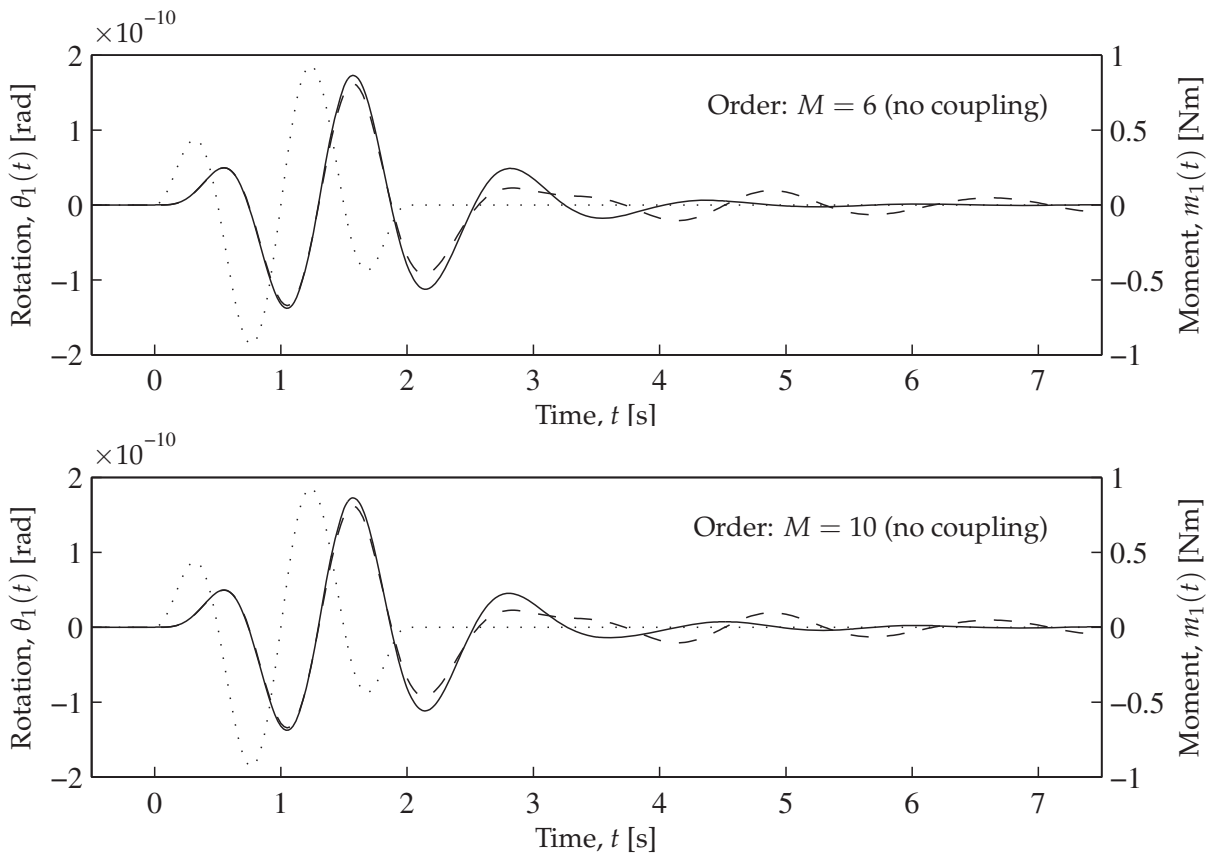

Fig. 33. Response $\theta_{1}(t)$ obtained by inverse Fourier transformation ( --$)$ and lumped-parameter model $(-)$. The dots $(\cdots \cdots)$ indicate the load time history. 
Finally, in Fig. 33 the results are given for the alternative LPM, in which the coupling between sliding and rocking has been neglected. It is observed that the maximum response occurring during loading is predicted with almost the same accuracy as by the model in which the coupling is accounted for. However, the geometrical damping is badly described with regard to the decrease in magnitude and, in particular, the phase of the response during the free vibration. Hence, the response of the footing on the layered ground cannot be predicted with low order models, and an LPM with 3-5 internal degrees of freedom is necessary for each nonzero term in the impedance matrix, i.e. rational approximations of the order 6-10 are required. In particular, it is noted that the impedance term providing the coupling between sliding and rocking is not easily fitted by an LPM of low order, i.e. orders below six. The maximum response is well predicted without the coupling term; however, if the coupling is not accounted for, the geometrical damping is poorly described. This may lead to erroneous conclusions regarding the fatigue lifespan of structures exposed to multiple transient dynamic loads, e.g. offshore wind turbines.

\subsection{Example: A flexible foundation embedded in viscoelastic soil}

In this example, a skirted circular foundation, also known as a bucket foundation, is analysed using the coupled finite-element method (FEM) and boundary-element method (BEM). The bucket has the radius $r_{0}=10 \mathrm{~m}$ and the skirt length $h_{0}=12 \mathrm{~m}$, see Fig. 34 . The lid has a thickness of $t_{\text {lid }}=0.50 \mathrm{~m}$, whereas the thickness of the skirt is $t_{\text {skirt }}=50 \mathrm{~mm}$. The bucket consists of steel, and the material properties are given in Table 4. The model of the lid is unrealistic and the real structure may be lighter and stiffer than the solid plate but with a complex geometry not easily modelled.

\begin{tabular}{lcccc}
\hline Material & $E(\mathrm{MPa})$ & $v$ & $\rho\left(\mathrm{kg} / \mathrm{m}^{3}\right)$ & $\eta$ \\
\hline Soil & 20 & 0.25 & 2000 & 0.03 \\
Steel (bucket) & 200.000 & 0.30 & 7850 & 0.01 \\
\hline
\end{tabular}

Table 4. Material properties of the bucket foundation and the subsoil.
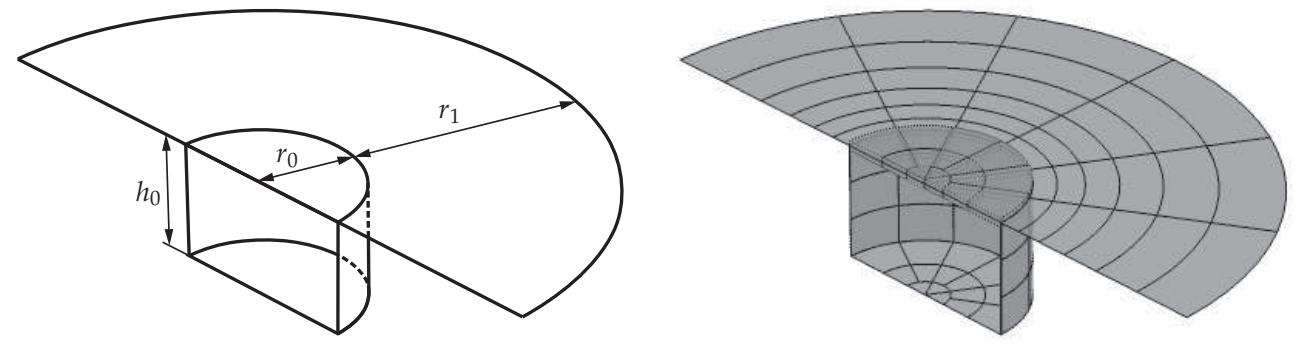

Fig. 34. Coupled finite-element/boundary-element model of skirted foundation: Geometry (left) and discretization (right). Only half the foundation is discretized, utilising the symmetry of the problem. 
A thorough introduction to the FEM and BEM is beyond the scope of the present text. The reader is referred to the text books by Bathe (1996) and Petyt (1998) for a detailed explanation of the FEM, whereas information about the BEM can be found, for example, in the work by Brebbia (1982) and Domínguez (1993). The coupling of BEM and FEM schemes has been discussed by, among others, Mustoe (1980), von Estorff \& Kausel (1989), Stamos \& Beskos (1995), Jones et al. (1999), Elleithy et al. (2001) and Andersen (2002).

In the present example, a closed boundary-element (BE) domain is applied for the soil inside the bucket and an open BE domain is utilised for the remaining half-space. In either case, quadratic spatial interpolation is applied of the displacement and traction fields on the boundaries discretized by boundary elements, i.e. elements with nine nodes are applied. Finally, the lid and the skirt of the bucket foundation are discretized using shell finite elements. Again, quadrilaterals with nine nodes are used.

With $\rho, c_{P}$ and $c_{S}$ denoting the mass density, the P-wave velocity and the S-wave velocity of the soil, respectively, the high-frequency limit of the impedance components are given as

$$
\begin{gathered}
c_{33}^{\infty}=\rho c_{P} A_{\text {lid }}+\rho c_{S} A_{\text {skirt }}, \\
c_{66}^{\infty}=2 \rho c_{S} \mathcal{I}_{\text {lid }}+\rho c_{S} A_{\text {skirt }} r_{0}^{2}, \\
c_{22}^{\infty}=\rho c_{S} A_{\text {lid }}+\frac{1}{2}\left(\rho c_{S}+\rho c_{P}\right) A_{\text {skirt }}, \\
c_{24}^{\infty}=c_{42}^{\infty}=-\frac{1}{2}\left(\rho c_{S}+\rho c_{P}\right) A_{\text {skirt }} \frac{h_{0}}{2}=-c_{15}^{\infty}=-c_{51}^{\infty}, \\
c_{44}^{\infty}=c_{55}^{\infty}=\rho c_{P} \mathcal{I}_{\text {lid }}+\frac{1}{2}\left(\rho c_{S}+\rho c_{P}\right) \mathcal{I}_{\text {skirt }}+\frac{1}{2} \rho c_{S} A_{\text {skirt }} r_{0}^{2},
\end{gathered}
$$

where $A_{\text {lid }}=\pi r_{0}^{2}$ and $A_{\text {skirt }}=4 \pi r_{0} h_{0}$ are the areas of the lid and the skirt, respectively. The latter accounts for the inside as well as the outside of the skirt; hence, the factor 4 instead of the usual factor 2 . Further, $\mathcal{I}_{\text {lid }}$ and $\mathcal{I}_{\text {skirt }}$ are the geometrical moments of inertia of the lid and the skirt around the centroid of the lid, defined as

$$
\mathcal{I}_{\text {lid }}=\frac{\pi}{4} r_{0}^{4}, \quad \mathcal{I}_{\text {skirt }}=\frac{4 \pi}{3} r_{0} h_{0}^{3} .
$$

Again, the contributions from both sides of the skirt are included and it is noted that the torsional moment of inertia of the lid is simply $2 \mathcal{I}_{\text {lid }}$.

In the following, an explanation is given of the terms in Eq. (109). Firstly, the vertical impedance is given as the sum of a contribution from the P-waves emanating from the bottom of the lid, represented by the first term in Eq. (109a), and a second contribution from the S-waves produced at the exterior and interior surfaces of the skirt when the foundation moves up and down as a rigid body. This may be an overestimation of the impedance for a flexible foundation and alternatively a high-frequency solution with no contributions from the skirt may be proposed.

Secondly, the torsional impedance provided by Eq. (109b) contains a contribution from the $\mathrm{S}$-waves generated at the bottom of the lid, whereas the second term represents the S-waves stemming from the rotation of the skirt around the vertical axis. Thirdly, the horizontal impedance given by Eq. (109c) is composed of three terms. The first term represent S-waves initiated at the bottom of the lid, whereas the second term is due to the S- and P-waves arising at the skirt. Only S-waves are generated at the vertical lines at the "sides" of the foundation, and only P-waves are produced at the vertical lines on the "back" and "front" 

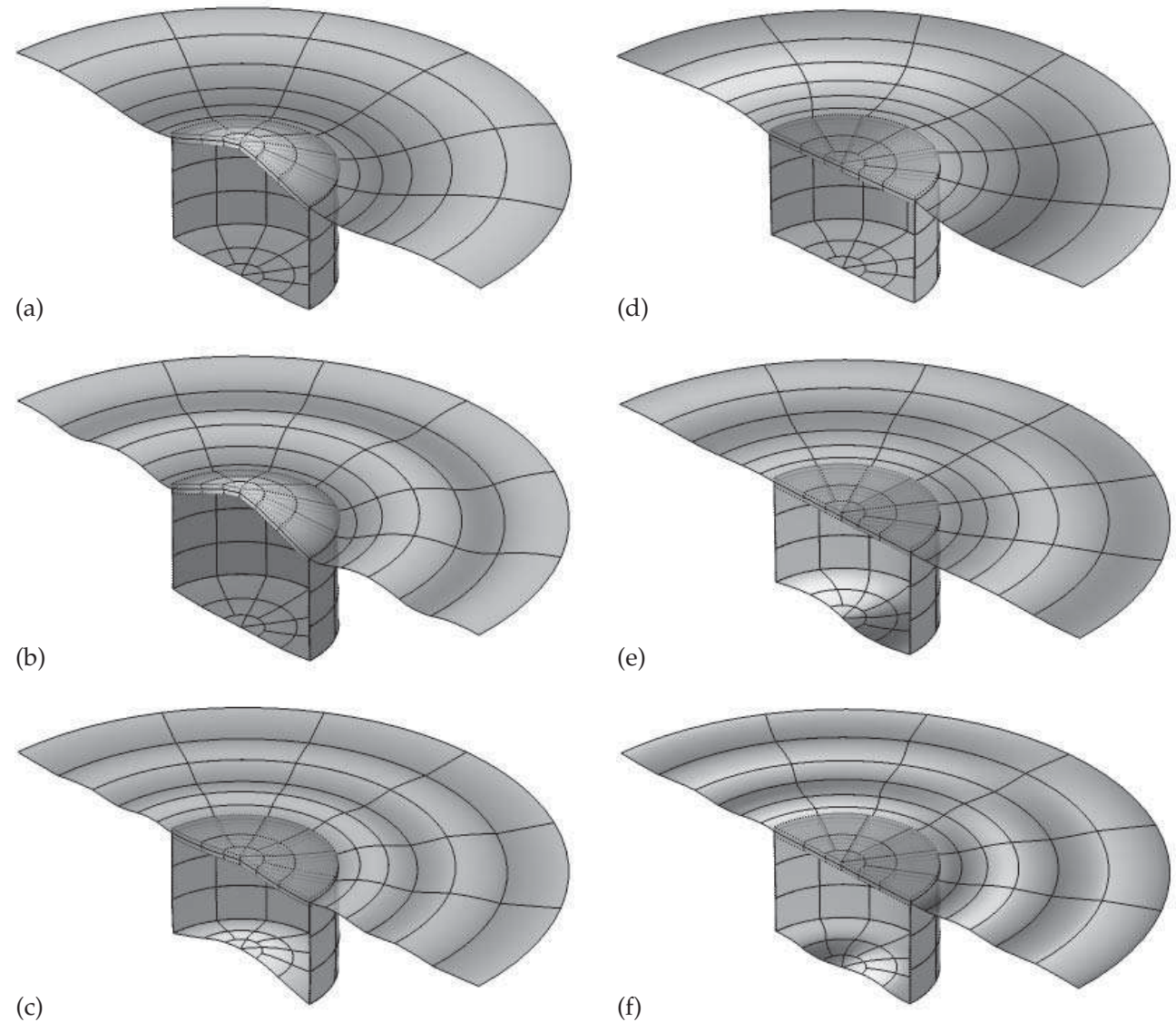

Fig. 35. Response in phase with the load for the skirted foundation. On the left, the results are shown for a unit-magnitude harmonic vertical force acting at the centre of the foundation at the frequencies (a) $f=2 \mathrm{~Hz}$, (b) $f=4 \mathrm{~Hz}$ and (c) $f=6 \mathrm{~Hz}$. The displacements are scaled by a factor of $10^{9}$ and the light and dark shades of grey indicate positive and negative vertical displacements. On the right, the results are shown for a unit-magnitude harmonic torsional moment acting around the centre of the foundation at the frequencies (a) $f=2 \mathrm{~Hz}$, (b) $f=4 \mathrm{~Hz}$ and (c) $f=6 \mathrm{~Hz}$. The displacements are scaled by a factor of $10^{10}$ and the light and dark shades of grey indicate positive and negative displacements in the direction orthogonal to the plane of antisymmetry.

of the bucket. However, at all other vertical lines, a combination of P- and S-waves are emitted. A formal mathematical proof of Eq. (109c) follows by integration along the perimeter of the skirt. However, by physical reasoning one finds that half the area $A_{\text {skirt }}$ emits P-waves, concentrating around the "back" and "front" of the foundation, whereas the other half of $A_{\text {skirt }}$, i.e. the "sides", emits S-waves. A similar reasoning lies behind the derivation of $c_{24}^{\infty}$, and as indicated by Eq. (109d) there are no contributions from the lid to the sliding-rocking coupling at high frequencies. 

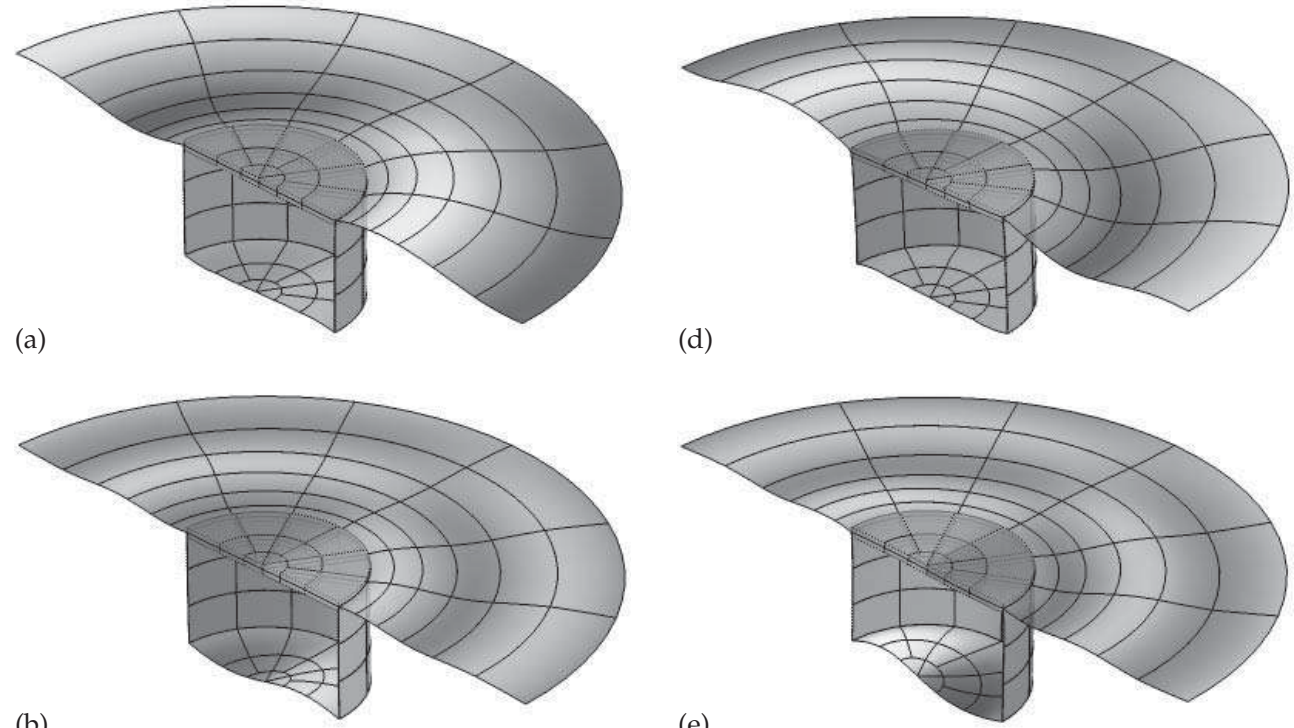

(b)
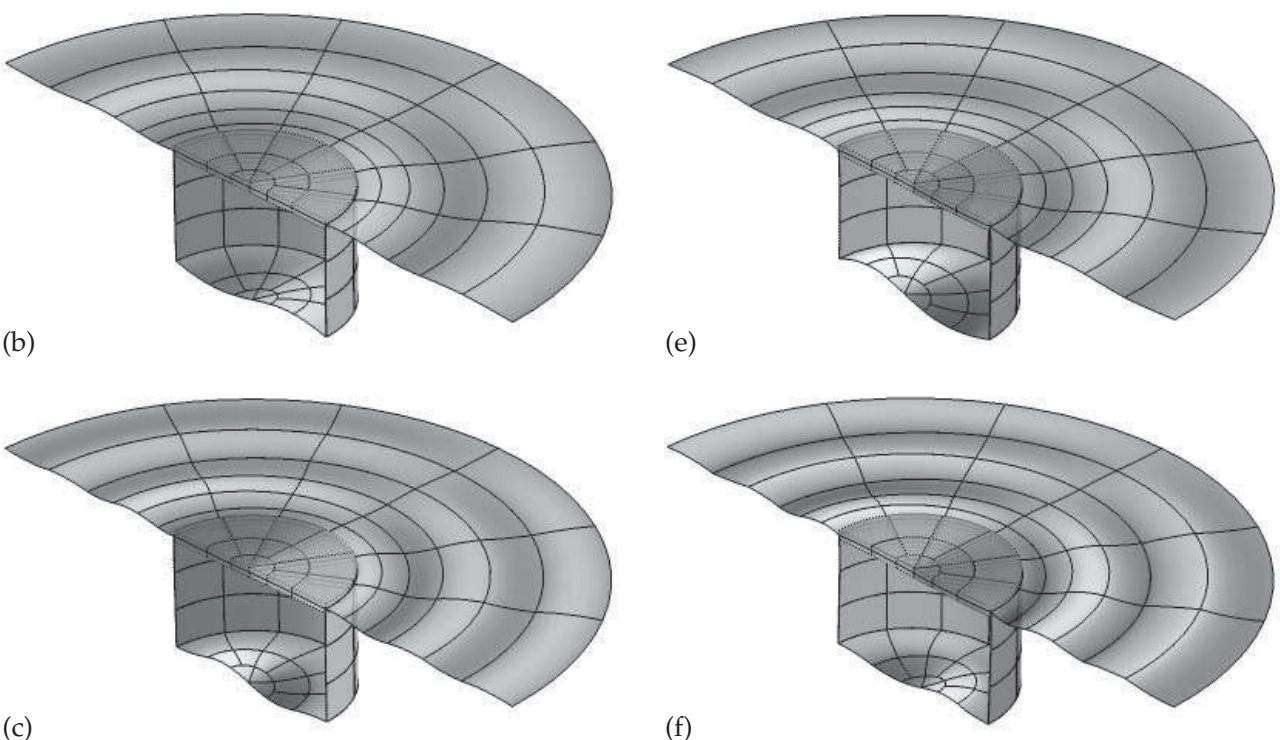

Fig. 36. Response in phase with the load for the skirted foundation. On the left, the results are shown for a unit-magnitude harmonic horizontal force acting at the centre of the foundation at the frequencies (a) $f=2 \mathrm{~Hz}$, (b) $f=4 \mathrm{~Hz}$ and (c) $f=6 \mathrm{~Hz}$. The displacements are scaled by a factor of $10^{9}$ and the light and dark shades of grey indicate positive and negative vertical displacements. On the right, the results are shown for a unit-magnitude harmonic rocking moment acting around the centre of the foundation at the frequencies (a) $f=2 \mathrm{~Hz}$, (b) $f=4 \mathrm{~Hz}$ and (c) $f=6 \mathrm{~Hz}$. The displacements are scaled by a factor of $10^{10}$ and the light and dark shades of grey indicate positive and negative vertical displacements.

Finally, the rocking impedance provided by Eq. (109e) consists of three parts. The first one stems from $\mathrm{P}$-waves originating from the bottom of the lid and the next term is a mixture of $\mathrm{P}$ - and $\mathrm{S}$-waves generated at the skirts. However, only the S-waves polarised in the horizontal direction are included in the second term of Eq. (109e); but the rocking motion of the foundation also induces S-waves polarised in the vertical direction, in particular at the "back" and "front" of the bucket. Again, a strict proof follows by integration over the surface of the skirts, but a by physical reasoning it is found the half the area contributes to the generation of such S-waves. 
The response of the bucket foundation is computed at 31 discrete frequencies from 0 to $6 \mathrm{~Hz}$ using the coupled boundary-element-finite-element model. The results for vertical and torsional excitation and three different frequencies are shown in Fig. 35, whereas the corresponding results for horizontal sliding and rocking are given in Fig. 36. It is noted that the light and dark shades of grey indicate vertical displacements upwards and downwards, respectively, in the plots for the vertical, horizontal and rocking-moment excitation. However, in the case of torsional excitation, no vertical displacements are generated. Hence, in this particular case the light/dark shades indicate horizontal displacements away from/towards the plane of symmetry.

Figures 37 to 40 show the frequency-domain solution obtained by the coupled finite-element-boundary-element scheme for the different impedance components, excluding the vertical impedance. The results of the corresponding lumped-parameter models of orders 2, 6 and 10 are plotted in the same figures. Clearly, the low-order models with $M=2$, and having only a single internal degree-of-freedom, are inadequate. A similar conclusion can be made for the vertical impedance that is not shown here. However, the sixth-order lumped-parameter model provide a good fit to the FE-BE model in the low-frequency range and only an insignificant improvement is obtained by increasing the order of the LPMs to 10. It is of particular interest that the high-order LPM with $M=10$ does not lead to a better fit at higher frequencies than the sixth-order model. Instead, wiggling occurs at low-frequencies with the tenth-order model, i.e. non-physical tips and dips arise in the results from the LPM between frequencies at which the target FE-BE solution has been computed. This behaviour should be avoided and thus the sixth-order model is preferred. In order to obtain a better fit without wiggling, a smaller frequency step should be applied in the FE-BE solution.
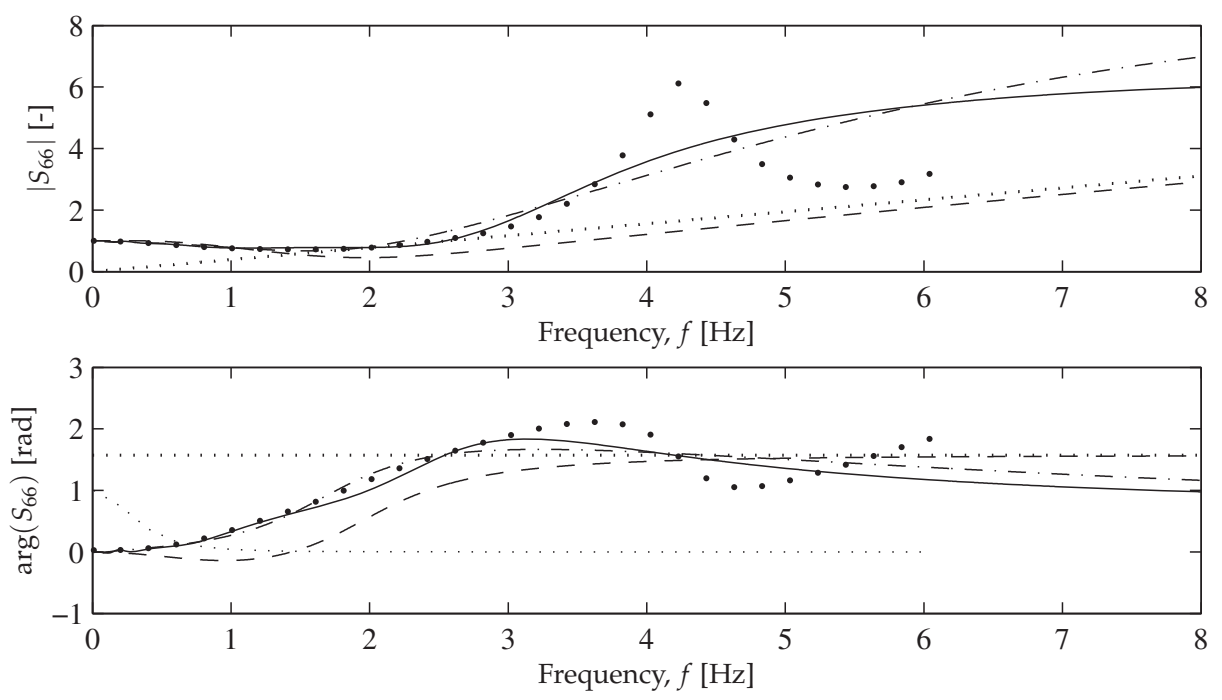

Fig. 37. Dynamic stiffness coefficient, $S_{66}$, obtained by finite-element-boundary-element (the large dots) and lumped-parameter models with $M=2(--), M=6(-\cdot-\cdot)$, and $M=10$ $(-)$. The thin dotted line ( $. \cdots .$.$) indicates the weight function w$ (not in radians), and the thick dotted line $(\cdots \cdots)$ indicates the high-frequency solution, i.e. the singular part of $S_{66}$. 

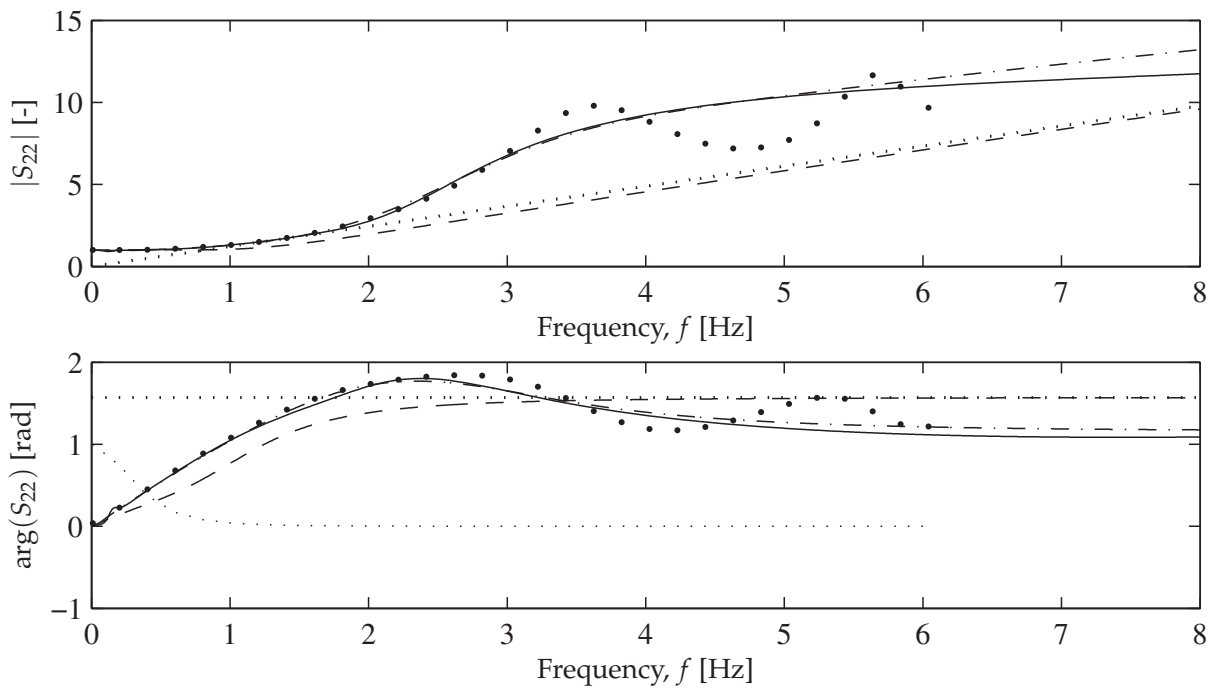

Fig. 38. Dynamic stiffness coefficient, $S_{22}$, obtained by finite-element-boundary-element (the large dots) and lumped-parameter models with $M=2(--), M=6(-\cdot-\cdot)$, and $M=10$ $(-)$. The thin dotted line (....$)$ ) indicates the weight function $w$ (not in radians), and the thick dotted line $(\cdots \cdots)$ indicates the high-frequency solution, i.e. the singular part of $S_{22}$.
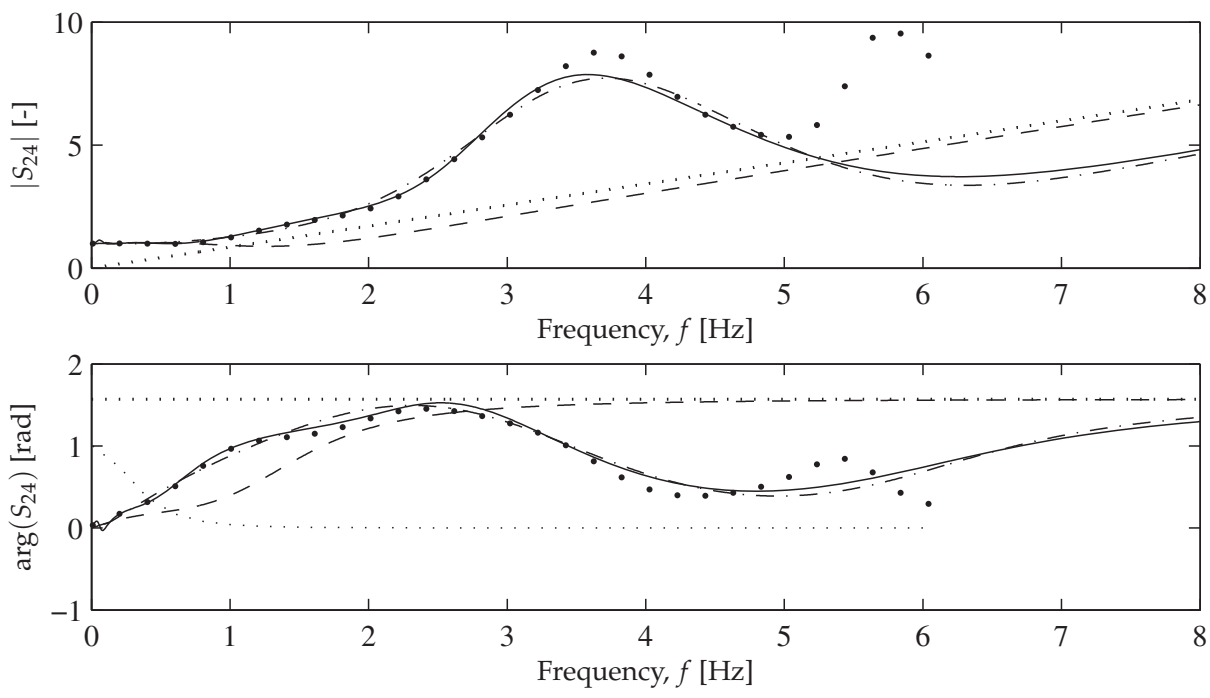

Fig. 39. Dynamic stiffness coefficient, $S_{24}$, obtained by finite-element-boundary-element (the large dots) and lumped-parameter models with $M=2(--), M=6(-\cdot-\cdot)$, and $M=10$ $(-)$. The thin dotted line ( $\ldots . .$.$) ) indicates the weight function w$ (not in radians), and the thick dotted line $(\cdots \cdots)$ ) indicates the high-frequency solution, i.e. the singular part of $S_{24}$. 

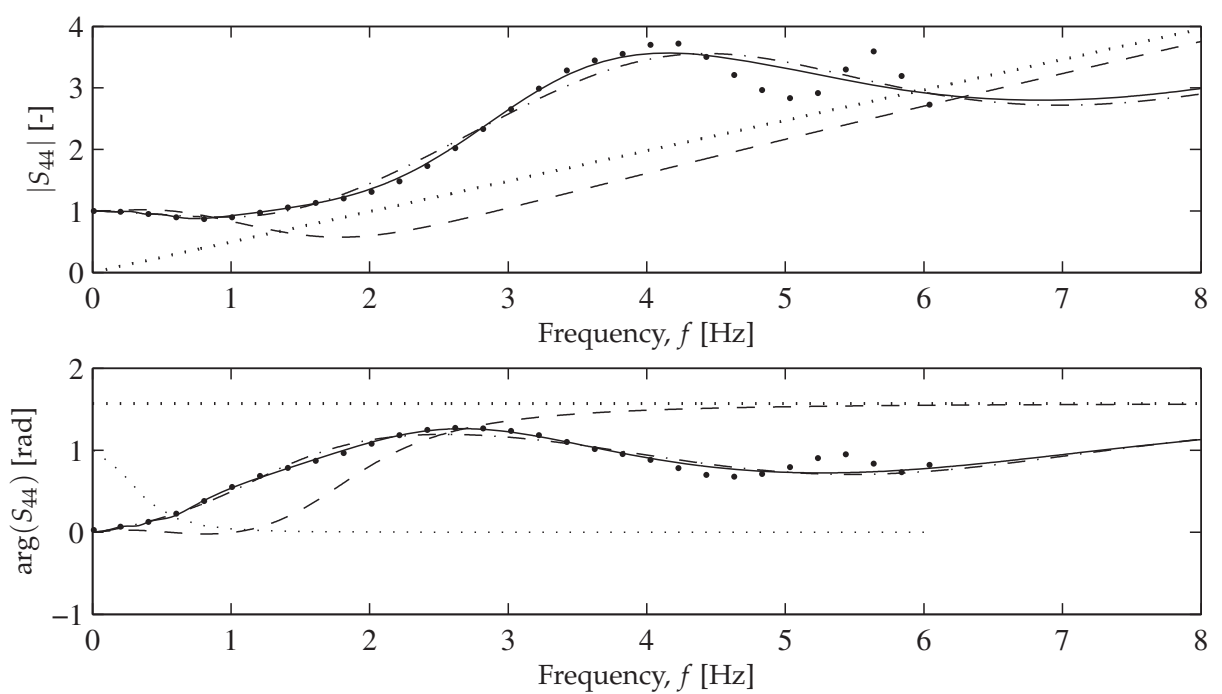

Fig. 40. Dynamic stiffness coefficient, $S_{44}$, obtained by domain-transformation (the large dots) and lumped-parameter models with $M=2(--), M=6(-\cdot-\cdot)$, and $M=10$ ( — $)$. The thin dotted line ( $. \cdots .$.$) indicates the weight function w$ (not in radians), and the thick dotted line ( $\cdots \cdots$ ) indicates the high-frequency solution, i.e. the singular part of $S_{44}$.

\section{Summary}

This chapter discusses the formulation of computational models that can be used for an efficient analysis of wind turbine foundations. The purpose is to allow the introduction of a foundation model into aero-elastic codes without a dramatic increase in the number of degrees of freedom in the model. This may be of particular interest for the determination of the fatigue life of a wind turbine.

After a brief introduction to different types of foundations for wind turbines, the particular case of a rigid footing on a layered ground is treated. A formulation based on the so-called domain-transformation method is given, and the dynamic stiffness (or impedance) of the foundation is calculated in the frequency domain. The method relies on an analytical solution for the wave propagation over depth, and this provides a much faster evaluation of the response to a load on the surface of the ground than may be achieved with the finite element method and other numerical methods. However, the horizontal wavenumber-frequency domain model is confined to the analysis of strata with horizontal interfaces.

Subsequently, the concept of a consistent lumped-parameter model (LPM) has been presented. The basic idea is to adapt a simple mechanical system with few degrees of freedom to the response of a much more complex system, in this case a wind turbine foundation interacting with the subsoil. The use of a consistent LPM involves the following steps:

1. The target solution in the frequency domain is computed by a rigorous model, e.g. a finite-element or boundary-element model. Alternatively the response of a real structure or footing is measured. 
2. A rational filter is fitted to the target results, ensuring that nonphysical resonance is avoided. The order of the filter should by high enough to provide a good fit, but low enough to avoid wiggling.

3. Discrete-element models with few internal degrees of freedom are established based on the rational-filter approximation.

This procedure is carried out for each degree of freedom and the discrete-element models are then assembled with a finite-element, or similar, model of the structure. Typically, lumped-parameter models with a three to four internal degrees of freedom provide results of sufficient accuracy. This has been demonstrated in the present chapter for two different cases, namely a footing on a stratified ground and a flexible skirted foundation in homogeneous soil.

\section{References}

Abramowitz, M. \& Stegun, I. (1972). Handbook of Mathematical Functions with Formulas, Graphs and Mathematical Tables, 10 edn, National Bureau of Standards, United States Department of Commerce.

Achmus, M., Kuo, Y.-S. \& Abdel-Rahman, K. (2009). Behavior of monopile foundations under cyclic lateral load, Computers and Geotechnics 36(5): 725-735. PT: J; NR: 15; TC: 3; J9: COMPUT GEOTECH; PG: 11; GA: 447YE; UT: ISI:000266227200005.

Ahmad, S. \& Rupani, A. (1999). Horizontal impedance of square foundationsi in layered soil, Soil Dynamics and Earthquake Engineering 18: 59-69.

Allotey, N. \& El Naggar, M. H. (2008). Generalized dynamic winkler model for nonlinear soil-structure interaction analysis, Canadian Geotechnical Journal 45(4): 560-573. PT: J; UT: ISI:000255885600008.

Andersen, L. (2002). Wave Propagation in Inifinite Structures and Media, PhD thesis, Department of Civil Engineering, Aalborg University, Denmark.

Andersen, L. (2010). Assessment of lumped-parameter models for rigid footings, Computers E Structures 88: 1333-1347.

Andersen, L. \& Clausen, J. (2008). Impedance of surface footings on layered ground, Computers $\mathcal{E}$ Structures 86: 72-87.

Andersen, L., Ibsen, L. \& Liingaard, M. (2009). Lumped-parameter model of a bucket foundation, in S. Pietruszczak, G. N. Pande, C. Tamagnini \& R. Wan (eds), Computational Geomechanics: COMGEO I, IC2E International Center for Computational Engineering, pp. 731-742.

API (2000). Recommended practice for planning, designing and constructing fixed offshore platforms, Rp2a-wsd, American Petroleum Institute, Dallas, Texas, United States of America.

Asgarian, B., Fiouz, A. \& Talarposhti, A. S. (2008). Incremental Dynamic Analysis Considering Pile-Soil-Structure Interaction for the Jacket Type Offshore Platforms. PT: B; CT: 27th International Conference on Offshore Mechanics and Arctic Engineering; CY: JUN 15-20, 2008; CL: Estoril, PORTUGAL; UT: ISI:000263876000031.

Auersch, L. (1988). Wechselwirkung starrer und flexibler Strukturen mit dem Baugrund inbesondere bei Anregnung durch Bodenerschütterungen, BAM-Forschungsbericht 151, Berlin. 
Auersch, L. (1994). Wave Propagation in Layered Soils: Theoretical Solution in Wavenumber Domain and Experimental Results of Hammer and Railway Traffic Excitation, Journal of Sound and Vibration 173(2): 233-264.

Avilés, J. \& Pérez-Rocha, L. (1996). A simplified procedure for torsional impedance functions of embedded foundations in a soil layer, Computers and Geotechnics 19(2): 97-115.

Bathe, K.-J. (1996). Finite-Element Procedures, 1 edn, John Wiley \& Sons Ltd., Chichester.

Brebbia, C. (1982). Boundary Element Methods in Engineering, Springer, Berlin.

$\mathrm{Bu}, \mathrm{S}$. \& Lin, C. (1999). Coupled horizontal-rocking impedance functions for Embedded Square Foundations at High Frequency Factors, Journal of Earthquake Engineering 3(4): 561-587.

DNV (2001). Foundations, Classification Notes No. 30.4, Det Norske Veritas Classifications A/S, Høvik, Norway.

Domínguez, J. (1993). Boundary Elements in Dynamics, Computational Mechanics Publications, Southampton.

El Naggar, M. \& Bentley, K. J. (2000). Dynamic analysis for laterally loaded piles and dynamic p-y curves, Canadian Geotechnical Journal 37(6): 1166-1183. PT: J; NR: 22; TC: 19; J9: CAN GEOTECH J; PG: 18; GA: 388NU; UT: ISI:000166188300002.

El Naggar, M. \& Novak, M. (1994a). Non-linear model for dynamic axial pile response, Journal of Geotechnical Engineering, ASCE 120(4): 308-329.

El Naggar, M. \& Novak, M. (1994b). Nonlinear axial interaction in pile dynamics, Journal of Geotechnical Engineering, ASCE 120(4): 678-696.

El Naggar, M. \& Novak, M. (1995). Nonlinear lateral interaction in pile dynamics, Soil Dynamics and Earthquake Engineering 14: 141-157.

El Naggar, M. \& Novak, M. (1996). Nonlinear analysis for dynamic lateral pile response, Soil Dynamics and Earthquake Engineering 15: 233-244.

Elleithy, W., Al-Gahtani, H. \& El-Gebeily, M. (2001). Iterative coupling of BE and FE method in elastostatics, Engineering Analysis with Boudnary Elements 25: 685-695.

Emperador, J. \& Domínguez, J. (1989). Dynamic response of axisymmetric embedded foundations, Earthquake Engineering and Structural Dynamics 18: 1105-1117.

Gerolymos, N. \& Gazetas, G. (2006a). Development of winkler model for static and dynamic response of caisson foundations with soil and interface nonlinearities, Soil Dynamics and Earthquake Engineering 26(5): 363-376. PT: J; NR: 31; TC: 5; J9: SOIL DYNAM EARTHQUAKE ENG; PG: 14; GA: 032QW; UT: ISI:000236790500003.

Gerolymos, N. \& Gazetas, G. (2006b). Static and dynamic response of massive caisson foundations with soil and interface nonlinearities-validation and results, Soil Dynamics and Earthquake Engineering 26(5): 377-394. PT: J; NR: 31; TC: 5; J9: SOIL DYNAM EARTHQUAKE ENG; PG: 14; GA: 032QW; UT: ISI:000236790500003.

Gerolymos, N. \& Gazetas, G. (2006c). Winkler model for lateral response of rigid caisson foundations in linear soil, Soil Dynamics and Earthquake Engineering 26(5): 347-361. PT: J; NR: 31; TC: 5; J9: SOIL DYNAM EARTHQUAKE ENG; PG: 14; GA: 032QW; UT: ISI:000236790500003.

Haskell, N. (1953). The Dispersion of Surface Waves on Multilayered Medium, Bulletin of the Seismological Society of America 73: 17-43.

Houlsby, G. T., Kelly, R. B., Huxtable, J. \& Byrne, B. W. (2005). Field trials of suction caissons in clay for offshore wind turbine foundations, Géotechnique 55(4): 287-296.

Houlsby, G. T., Kelly, R. B., Huxtable, J. \& Byrne, B. W. (2006). Field trials of suction caissons in sand for offshore wind turbine foundations, Géotechnique 56(1): 3-10. 
Ibsen, L. (2008). Implementation of a new foundations concept for offshore wind farms, Proceedings of the 15th Nordic Geotechnical Meeting, Sandefjord, Norway, pp. 19-33.

Jones, C., Thompson, D. \& Petyt, M. (1999). TEA - a suite of computer programs for elastodynamic analysis using coupled boundary elements and finite elements, ISVR Technical Memorandum 840, Institute of Sound and Vibration Research, University of Southampton.

Jonkman, J. \& Buhl, M. (2005). Fast user's guide, Technical Report NREL/EL-500-38230, National Renewable Energy Laboratory, Colorado, United States of America.

Kong, D., Luan, M., Ling, X. \& Qiu, Q. (2006). A simplified computational method of lateral dynamic impedance of single pile considering the effect of separation between pile and soils, in M. Luan, K. Zen, G. Chen, T. Nian \& K. Kasama (eds), Recent development of geotechnical and geo-environmental engineering in Asia, p. 138. PT: B; CT: 4th Asian Joint Symposium on Geotechnical and Geo-Environmental Engineering (JS-Dalian 2006); CY: NOV 23-25, 2006; CL: Dalian, PEOPLES R CHINA; UT: ISI:000279628900023.

Krenk, S. \& Schmidt, H. (1981). Vibration of an Elastic Circular Plate on an Elastic Half Space-A Direct Approach, Journal of Applied Mechanics 48: 161-168.

Larsen, T. \& Hansen, A. (2004). Aeroelastic effects of large blade deflections for wind turbines, in D. U. of Technology (ed.), The Science of Making Torque from Wind, Roskilde, Denmark, pp. 238-246.

Liingaard, M. (2006). DCE Thesis 3: Dynamic Behaviour of Suction Caissons, PhD thesis, Department of Civil Engineering, Aalborg University, Denmark.

Liingaard, M., Andersen, L. \& Ibsen, L. (2007). Impedance of flexible suction caissons, Earthquake Engineering and Structural Dynamics 36(13): 2249-2271.

Liingaard, M., Ibsen, L. B. \& Andersen, L. (2005). Vertical impedance for stiff and flexible embedded foundations, 2nd International symposium on Environmental Vibrations: Prediction, Monitoring, Mitigation and Evaluation (ISEV2005), Okayama, Japan.

Luco, J. (1976). Vibrations of a Rigid Disk on a Layered Viscoelastic Medium, Nuclear Engineering and Design 36(3): 325-240.

Luco, J. \& Westmann, R. (1971). Dynamic Response of Circular Footings, Journal of Engineering Mechanics, ASCE 97(5): 1381-1395.

Manna, B. \& Baidya, D. K. (2010). Dynamic nonlinear response of pile foundations under vertical vibration-theory versus experiment, Soil Dynamics and Earthquake Engineering 30(6): 456-469. PT: J; UT: ISI:000276257800004.

Mita, A. \& Luco, J. (1989). Impedance functions and input motions for embedded square foundations, Journal of Geotechnical Engineering, ASCE 115(4): 491-503.

Mustoe, G. (1980). A combination of the finite element and boundary integral procedures, $\mathrm{PhD}$ thesis, Swansea University, United Kingdom.

Newmark, N. (1959). A method of computation for structural dynamics, ASCE Journal of the Engineering Mechanics Division 85(EM3): 67-94.

Novak, M. \& Sachs, K. (1973). Torsional and coupled vibrations of embedded footings, Earthquake Engineering and Structural Dynamics 2: 11-33.

Petyt, M. (1998). Introduction to Finite Element Vibration Analysis, Cambridge: Cambridge University Press.

Senders, M. (2005). Tripods with suction caissons as foundations for offshore wind turbines on sand, Proceedings of International Symposium on Frontiers in Offshore Geotechnics: ISFOG 2005, Taylor \& Francis Group, London, Perth, Australia. 
Sheng, X., Jones, C. \& Petyt, M. (1999). Ground Vibration Generated by a Harmonic Load Acting on a Railway Track, Journal of Sound and Vibration 225(1): 3-28.

Stamos, A. \& Beskos, D. (1995). Dynamic analysis of large 3-d underground structures by the BEM, Earthquake Engineering and Structural Dynamics 24: 917-934.

Thomson, W. (1950). Transmission of Elastic Waves Through a Stratified Solid Medium, Journal of Applied Physics 21: 89-93.

Varun, Assimaki, D. \& Gazetas, G. (2009). A simplified model for lateral response of large diameter caisson foundations-linear elastic formulation, Soil Dynamics and Earthquake Engineering 29(2): 268-291. PT: J; NR: 33; TC: 0; J9: SOIL DYNAM EARTHQUAKE ENG; PG: 24; GA: 389ET; UT: ISI:000262074600006.

Veletsos, A. \& Damodaran Nair, V. (1974). Torsional vibration of viscoelastic foundations, Journal of Geotechnical Engineering Division, ASCE 100: 225-246.

Veletsos, A. \& Wei, Y. (1971). Lateral and rocking vibration of footings, Journal of Soil Mechanics and Foundation Engineering Division, ASCE 97: 1227-1248.

von Estorff, O. \& Kausel, E. (1989). Coupling of boundary and finite elements for soil-structure interaction problems, Earthquake Engineering Structural Dynamics 18: $1065-1075$.

Vrettos, C. (1999). Vertical and Rocking Impedances for Rigid Rectangular Foundations on Soils with Bounded Non-homogeneity, Earthquake Engineering and Structural Dynamics 28: 1525-1540.

Wolf, J. (1991a). Consistent lumped-parameter models for unbounded soil: frequency-independent stiffness, damping and mass matrices, Earthquake Engineering and Structural Dynamics 20: 33-41.

Wolf, J. (1991b). Consistent lumped-parameter models for unbounded soil: physical representation, Earthquake Engineering and Structural Dynamics 20: 11-32.

Wolf, J. (1994). Foundation Vibration Analysis Using Simple Physical Models, Prentice-Hall, Englewood Cliffs, NJ.

Wolf, J. (1997). Spring-dashpot-mass models for foundation vibrations, Earthquake Engineering and Structural Dynamics 26(9): 931-949.

Wolf, J. \& Paronesso, A. (1991). Errata: Consistent lumped-parameter models for unbounded soil, Earthquake Engineering and Structural Dynamics 20: 597-599.

Wolf, J. \& Paronesso, A. (1992). Lumped-parameter model for a rigid cylindrical foundation in a soil layer on rigid rock, Earthquake Engineering and Structural Dynamics 21: 1021-1038.

Wong, H. \& Luco, J. (1985). Tables of impedance functions for square foundations on layered media, Soil Dynamics and Earthquake Engineering 4(2): 64-81.

Wu, W.-H. \& Lee, W.-H. (2002). Systematic lumped-parameter models for foundations based on polynomial-fraction approximation, Earthquake Engineering \& Structural Dynamics 31(7): 1383-1412.

Wu, W.-H. \& Lee, W.-H. (2004). Nested lumped-parameter models for foundation vibrations, Earthquake Engineering \& Structural Dynamics 33(9): 1051-1058.

Øye, S. (1996). FLEX4 - simulation of wind turbine dynamics, in B. Pedersen (ed.), State of the Art of Aeroelastic Codes for Wind Turbine Calculations, Lyngby, Denmark, pp. 71-76.

Yong, Y., Zhang, R. \& Yu, J. (1997). Motion of Foundation on a Layered Soil Medium-I. Impedance Characteristics, Soil Dynamics and Earthquake Engineering 16: 295-306. 


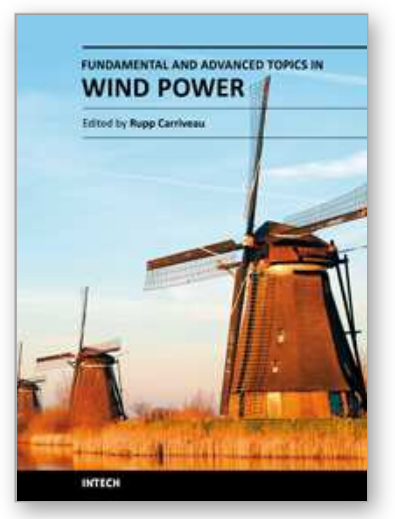

\author{
Fundamental and Advanced Topics in Wind Power \\ Edited by Dr. Rupp Carriveau
}

ISBN 978-953-307-508-2

Hard cover, 422 pages

Publisher InTech

Published online 20, June, 2011

Published in print edition June, 2011

As the fastest growing source of energy in the world, wind has a very important role to play in the global energy mix. This text covers a spectrum of leading edge topics critical to the rapidly evolving wind power industry. The reader is introduced to the fundamentals of wind energy aerodynamics; then essential structural, mechanical, and electrical subjects are discussed. The book is composed of three sections that include the Aerodynamics and Environmental Loading of Wind Turbines, Structural and Electromechanical Elements of Wind Power Conversion, and Wind Turbine Control and System Integration. In addition to the fundamental rudiments illustrated, the reader will be exposed to specialized applied and advanced topics including magnetic suspension bearing systems, structural health monitoring, and the optimized integration of wind power into micro and smart grids.

\title{
How to reference
}

In order to correctly reference this scholarly work, feel free to copy and paste the following:

Lars Andersen and Johan Clausen (2011). Efficient Modelling of Wind Turbine Foundations, Fundamental and Advanced Topics in Wind Power, Dr. Rupp Carriveau (Ed.), ISBN: 978-953-307-508-2, InTech, Available from: http://www.intechopen.com/books/fundamental-and-advanced-topics-in-wind-power/efficient-modelling-ofwind-turbine-foundations

\section{INTECH}

open science | open minds

\author{
InTech Europe \\ University Campus STeP Ri \\ Slavka Krautzeka 83/A \\ 51000 Rijeka, Croatia \\ Phone: +385 (51) 770447 \\ Fax: +385 (51) 686166 \\ www.intechopen.com
}

\author{
InTech China \\ Unit 405, Office Block, Hotel Equatorial Shanghai \\ No.65, Yan An Road (West), Shanghai, 200040, China \\ 中国上海市延安西路65号上海国际贵都大饭店办公楼 405 单元 \\ Phone: +86-21-62489820 \\ Fax: +86-21-62489821
}


(C) 2011 The Author(s). Licensee IntechOpen. This chapter is distributed under the terms of the Creative Commons Attribution-NonCommercialShareAlike-3.0 License, which permits use, distribution and reproduction for non-commercial purposes, provided the original is properly cited and derivative works building on this content are distributed under the same license. 